Aus der Abteilung Anaesthesiologie

(Prof. Dr. med. M. Quintel)

im Zentrum Anaesthesiologie-, Rettungs- und Intensivmedizin

der Medizinischen Fakultät der Universität Göttingen

\title{
Auswirkungen einer akuten, intrakraniellen Druckerhöhung auf die computertomographisch bestimmte Lungenparenchymdichte und das extravaskuläre Lungenwasser in gesunden und geschädigten Schweinelungen
}

\author{
INAUGURAL - DISSERTATION \\ zur Erlangung des Doktorgrades \\ der medizinischen Fakultät der \\ Georg-August-Universität zu Göttingen
}

vorgelegt von

Philip Sauter

aus

$\underline{\text { Gelsenkirchen }}$ 
I. Berichterstatter: Prof. Dr. med. Michael Quintel II. Berichterstatter: Prof. Dr. med. Veit Rohde

III. Berichterstatter: Prof. Dr. med. Joachim Lotz 


\section{Inhaltsverzeichnis}

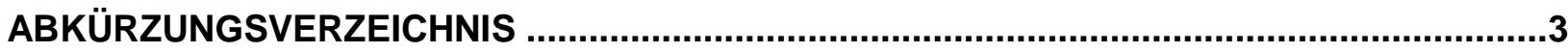

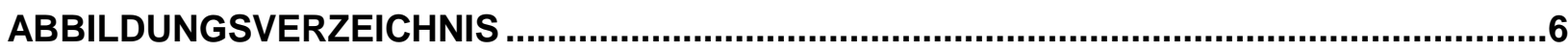

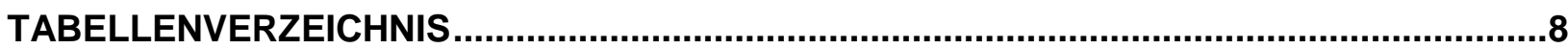

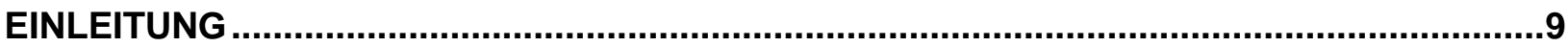

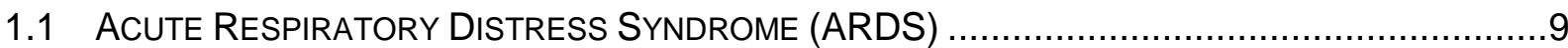

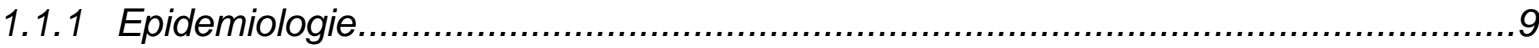

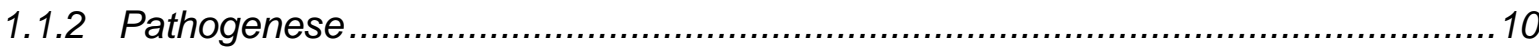

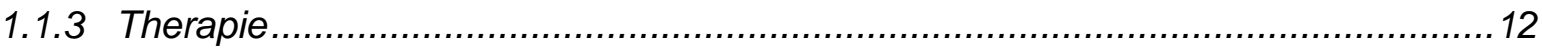

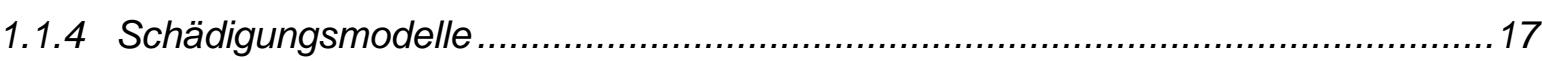

1.2 SCHÄDEL-HIRN-TRAUMA, ACUTE INTRACRANIAL HYPERTENSION (AICH) .........................17

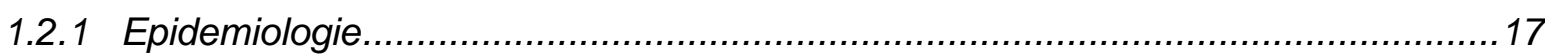

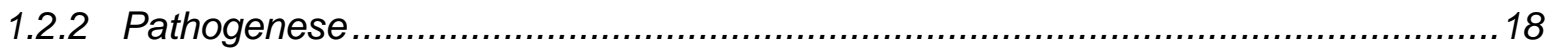

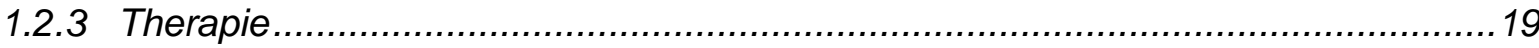

1.3 SCHÄDEL-HIRN-TRAUMA UND ACUTE ReSPIRATORY DISTRESS SYNDROME ......................20

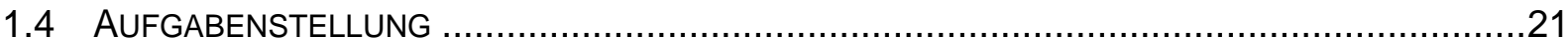

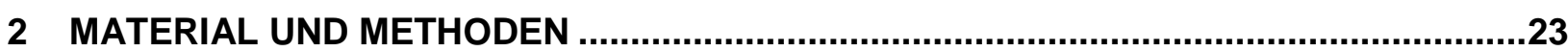

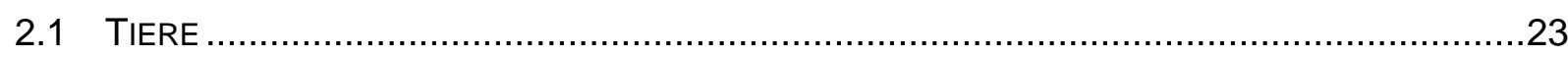

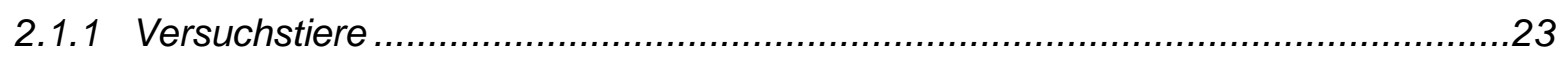

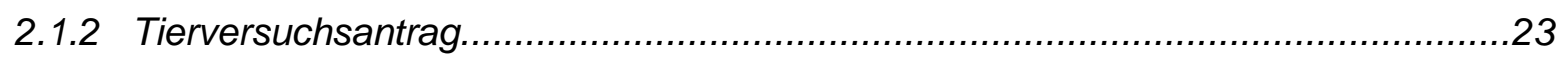

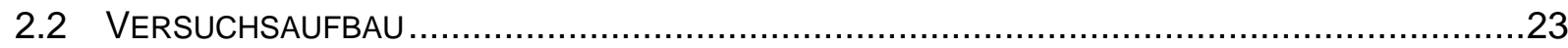

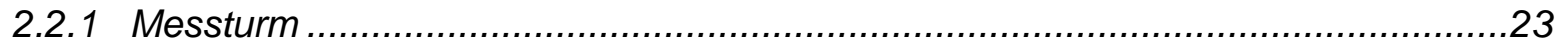

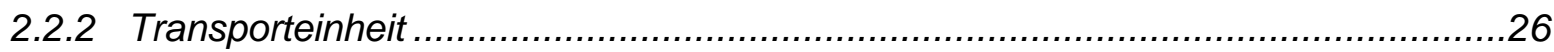

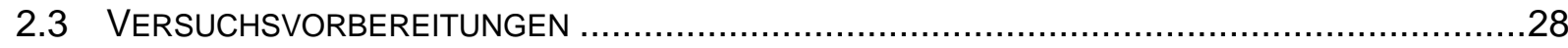

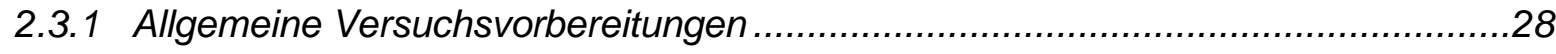

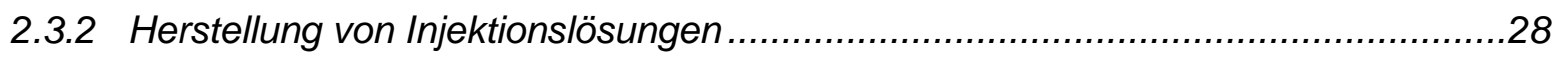

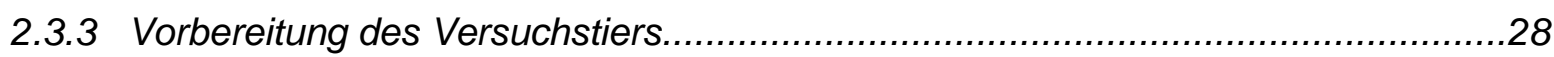

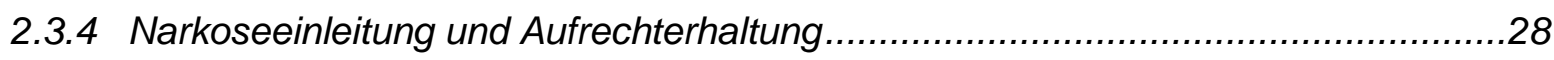

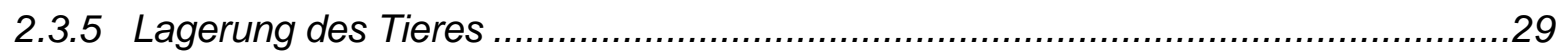

2.3.6 Messung von Hämodynamik und Gasaustausch ...............................................30

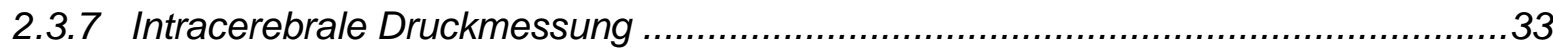

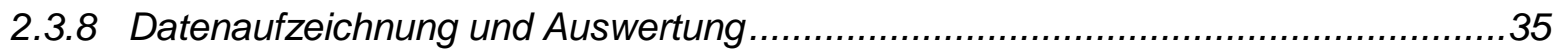

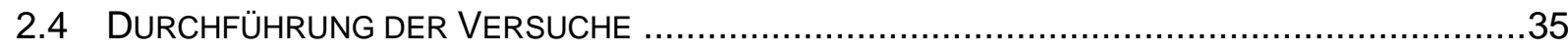

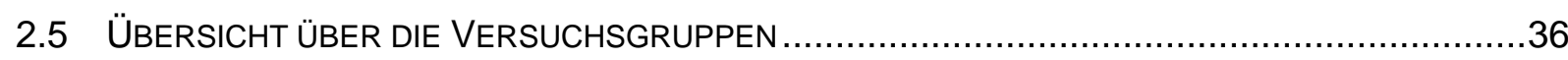

2.5.1 Gruppe 1 - Kontrollgruppe (Control) …….................................................. 


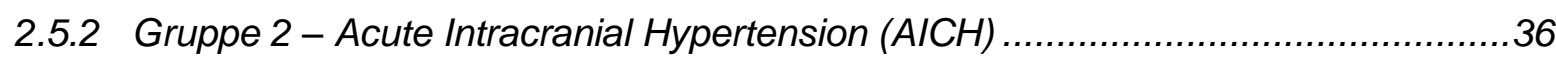

2.5.3 Gruppe 3-Acute Respiratory Distress Syndrome (ARDS) ................................37

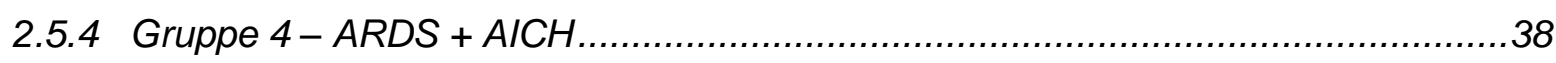

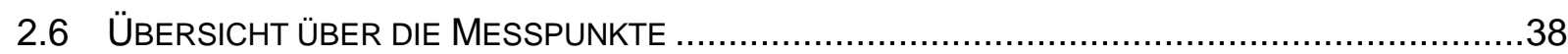

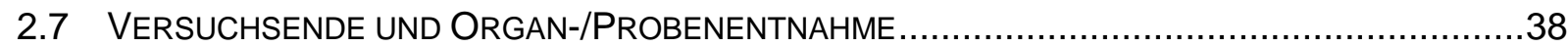

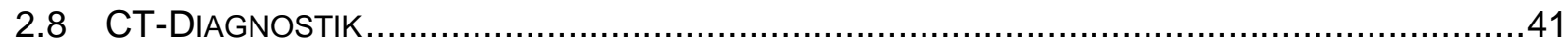

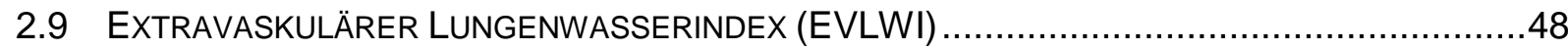

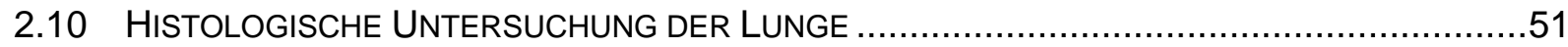

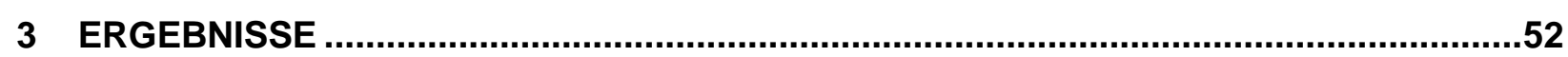

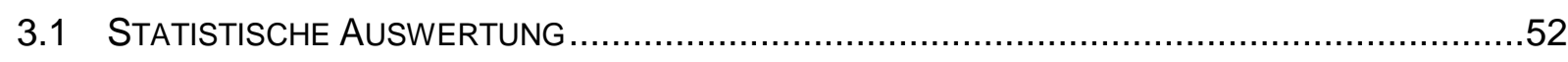

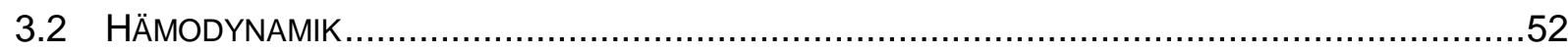

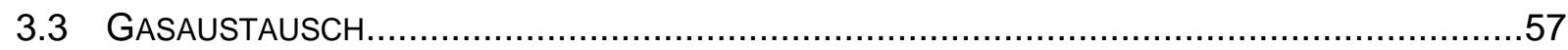

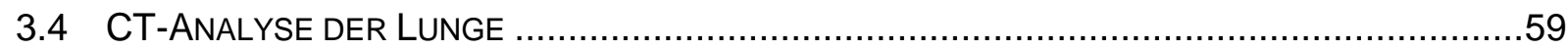

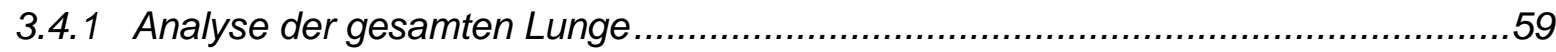

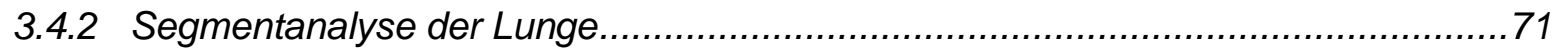

3.5 EXTRAVASKULÄRER LUNGENWASSERINDEX UND HOUNSFIELD UNITS ............................80

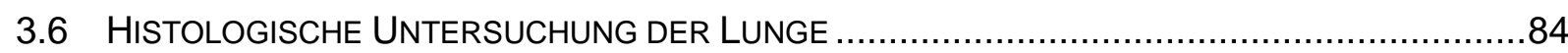

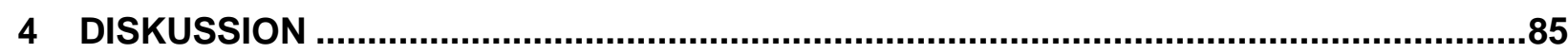

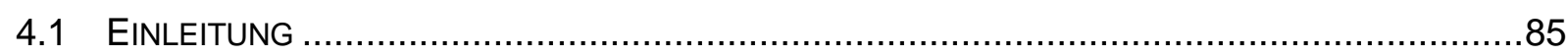

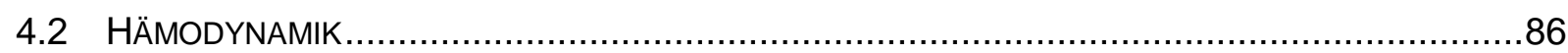

4.2.1 Blutdruck, Herzfrequenz, Schlagvolumen, Herzzeitvolumen................................86

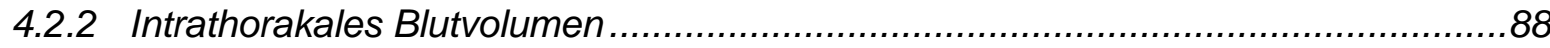

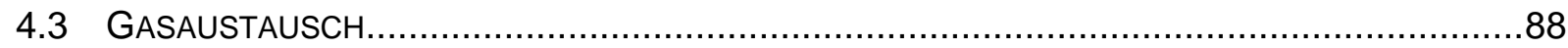

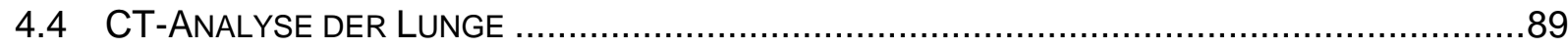

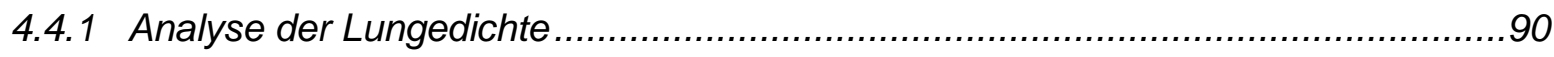

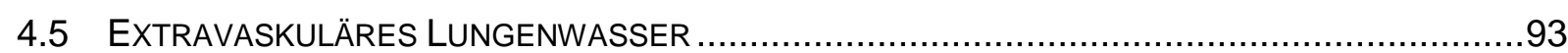

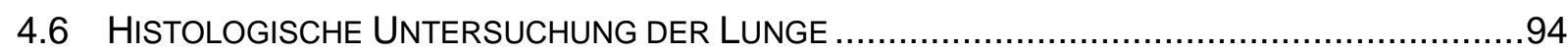

4.7 EINE KRITISCHE BETRACHTUNG DES VERSUCHSAUfBAUS .......................................94

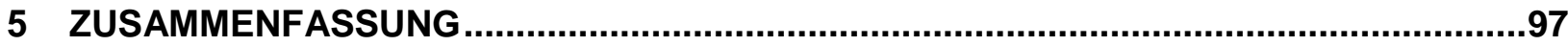

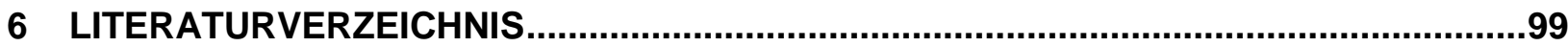




\section{Abkürzungsverzeichnis}

$\mathrm{AICH} \quad$ acute intracranial hypertension, akuter intrakranieller Hochdruck

ALI acute lung injury, akuter Lungenschaden

ARDS acute respiratory distress syndrome, akutes Lungenversagen

art. arteriell, $-e,-r$

bzw. beziehungsweise

CCT kraniale Computertomographie

$\mathrm{CO}_{2} \quad$ Kohlenstoffdioxid

CPP cerebral perfusion pressure, cerebraler Perfusionsdruck

CT Computertomograph(ie)

DICOM Digital Imaging and Communication in Medicine

DSt downslope time

ECMO extrakorporale Membranoxygenierung

EKG Elektrokardiogramm

et al. und andere

etc. et cetera

EVLW extravascular lung water, extravaskuläres Lungenwasser

EVLWI extravascular lung water index, extravaskulärer Lungenwasserindex

$\mathrm{FiO}_{2}$ inspiratorische Sauerstoffkonzentration

g Gramm

GCS Glasgow Coma Scale

GEDV global enddiastolic volume, globales enddiastolisches Volumen

HF Herzfrequenz

HFJV high frequency jet ventilation

HFOV high frequency oscillatory ventilation

HFPPV high frequency positive pressure ventilation

HU Hounsfield Units, Hounsfield-Einheiten

$\mathrm{HZV}$ Herzzeitvolumen

ICP intracranial pressure, intrakranieller Druck

i.e. id est, „das ist“, „mit anderen Worten”

IL-1B Interleukin 1 Beta

IL-6 Interleukin 6

ITBI Intrathorakaler Blutvolumenindex 


$\begin{array}{ll}\text { ITBV } & \text { Intrathorakales Blutvolumen } \\ \text { ITTV } & \text { Intrathorakales Thermovolumen } \\ \text { i.v. } & \text { intravenös } \\ \text { KG } & \text { Körpergewicht } \\ \text { kg } & \text { Kilogramm } \\ \text { kV } & \text { Kilovolt } \\ \text { LAEDV } & \text { linksatriales enddiastolisches Volumen } \\ \text { LVEDV } & \text { linksventrikuläres enddiastolisches Volumen } \\ \text { m }^{2} & \text { Quadratmeter } \\ \text { MA } & \text { Megaampere } \\ \text { MALUNA } & \text { Mannheimer Lungen Analyse Tool } \\ \text { MAP } & \text { mean arterial pressure, mittlerer arterieller Druck } \\ \text { mg } & \text { Milligramm } \\ \text { min } & \text { Minute(n) } \\ \text { ml } & \text { Milliliter } \\ \text { mmHg } & \text { Millimeter Quecksilbersäule } \\ \text { MOF } & \text { multi organ failure, Multiorganversagen } \\ \text { MPAP } & \text { mean pulmonary arterial pressure, mittlerer pulmonalarterieller Druck } \\ \text { MTt } & \text { mean transit time } \\ \text { Mg } & \text { Mikrogramm } \\ \text { NPÖ } & \text { neurogenes pulmonales Ödem } \\ \text { PaCO } & \text { arterieller Kohlendioxidpartialdruck } \\ \text { PaO } & \text { arterieller Sauerstoffpartialdruck } \\ \text { PCWP } & \text { pulmonary capillary wedge pressure, pulmonalarterieller Verschlussdruck } \\ \text { PEEP } & \text { positive end-expiratory pressure, positiver endexspiratorischer Druck } \\ \text { PH } & \text { pulmonale Hypertonie } \\ \text { pH } & \text { Wasserstoffionenkonzentration } \\ \text { PICCO } & \text { Puls Contour Cardiac Output } \\ \text { PTV } & \text { pulmonary thermovolume, pulmonales Thermovolumen } \\ \text { RAEDV } & \text { rechtsatriales enddiastolisches Volumen } \\ \text { ROI } & \text { region of interest } \\ \text { RVEDV } & \text { rechtsventrikuläres enddiastolisches Volumen } \\ \text { sec. } & \text { Sekunde(n) } \\ \text { SHT } & \text { Schädel-Hirn-Trauma } \\ \end{array}$


TBI traumatic brain injury, traumatischer Hirnschaden

TNF-alpha Tumor-Nekrose-Faktor alpha

u.a. unter anderem

v.a.m. vieles Andere mehr

V/Q Ventilations/Perfusions-Verhältnis

VALI ventilator associated lung injury, beatmungsassoziierte Lungenschädigung

VILI ventilator induced lung injury, beatmungsbedingte Lungenschädigung

vs. gegen

Vt Tidalvolumen

ZVD zentralvenöser Druck 


\section{Abbildungsverzeichnis}

Abbildung 1: Normales Ventilations-Perfusions- (V/Q) Verhältnis ...............................11

Abbildung 2: Ventilatons-Perfusions-Verhältnis bei ARDS mit Rechts-Links-Shunt ......11

Abbildung 3: Schematische Darstellung des Messturms...........................................24

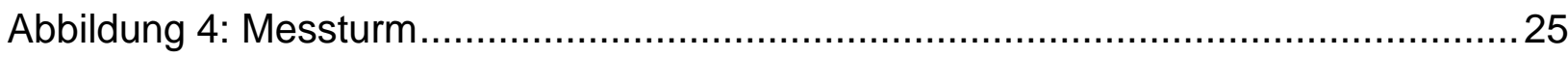

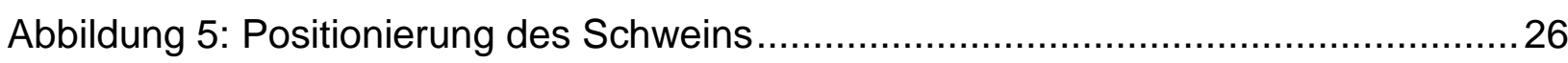

Abbildung 6: Schematische Darstellung der Transporteinheit..................................27

Abbildung 7: Versuchstier mit Messturm und Transporteinheit ..................................27

Abbildung 8: Lagerung im Tier-OP 2 ............................................................... 30

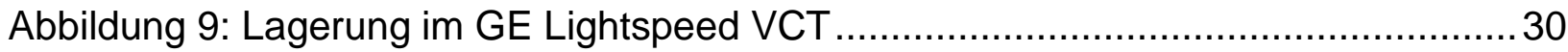

Abbildung 10: Datenverarbeitung der Hämodynamik ............................................... 31

Abbildung 11: Ultraschallgesteuerte Anlage eines PICCO-Katheters ..........................32

Abbildung 12: Datenverarbeitung intracerebrale Druckmessung ……........................33

Abbildung 13: Platzierung der intrakraniellen Sonden mittels Bohrlochtrepanation........34

Abbildung 14: links - Bohrer, Sonde, Katheter; rechts - Darstellung im CCT ................37

Abbildung 15: links - gesunde Lunge $\left(T_{0}\right)$; rechts - Lunge nach Schädigung durch

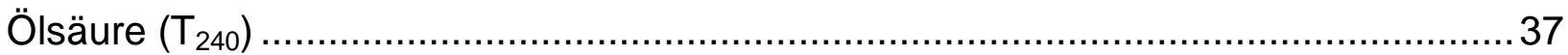

Abbildung 16: Längsschnittthorakotomie und eröffnetes Perikard................................39

Abbildung 17: Tabaksbeutelnaht und Perfusionskanüle im rechten Vorhof ................... 39

Abbildung 18: Inzision der Aorta und Platzierung der Perfusionskanüle .......................40

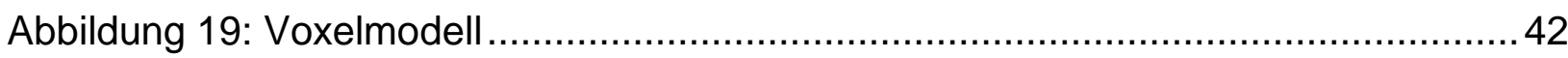

Abbildung 20: Lungenkompartimente nach Hounsfield Units eingeteilt..........................44

Abbildung 21: Schematische Segmenteinteilung der Lunge vor Schädigung ...............46

Abbildung 22: Schematische Segmenteinteilung der Lunge nach Schädigung ..............46

Abbildung 23: Beispiel - CT-Bilder der Lunge zum Zeitpunkt T240 …....................... 47

Abbildung 24: Thermodilutionsmethode - HZV (Pulsion 2002) ................................. 49

Abbildung 25: Thermodilution - Volumenparameter (Pulsion 2002) .............................49

Abbildung 26: Entwicklung der Herzfrequenz in allen Gruppen................................54

Abbildung 27: Entwicklung des Schlagvolumens in allen Gruppen ............................55

Abbildung 28: Entwicklung des Herzzeitvolumens in allen Gruppen ...........................56

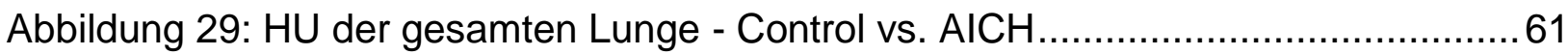

Abbildung 30: $\mathrm{HU}$ der gesamten Lunge - ARDS vs. ARDS + AICH .............................62

Abbildung 31: Gasgehalt der gesamten Lunge - ml/g - Control vs. AlCH.....................63

Abbildung 32: Gasgehalt der gesamten Lunge - ml/g - ARDS vs. ARDS + AICH .........64 
Abbildung 33: Normal ventilierte Lunge - Control vs. AICH 65

Abbildung 34: Normal ventilierte Lunge - ARDS vs. ARDS + AICH . 66

Abbildung 35: Schlecht ventilierte Lunge - CG vs. AICH .......................................67

Abbildung 36: Schlecht ventilierte Lunge - ARDS vs. ARDS + AICH ........................68

Abbildung 37: Nicht ventilierte Lunge - Control vs. AICH .....................................69

Abbildung 38: Nicht ventilierte Lunge - ARDS vs. ARDS + AICH ........................... 70

Abbildung 39: Delta HU von $\mathrm{T}_{240}-\mathrm{T}_{0}$ - Control vs. AICH ...................................... 78

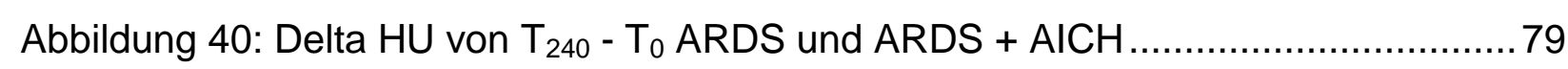

Abbildung 41: Extravaskulärer Lungenwasserindex (EVLWI) $-\mathrm{T}_{0}-\mathrm{T}_{240} \ldots \ldots \ldots \ldots \ldots \ldots . . . . . . . .11$

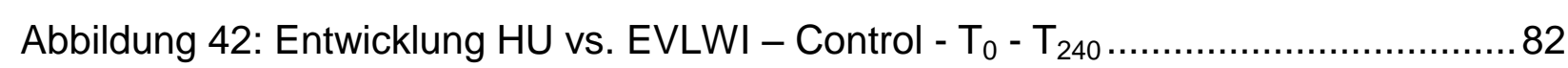

Abbildung 43: Entwicklung HU vs. EVLWI - AICH - $\mathrm{T}_{0}-\mathrm{T}_{240 \ldots \ldots \ldots \ldots \ldots \ldots \ldots \ldots \ldots \ldots \ldots \ldots \ldots \ldots \ldots \ldots \ldots \ldots \ldots \ldots \ldots \ldots \ldots}$

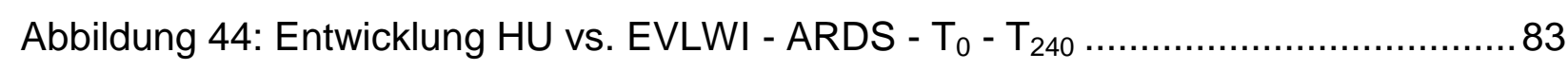

Abbildung 45: Entwicklung HU vs. EVLWI - ARDS +AICH - $\mathrm{T}_{0}-\mathrm{T}_{240} \ldots \ldots \ldots \ldots \ldots \ldots \ldots \ldots . . . \ldots 3$ 


\section{Tabellenverzeichnis}

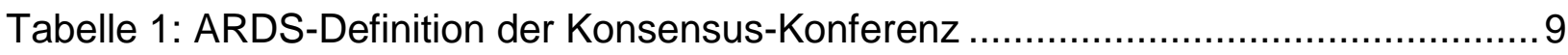

Tabelle 2: Einteilung des Schweregrades eines Schädel-Hirn-Traumas.......................19

Tabelle 3: Übersicht über die Versuchsgruppen........................................................ 36

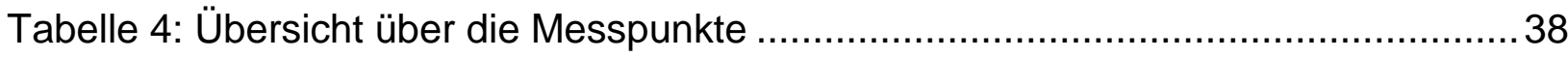

Tabelle 5: Hounsfield-Einteilung verschiedener Lungenbereiche nach Gattinoni .......... 45

Tabelle 6: Histologisches Scoringsystem der Lunge ............................................51

Tabelle 7: Hämodynamik, gemessene und errechnete Parameter...............................53

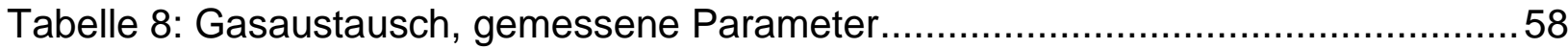

Tabelle 9: CT-Daten der gesamten Lunge $T_{240}-T_{0}$ (Seg. 1 - 10) ................................... 60

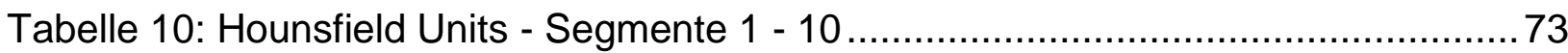

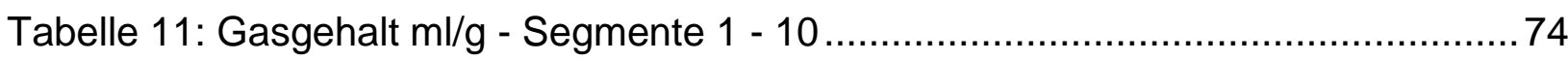

Tabelle 12: Normal ventilierte Lungenareale \% - Segmente 1 - $10 \ldots \ldots \ldots \ldots \ldots \ldots \ldots \ldots \ldots \ldots . . . . .75$

Tabelle 13: Schlecht ventilierte Lungenareale $\%$ - Segmente 1 - $10 \ldots \ldots \ldots \ldots \ldots \ldots \ldots \ldots \ldots \ldots . . . .76$

Tabelle 14: Nicht ventilierte Lungenareale \% - Segmente 1 - 10 ..................................77

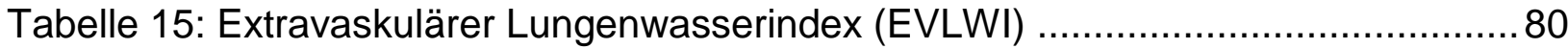

Tabelle 16: Histopathologisches Scoring der Lungenschädigung ............................... 84 


\section{Einleitung}

\subsection{Acute Respiratory Distress Syndrome (ARDS)}

\subsubsection{Epidemiologie}

Der Begriff ARDS wurde erstmalig von Ashbaugh et al. im Jahre 1967 genannt (Ashbaugh et al. 1967). Er beschreibt ein Syndrom, welches sich aus charakteristischen klinischen Symptomen und pathophysiologischen Veränderungen zusammensetzt und somit den Eindruck eines uniformen Krankheitsgeschehens vermittelt. Zu den klinischen Symptomen gehören Dyspnoe, Tachypnoe und eine Hypoxämie. In der Bildgebung der Lunge sieht man diffuse, bilaterale, alveoläre Infiltrate. Pathophysiologisch kommt es hier zu einem vergrößerten intrapulmonalen Rechts-Links-Shunt, einem Lungenödem, einer pulmonalarteriellen Hypertonie $(\mathrm{PH})$ und einer verminderten pulmonalen Compliance (Neumann und Hedenstierna 2001). Verschiedene Kriterien zur Diagnostik eines Acute Respiratory Distress Syndrome wurden von der Amerikanisch-Europäischen Konsensus-Konferenz festgesetzt (Bernard et al. 1994). Hierbei wurde zwischen Acute Lung Injury (ALI) und Acute Respiratory Distress Syndrome (ARDS) unterschieden. Im deutschsprachigen Raum werden die Begriffe ALI und akute Lungenschädigung sowie ARDS und akutes Lungenversagen häufig synonym verwandt.

Tabelle 1: ARDS-Definition der Konsensus-Konferenz

ALI

ARDS

\begin{tabular}{lll}
\hline Zeitlicher Verlauf & akut & akut \\
paO2/FiO2 $(\mathbf{1 , 0})$ (peep unabh.) & $<300 \mathrm{mmHg}$ & $<200 \mathrm{mmHg}$ \\
Thoraxröntgenbild & bilaterale Infiltrate & bilaterale Infiltrate \\
PCWP & $<18 \mathrm{mmHg}$ & $<18 \mathrm{mmHg}$
\end{tabular}

oder kein klinischer Hinweis auf linksatriale Hypertonie

In der obigen Tabelle ist die Einteilung/Definition einer akuten Lungenschädigung (ALI) bzw. eines akuten Lungenversagens (ARDS) nach den Kriterien der Konsensus-Konferenz dargestellt (Bernard et al. 1994).

Weltweit wird die Inzidenz mit ca. 3 - 88 Fällen pro 100.000 Einwohnern angegeben (Arroliga et al. 2002, Atabai und Matthay 2002, Brun-Buisson et al. 2004, Goss et al. 2003, Rubenfeld et al. 2005). Aufgrund dieser hohen Inzidenz und seiner mit $40-60 \%$ 
hohen Letalität (Rubenfeld et al. 2005) stellt dieses Krankheitsbild große Anforderungen an die intensivmedizinische Betreuung.

In $75 \%$ aller Fälle wird das Acute Respiratory Distress Syndrome durch eine der folgenden vier Ätiologien hervorgerufen: Polytrauma, Pneumonie, Sepsis oder Aspiration. Des Weiteren unterscheidet man ein ARDS nach pulmonaler, direkter Lungenschädigung und nach extrapulmonaler, indirekter Schädigung. Zu den direkten Schädigungen gehören z.B. pulmonale Infektionen, Aspiration von Mageninhalt, Fettembolie, BeinaheErtrinken und Lungenkontusion. Die indirekte Schädigung ist z.B. auf Sepsis, Polytrauma, Massivtransfusionen oder Verbrennungen zurückzuführen (Arroliga et al. 2002, Bernard et al. 1994).

Die hohe Mortalität ist nicht in erster Linie Folge der respiratorischen Kompromittierung, sondern vielmehr durch das Multiorganversagen (MOF = Multi Organ Failure) in Folge eines septischen Schocks bedingt (Bell et al. 1983, Montgomery et al. 1985). Vergleichsstudien haben gezeigt, dass innerhalb der letzten zwei Jahrzehnte eine Reduzierung der Mortalität aufgrund verbesserter Therapiestrategien möglich war (Abel et al. 1998, Jardin et al. 1999, Milberg et al. 1995, The Acute Respiratory Distress Syndrome Network 2000). Ein effektiver und zeitnaher Behandlungsbeginn sowie verbesserte Beatmungstechniken stehen hierbei im Vordergrund (Brower et al. 2001).

\subsubsection{Pathogenese}

Hauptursächlich für die Entstehung eines ARDS ist die Entwicklung eines massiven pulmonalen Ödems mit einer Diffusionsstörung sowohl für Sauerstoff als auch für Kohlendioxid. Eine starke Entzündungsreaktion, hervorgerufen durch eine Vielzahl von Mediatoren (z.B. Prostaglandine, Leukotriene, Faktoren des Gerinnungs- und Komplementsystems, Interleukine, Tumor-Nekrose-Faktor) und Entzündungszellen (Puneet et al. 2005, Strieter und Kunkel 1994, Witko-Sarsat et al. 2000) bewirken eine massive entzündliche Veränderung in der Lunge. Die auslösenden Noxen können, wie bereits beschrieben, vielfältig sein, münden jedoch in dieselben pathologischen und histologischen Veränderungen. Verschiedene ätiologische Faktoren (pulmonal wie extrapulmonal) führen also stets, vermittelt über eine massive Entzündungsreaktion, im Verlauf zu einem ARDS.

Kennzeichen dieser Entzündungsreaktion ist vor allem eine Schädigung der alveolokapillären Membran mit folgender Permeabilitätserhöhung der alveolär-kapillären Barriere. 
Es kommt zum Übertreten von Flüssigkeit, Zellen und Protein in die Alveolen sowie in das pulmonale Interstitium. Die Oberflächenspannung in den Alveolen nimmt durch den Funktionsverlust des Surfactants (Baker et al. 1999) und den Verlust von Surfactant bildenden Pneumozyten des Typs II ab. Dies führt zum Kollaps der Alveolen und zur Bildung von Atelektasen. Diese atelektatischen Lungenareale nehmen nicht mehr am Gasaustausch teil, werden jedoch noch perfundiert. Somit entsteht eine VentilationsPerfusions-Störung und folglich eine Vergrößerung des intrapulmonalen Rechts-LinksShunts (siehe Abbildungen 1 und 2). Das Verteilungsmuster dieser Schädigung innerhalb der Lunge ist sehr inhomogen. Computertomographische Untersuchungen von ARDS-Lungen durch Gattinoni et al. konnten dies eindrücklich nachweisen (Gattinoni et al. 1994). Die Lungenareale wurden demnach in gesunde Bereiche, noch rekrutierbare Bereiche und Bereiche, in denen kein Gasaustausch mehr stattfindet, eingeteilt.

Abbildung 1: Normales Ventilations-Perfusions- (V/Q) Verhältnis

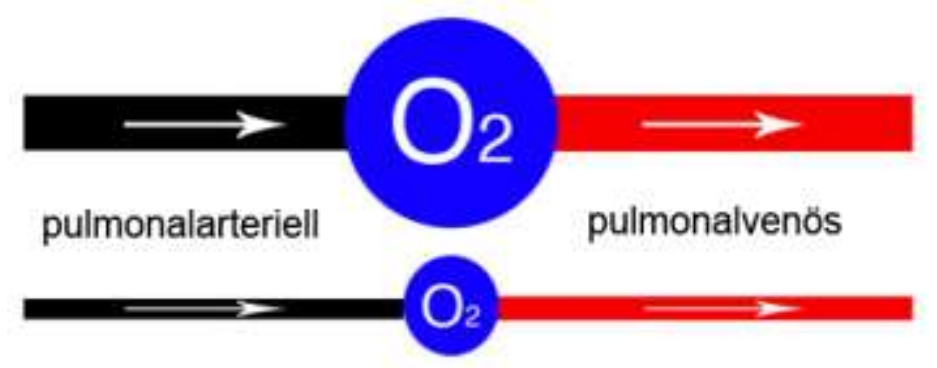

Gut belüftete Lungenareale werden vermehrt perfundiert. Schlecht belüftete Lungenareale werden weniger gut perfundiert.

Abbildung 2: Ventilatons-Perfusions-Verhältnis bei ARDS mit Rechts-Links-Shunt

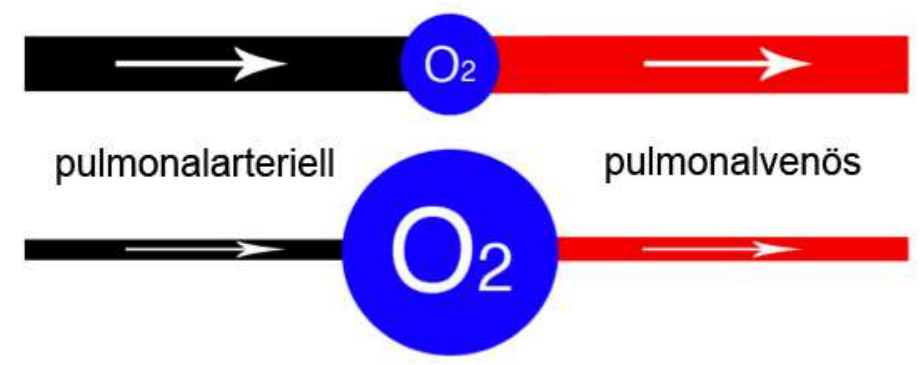

Beim ARDS werden schlecht ventilierte Bereiche noch immer gut perfundiert und normal belüftete Bereiche schlechter durchblutet. 


\subsubsection{Therapie}

Bei der Behandlung des ARDS steht die symptomatische Therapie im Vordergrund; eine kausale Behandlung ist bisher nicht sicher möglich. Lediglich die auslösende Komponente (z.B. Pneumonie, Sepsis) kann kausal therapiert werden, um eine Perpetuierung des Geschehens zu verhindern. Mögliche Komplikationen wie zum Beispiel das Auftreten von nosokomialen Infektionen oder Thrombembolien sollten verhindert werden. Hauptbestandteil der ARDS-Therapie bleibt jedoch die für den Patienten optimale Oxygenierung (Davey-Quinn et al. 1999, Gattinoni et al. 2006) sowie das Verhindern des Fortschreitens der Lungenschädigung.

\subsubsection{Beatmung}

Die zentrale Rolle bei der Behandlung des ARDS spielt die Gasaustauschstörung. Aufgrund des teilweise extrem großen intrapulmonalen Rechts-Links-Shunts, der durch Atelektasen- und Ödembildung entsteht, führt auch die Erhöhung der inspiratorischen Sauerstoffkonzentration $\left(\mathrm{FiO}_{2}\right)$ zu keiner wesentlichen Verbesserung der Oxygenierung. Ziel einer optimalen Beatmung beim akuten Lungenversagen muss daher die Wiederherstellung (Rekrutierung) und Stabilisierung einer ausreichend großen Gasaustauschfläche sein (Becker und Jerrentrup 2001, Gattinoni et al. 2006).

\subsubsection{Lungenprotektive Beatmung}

Zunächst ging man davon aus, die Lungenfunktion von ARDS-Patienten mit Hilfe hoher Atemzugvolumina von $10-15 \mathrm{ml} / \mathrm{kg} \mathrm{KG}$ und geringen endexspiratorischen Drücken (PEEP $=$ Positive End-Expiratory Pressure) zwischen 0-5 $\mathrm{cmH}_{2} \mathrm{O}$ durch Blähen und somit Verhinderung der Bildung und Aufhebung vorhandener Atelektasen zu verbessern (Marini 1996). In tierexperimentellen Studien zeigte sich jedoch bald, dass die Lunge durch ein Baro- und Volutrauma erheblichen Schaden davontrug und sich eine durch Beatmung verursachte Lungenschädigung (VILI = Ventilator Induced Lunge Injury) entwickelte (Dreyfuss und Saumon 1998). Daraufhin wurde die lungenprotektive Beatmung als neues Konzept angewandt. Ziel waren kleine Atemzugvolumina von 6-8 $\mathrm{ml} / \mathrm{kg} \mathrm{KG}$, niedrige Spitzendrücke und ein ausreichend hoher PEEP. Studien konnten zeigen, dass ARDS-Patienten, die mit kleinen Tidalvolumina beatmet wurden, eine bessere Überlebensrate trotz Inkaufnahme eines niedrigeren $\mathrm{PaO}_{2}$ und erhöhten $\mathrm{PaCO}_{2}$ im Rahmen einer permissiven Hyperkapnie und folgender respiratorischer Azidose aufwiesen (Amato et al. 1998, Hickling et al. 1994). Eine Studie des amerikanischen ARDS- 
Netzwerks konnte hierdurch eine Reduktion der Letalität um 22\% von 39,8\% auf 31,9\% nachweisen (The Acute Respiratory Distress Syndrome Network 2000).

Bedingt durch die endotracheale Intubation kommt es zu einem Verlust der natürlichen Glottisfunktion und folglich zu einem starken Absinken der endexspiratorischen Drücke. Um eine vermehrte Atelektasenbildung zu vermeiden bzw. kollabierte Lungenbläschen zu rekrutieren, wird seit etwa vierzig Jahren PEEP eingesetzt (Mclntyre und Laws 1969). Neben einer besseren Oxygenierung durch eine Verminderung des intrapulmonalen Shuntflusses und einer Verbesserung des V/Q-Verhältnisses aufgrund einer erhöhten Residualkapazität kann auch die Entstehung alveolärer Ödeme durch Surfactantverlust reduziert werden. Das Auftreten einer beatmungsassoziierten Lungenschädigung (VALI = Ventilator Associated Lung Injury) durch zyklisches Blähen und nachfolgendes Kollabieren der Alveolen und dadurch auftretende Scherkräfte konnte ebenfalls vermindert werden (Weber-Carstensen und Lohbrunner 1999). Einen negativen Einfluss kann ein hoher PEEP auf die Hämodynamik nehmen. Durch den erhöhten intrathorakalen Druck kommt es zur Veränderung der rechts- sowie linksventrikulären Vor- und Nachlast und somit zu Störungen der hämodynamischen Situation (Pinsky 1990). Die Wahl des optimalen PEEP-Niveaus kann daher nicht pauschal festgesetzt werden, sondern richtet sich individuell nach dem jeweiligen Patienten. Leitwerte in Form von PEEP-FiO ${ }_{2}$-Kombinationen finden sich in einer Studie des ARDS-Netzwerks (The Acute Respiratory Distress Syndrome Network 2000) und können als Richtschnur herangezogen werden. Eine gängige Möglichkeit zur Bestimmung des individuellen PEEP-Niveaus ist der PEEP-Trial. Hierbei werden durch Rekrutierungsmanöver und hohe PEEP-Werte bestmögliche $\mathrm{PaO}_{2} / \mathrm{FiO}_{2}$-Verhältnisse angestrebt, um im Anschluss daran den PEEP schrittweise zu reduzieren und gleichzeitig ein möglichst gutes $\mathrm{PaO}_{2} / \mathrm{FiO}_{2}$-Verhältnis aufrechtzuerhalten (Girgis et al. 2006).

\subsubsection{Open-Lung-Konzept}

Das open-lung-Konzept dient der Rekrutierung atelektatischer Lungenareale und wurde erstmals von Lachmann im Jahre 1992 vorgestellt (Lachmann 1992). Durch eine kurzzeitige Erhöhung des intrapulmonalen Drucks auf ca. $30-60 \mathrm{cmH}_{2} \mathrm{O}$ über einen Zeitraum von etwa 30-60 Sekunden können bereits atelektatische Bereiche eröffnet und durch einen hohen PEEP offen gehalten werden (Engelmann 2000). Meade et al. konnten in ihrer Studie an 983 Patienten mit ARDS einen Vorteil der lungenprotektiven Be- 
atmung mit niedrigen PEEP-Werten und frühzeitigen Rekrutierungsmanövern nachweisen (Meade et al. 2008).

\subsubsection{Hochfrequenzventilation}

Der Begriff Hochfrequenzventilation (HFO) ist definiert durch eine im Vergleich zur Spontanatmung mindestens viermal höhere Atemfrequenz (Biro und Wiedemann 1999) (meistens > 60/min). Man unterteilt dieses Verfahren abhängig von der Impulsfrequenz in: high-frequency positive pressure ventilation (HFPPV), high-frequency jet ventilation (HFJV) und high-frequency oscillatory ventilation (HFOV). Jedes Verfahren hat seine eigenen Vor- und Nachteile und unterscheidet sich durch seine Konstruktion und die Art und Weise der Exspirationsphase. Allen Verfahren ist gemein, dass sie mit sehr kleinen Hubvolumina, meist kleiner als der anatomische Totraum, und niedrigen Spitzendrücken arbeiten und so die mechanische Belastung der Lunge durch eine künstliche, tidale Beatmung weiter reduzieren (Krishnan und Brower 2000, Luecke et al. 2000).

\subsubsection{Extrakorporale Membranoxygenierung}

Die extrakorporale Membranoxygenierung (ECMO) ist eine weitere Ultima Ratio in der Therapie des ARDS. Ihre Anwendung ist spezialisierten Zentren vorbehalten. Mit Hilfe eines Schlauchsystems wird venöses oder arterielles Blut über eine künstliche Lunge decarboxyliert, mit Sauerstoff angereichert und daraufhin wieder dem Gefäßsystem zugeführt. Man unterscheidet hier zwischen arterio-venösen und veno-venösen Systemen. In der Regel kommt dieses Verfahren nur bei schwersten Formen des ARDS zur Anwendung und auch nur dann, wenn bereits alle konservativen Maßnahmen ausgeschöpft wurden (Mols et al. 2000). Ziel dieses Verfahrens ist die Abwendung einer lebensbedrohlichen Hypoxie. Systeme wie z.B. die Novalung $\subset$ ermöglichen es, den Gasaustausch zu verbessern und gleichzeitig eine lungenprotektive Beatmung durchzuführen. Sie unterstützen also die geschädigte Lunge (Kopp et al. 2004). Schwedische Forscher konnten damit eine Steigerung der Überlebensrate bei ARDS-Patienten erreichen (Linden et al. 2000).

\subsubsection{Pharmakologische Therapie}

Eine direkte, kausale Therapie des ARDS ist bisher nicht sicher möglich. Viele Therapieansätze haben zum Ziel, die stattfindenden inflammatorischen Prozesse abzuschwächen oder zu blockieren. Als weitere supportive Maßnahmen werden eine Senkung des pulmonalen Hochdrucks und eine Surfactantsubstitution angewandt. 


\subsubsection{Antiinflammation}

Bei der Entstehung des ARDS spielen inflammatorische Prozesse eine zentrale Rolle. Hierbei kommt es zur Freisetzung von proinflammatorischen Molekülen, Enzymen, Sauerstoffradikalen und unter anderem zur Einwanderung von Entzündungszellen wie polymorphonukleären Leukozyten (PMNs) (Strieter und Kunkel 1994). Einzelne Studien konnten positive Effekte einer immunsuppressiven Glukokortikoidtherapie bei ARDSPatienten zeigen (Meduri und Chinn 1994, Meduri et al. 1994, Meduri et al. 1998). Eine antioxidative Therapie etwa mit Azetylzystein oder Prozystein ist aufgrund der widersprüchlichen Ergebnisse für unterschiedliche Dosierungen nicht gesichert (Kopp et al. 2003).

\subsubsection{Vasodilatanzien}

Der überwiegende Teil der ARDS-Patienten entwickelt im Verlauf der Erkrankung einen pulmonalen Hypertonus (PH) (Jardin et al. 1979) mit mittleren pulmonalarteriellen Drü cken (MPAP) zwischen 25 und $30 \mathrm{mmHg}$. Ein Anstieg des Gefäßwiderstandes im pulmonalen Stromgebiet tritt ebenfalls bei einer Vielzahl der Patienten auf (Brower et al. 2001). Die Ursachen für eine ARDS-assoziierte pulmonale Hypertonie sind multifaktoriell. Eine Hypoxie- bzw. inflammatorisch bedingte prä- und postkapilläre Vasokonstriktion kommt ebenso in Betracht wie die Kompression pulmonaler Gefäße durch einen hohen PEEP oder durch ein interstitielles Ödem (Brower et al. 2001, Moloney und Evans 2003, Walmrath et al. 1997, Weber-Carstensen und Lohbrunner 1999). Durch die Nachlasterhöhung des rechten Ventrikels wird dessen Auswurfleistung verringert, was zu einer negativen Beeinflussung des Herzzeitvolumens führt (Coetzee et al. 1996, Enger und O'Toole 1991, Foex 1991, Vieillard-Baron et al. 2001). In Verbindung mit dem gestörten Ventilations-Perfusions-Verhältnis $(\mathrm{V} / \mathrm{Q})$ und der folgenden Erhöhung des intrapulmonalen Shunts (Qs/Qt) kommt es möglicherweise zu einer Verringerung der systemischen Sauerstoffversorgung. Hierdurch erhöht sich das Risiko für ein Multiorganversagen. Ist der rechte Ventrikel durch die pulmonale Hypertonie erst einmal dilatiert (cor pulmonale), was bei bis zu 25\% der Patienten mit ARDS vorkommen kann (VieillardBaron et al. 2001), beeinträchtigt dies auch den linken Ventrikel (Jardin et al. 1981). Das Risiko für ein akutes Herz-Kreislauf-Versagen oder eine Verschlechterung der Sauerstoffversorgung steigt. Der erhöhte hydrostatische Druck im Gefäßsystem kann außerdem zur Bildung oder Verstärkung des Lungenödems führen (Lohbrunner et al. 2004). 
Höhe und Dauer der pulmonalen Hypertonie beeinflussen die Letalität der Patienten negativ, und so ist die Senkung des mittleren pulmonalarteriellen Drucks neben der optimalen Oxygenierung ein Baustein der Therapie des ARDS (Zwissler 2002).

\subsubsection{Surfactant}

Das Surfactant der Lunge hat eine zentrale Bedeutung für die Funktion der Alveolen. Da Alveolen dem Gesetz von La Place folgen und dazu neigen zu kollabieren, sind sie auf die Fähigkeit des Surfactant angewiesen, die Oberflächenspannung herabzusetzen (Surfactantprotein-B und Surfactantprotein-C) und die Alveolen damit offen zu halten. Im Rahmen des ARDS kommt es zu einer Schädigung sowie zu einem Verlust von Surfactant bildenden Pneumozyten des Typs II. Zusammen mit dem pulmonalen Ödem ist so die Funktion des Surfactant eingeschränkt bzw. aufgehoben und es kommt leichter zum Kollaps von Alveolen und den damit verbundenen Folgen (Baudouin 2004). Die Substitution von natürlichem und synthetischem Surfactant wird diskutiert und bereits klinisch angewandt (Haitsma et al. 2004, Lewis und Brackenbury 2003). Zwar ließ sich hierdurch eine Verbesserung des Gasaustauschs innerhalb der vierundzwanzigstündigen Behandlung mit Surfactant nachweisen, jedoch führte dies nicht zu einer verbesserten Überlebensrate (Spragg et al. 2004).

\subsubsection{Lagerungstherapie}

Die Beatmung in Bauchlage ist technisch einfach, vergleichsweise ohne großen Aufwand durchführbar und kommt daher oft zur Anwendung (Weber-Carstensen und Lohbrunner 1999). Den Erfolg der Lagerungstherapie erklärt man sich durch eine schwerkraftvermittelte Verschiebung des Lungenödems, eine Umverteilung der Durchblutung, die Erhöhung der Residualkapazität und ein verändertes Ventilationsverhältnis (Weber-Carstensen und Lohbrunner 1999). Bis heute gibt es indes keine klinischen Studien, die eine Verbesserung der Überlebensrate durch Beatmung in Bauchlage belegen, jedoch lässt sich die Oxygenierung verbessern (Piedalue und Albert 2003). Trotzdem gibt es eine Empfehlung zur Anwendung der Bauchlagerung beim ARDS (Kopp et al. 2003). Alternativ besteht die Möglichkeit, den Patienten unter kontinuierlicher Rotation mit bis zu $60^{\circ}$ um die Längsachse zu beatmen. Eine konkrete Empfehlung zur Rotationstherapie kann aufgrund fehlender Studien ebenfalls nicht gegeben werden (Kopp et al. 2003). 


\subsubsection{Schädigungsmodelle}

Um das Acute Respiratory Distress Syndrome besser erforschen zu können, wurden verschiedene Schädigungsmodelle entwickelt, die eine dem ARDS vergleichbare Lungenschädigung im Tierversuch induzieren sollen. Grob lassen sich diese Verfahren in eine endopulmonale Schädigung und eine systemische Schädigung unterteilen. Die endopulmonale Schädigung erfolgt meist über einen saline washout, Inhalation von Gasen oder eine Salzsäureapplikation (Aspirationsmodell). Die systemische Schädigung erfolgt durch die intravenöse Applikation von pulmonalschädigenden Substanzen wie z.B. Ölsäure (Hedlund et al. 1985). Andere Verfahren wie z.B. die operative Anlage von Zwerchfellhernien konnten sich in der Praxis nicht durchsetzen. Ein Nachteil aller Schädigungsmodelle ist das Fehlen standardisierter Vorgehensweisen.

\subsection{Schädel-Hirn-Trauma, Acute Intracranial Hypertension (AICH)}

\subsubsection{Epidemiologie}

Hirnschädigung ist eine der Hauptursachen für körperliche Behinderung und Tod aufgrund eines erlittenen Traumas (Zink 2001) und spielt eine entscheidende Rolle in 50\% aller Todesfälle, die durch Traumata bedingt sind.

Die Inzidenz eines Schädel-Hirn-Traumas (SHT) ist von zahlreichen Faktoren (Alter, Geschlecht, Beruf usw.) abhängig (D'Ambrosio und Perucca 2004). Studien aus den USA geben eine Inzidenz von etwa 1,8 - 2,5 /1000 an; in Europa wird die Inzidenz sogar noch höher eingeschätzt (D'Ambrosio und Perucca 2004).

Das Schädel-Hirn-Trauma ist die Todesursache Nummer eins bei Patienten $<45$ Jahren und die Hauptursache für Tod oder Schwerstbehinderung von Kindern weltweit (Carli und Orliaguet 2004). Die Altersgruppen mit dem höchsten Risiko für ein SchädelHirn-Trauma sind Kinder im Alter von 5 - 9 Jahren und Erwachsene über 80 Jahre (Rao und Lyketsos 2000). Die höchste Sterblichkeit und Hospitalisationszahl aufgrund von Schädel-Hirn-Traumen finden sich bei Personen $>65$ Jahre (Brown et al. 2008). Die höchste Inzidenz jedoch besteht in der Altersgruppe zwischen 15 und 24 Jahren (Hardman und Manoukian 2002). Unabhängig vom Alter sind Männer zweimal häufiger betroffen als Frauen und haben ein vierfach erhöhtes Risiko für ein tödlich verlaufendes Schädel-Hirn-Trauma (Rao und Lyketsos 2000).

Die Sterblichkeit von Patienten mit Schädel-Hirn-Trauma konnte durch verbesserte Behandlungsstrategien in den letzten Jahrzehnten deutlich gesenkt werden. In den siebzi- 
ger Jahren starben ca. 50\% der mit SHT hospitalisierten Patienten, zu Beginn des 21. Jahrhunderts sind es noch ca. 25\% (Zink 2001).

Die Mortalität des SHT wird mit ca. 21\% 30 Tage nach dem Ereignis angegeben (Brown et al. 2008). Ein schweres Schädel-Hirn-Trauma (GCS < 9) hat eine statistische Mortalität von $30-50 \%$ (Park et al. 2008).

\subsubsection{Pathogenese}

Man unterscheidet zwei große Untergruppen von Hirnschädigungen: traumatische (TBI = traumatic brain injury) und nicht-traumatische Schädigungen. Traumatische Hirnschädigungen setzen eine mechanisch-physikalische Komponente als Ursache voraus, wohingegen die nicht-traumatische Schädigung auf endogenen Faktoren wie z.B. Minderperfusion, Entzündung oder Blutung beruhen kann. Zusätzlich unterscheidet man noch zwischen lokal begrenzter oder diffuser Hirnschädigung.

Anders als die meisten traumatisch bedingten Todesfälle sterben Patienten mit traumatischem Hirnschaden nicht sofort, sondern überwiegend erst Tage oder Wochen nach dem erlittenen Trauma (Sauaia et al. 1995). Anstatt dass es im Rahmen des stationären Aufenthaltes zu einer zügigen Besserung der Symptomatik kommt, verschlechtert sich der Zustand bei ca. 40\% der Patienten zunächst (Narayan et al. 2002). Schäden, die direkt mit dem Ereignis in zeitlichem Zusammenhang stehen (z.B. Blutung, Quetschung), liefern keine suffiziente Erklärung für diese progrediente Verschlechterung. Vielmehr ist hierfür eine biochemische Kaskade verantwortlich, die dem Akutereignis zeitlich versetzt folgt (Verweij et al. 2000). Diese sekundären Prozesse können den primär entstandenen Schaden nachträglich dramatisch verschlechtern (Park et al. 2008) und sind hauptverantwortlich für die stationären Todesfälle durch traumatische Hirnschädigung (Ghajar 2000).

Teil dieser Entzündungskaskade sind unter anderem Entzündungsmediatoren wie Interleukin-6, Tumor-Nekrose-Faktor alpha, Schäden an der Blut-Hirn-Schranke, freie Radikale, überschießende Freisetzung von Neurotransmittern, Calcium- und Natriumeinstrom in Neurone sowie mitochondriale Dysfunktion (Park et al. 2008).

Weitere Faktoren für eine Verschlechterung sind Ischämie, cerebrale Hypoxie, erniedrigter cerebraler Perfusionsdruck, cerebrales Ödem und erhöhter intrakranieller Druck. Der intrakranielle Druck kann z.B. durch eine Raumforderung (akute Blutung) steigen und zu einer Minderperfusion aufgrund eines nicht mehr ausreichenden cerebralen Perfusionsdrucks führen. Die Folge ist eine nicht suffiziente Sauerstoffversorgung des Gehirns (Ghajar 2000). 
Tabelle 2: Einteilung des Schweregrades eines Schädel-Hirn-Traumas

\begin{tabular}{llll} 
Schweregrad & GCS & PTA & LOC \\
\hline mild & $13-15$ & $<1$ Stunde & $<30 \mathrm{~min}$ \\
moderat & $9-12$ & $60 \mathrm{~min}-24 \mathrm{~h}$ & $60 \mathrm{~min}-24 \mathrm{~h}$ \\
schwer & $3-8$ & $>24 \mathrm{~h}$ & $>24 \mathrm{~h}$
\end{tabular}

GCS = Glasgow Coma Scale; PTA = post traumatic amnesia; $L O C=$ loss of conscious ness

Hirnschäden können nach Ihrer Schwere in drei Gruppen unterteilt werden: mild, moderat oder schwer. Im akuten Stadium der Schädigung hat sich die Glasgow Coma Scale (GCS) zur ersten Einschätzung des Ausmaßes eines Schädel-HirnTraumas etabliert.

\subsubsection{Therapie}

Zurzeit gibt es keine gesicherte Möglichkeit der kausalen Therapie der Sekundärfolgen eines Schädel-Hirn-Traumas (Park et al. 2008). Die verschiedenen Pathomechanismen liefern jedoch Ansatzpunkte für Therapieoptionen (cerebraler Perfusionsdruck, intrakranieller Druck, Oxygenierung usw.), die eine Progredienz verlangsamen oder verhindern können (Zink 2001).

Im Gegensatz zu vielen anderen Verletzungen ist es bei traumatischen Hirnschäden schwer, die Verletzung von Gewebe durch operative Maßnahmen oder medikamentöse Therapien rückgängig zu machen bzw. eine restitutio ad integrum zu erreichen. Vorrangiges Ziel der initialen Behandlung ist es daher, den Patienten zunächst zu stabilisieren und eine weitere Schädigung bzw. ein Fortschreiten zu verhindern, um überlebensfähige Zellen im Bereich der Penumbra zu erhalten (NIH 2008). Hauptansatzpunkte der Therapie sind die Versorgung des Gehirns mit Sauerstoff, die Aufrechterhaltung einer adäquaten Perfusion, die Überwachung des Blutdrucks, die endotracheale Intubation und damit die Sicherung der Atemwege und Ventilation des Patienten sowie die Überwachung und Therapie des intrakraniellen Drucks (Zink 2001). Die Überwachung des intrakraniellen Drucks hat hierbei einen hohen Stellenwert (Park et al. 2008). Die Therapie des erhöhten ICP umfasst sowohl technisch einfache Maßnahmen wie z.B. die günstige Lagerung des Patienten in Oberkörper-30-hoch-Position (Schneider et al. 1993) als auch aufwendige und invasive Methoden wie z.B. die Ventrikulostomie, die Hemikraniektomie u.a.m. (Valadka 2004). Weniger invasive Maßnahmen umfassen die Anwendung von Sedativa, Relaxanzien, Osmotherapie und milde Hyperventilation 
(Salomone und Frame 2004). Die Hyperventilation führt aufgrund einer durch niedrige $\mathrm{CO}_{2}$-Konzentrationen bedingten Vasokonstriktion zu einem verminderten Blutfluss zum Gehirn und somit zu einer Verminderung des intrakraniellen Drucks, birgt aber auch die Gefahr einer Ischämie und verschlechtert, präventiv über einen längeren Zeitraum angewandt, das Outcome des Patienten (Ghajar 2000).

Besonders wichtig ist daher auch die präklinische Entscheidung, den Patienten in ein spezialisiertes Zentrum zu transportieren (Salomone und Frame 2004).

\subsection{Schädel-Hirn-Trauma und Acute Respiratory Distress Syndrome}

Die Inzidenz von pulmonalen Funktionsstörungen bei Patienten mit Schädel-HirnTrauma wird aktuell mit 5-30 \% angegeben (Bratton und Davis 1997, Contant et al. 2001, Holland et al. 2003, Kahn et al. 2006, Mascia et al. 2007, Piek et al. 1992, Wartenberg et al. 2006, Zygun et al. 2005). Schaut man sich die zitierten Studien genauer an, so wird klar, dass diese Schwankungsbreite mit einem sehr unterschiedlichen Patientengut und teilweise unterschiedlichen Kriterien für eine akute Lungenschädigung (ALI) oder ein ARDS verknüpft ist. So sind in vielen Studien Patienten mit verschiedenen Schweregraden eines Schädel-Hirn-Traumas eingeschlossen worden. Begrenzt man sich auf Patienten mit einem schweren SHT (GCS < 9), so liegt die Inzidenz von akuten Lungenschäden (ALI) bei 20-30\% und die von akutem Lungeversagen (ARDS) bei $5-10 \%$.

In einer Studie von Zygun et al. (Zygun et al. 2005) wurde die Häufigkeit nicht neurologischer Organstörungen bei schwerem Schädel-Hirn-Trauma untersucht. Dabei entwickelte sich bei $81 \%$ der Patienten eine respiratorische Funktionsstörung und bei $23 \%$ eine respiratorische Insuffizienz oder gar ein respiratorisches Versagen.

In einer Studie von Bratton und Davis (Bratton und Davis 1997) konnte gezeigt werden, dass bei $20-25 \%$ der Patienten mit isoliertem Schädel-Hirn-Trauma eine respiratorische Insuffizienz mit einem $\mathrm{paO}_{2} / \mathrm{FiO}_{2}$ Verhältnis von $<300 \mathrm{mmHg}$ vorlag (ALI-Kriterien erfüllt).

Zur Entstehung einer pulmonalen Funktionsstörung bei einem vorliegenden traumatischen Hirnschaden gibt es verschiedene Theorien. Man subsumiert sie unter dem Begriff „Neurogenes Pulmonales Ödem“. Zum einen spielt hierbei möglicherweise eine Aktivierung des sympathischen Nervensystems nach akutem Schädel-Hirn-Trauma eine Rolle (Theodore und Robin 1976), zum anderen wird eine Entzündungskaskade als weiterer Auslöser eines neurogenen pulmonalen Ödems diskutiert (Lucas et al. 2006). 
Der hohe intrakranielle Druck, der durch die traumatische Hirnschädigung entsteht, erfordert zur ausreichenden Perfusion des Gehirns einen hohen systemischen arteriellen Mitteldruck (MAP), um einen ausreichenden cerebralen Perfusionsdruck aufrechtzuerhalten. Dieser, so wird vermutet, kann durch Erhöhung des hydrostatischen Drucks im Gefäßlumen der pulmonalen Gefäßstrombahn im Verlauf der Therapie ebenfalls zu einem ARDS führen (Contant et al. 2001).

In einer Studie von Holland et al. zur Inzidenz von ARDS bei Patienten mit SchädelHirn-Trauma und zum Einfluss des ARDS auf die Sterblichkeit und das neurologische Outcome konnte gezeigt werden, dass bei Patienten mit traumatischer Hirnschädigung, die ein ARDS entwickeln, die Mortalität und das schlechtere neurologische Outcome signifikant höher waren als bei Patienten ohne ein ARDS (Holland et al. 2003). Ein Zusammenhang zwischen dem Schweregrad des Schädel-Hirn-Traumas und der Entwicklung eines ARDS konnte in dieser Studie jedoch nicht nachgewiesen werden.

\subsection{Aufgabenstellung}

Ziel der vorliegenden Arbeit ist die Untersuchung möglicher Auswirkungen eines akut erhöhten intrakraniellen Drucks auf gesunde und geschädigte Schweinelungen durch computertomographisch gestützte Dichtemessungen, Bestimmung des extravaskulären Lungenwassers sowie hämodynamischer Messparameter (HF, SV, HZV), arterieller Blutgasanalysen und histologischer Untersuchungen des Lungengewebes.

Die Letalität des isolierten Schädel-Hirn-Traumas (SHT) liegt heute je nach Schweregrad bei ca. 15\%, während sie in Verbindung mit einem Acute Respiratory Distress Syndrome (ARDS) signifikant höher ist (Bratton und Davis 1997, Mascia et al. 2008, Zygun et al. 2005). Neben der hohen Letalität bei Patienten mit Schädel-Hirn-Trauma und Acute Respiratory Distress Syndrome ist auch das neurologische Outcome deutlich schlechter (Mascia et al. 2008), als man es durch die Hirn- und Lungenschädigung allein erklären kann. Im Verlauf zeigen diese Patienten erhebliche psychosoziale Probleme und eine Reduktion ihrer kognitiven Fähigkeiten (Holland et al. 2003).

Es gibt verschiedene Theorien darüber, wie es zu einer ausgeprägten pulmonalen Dysfunktion durch akute intrakranielle Druckerhöhung kommen könnte. Einerseits werden hydrostatische Kräfte, ausgelöst durch eine Erhöhung der Sympathikusaktivität mit nachfolgender Vasokonstriktion und Veränderung der kapillären Permeabilität (Touho et al. 1989, Urdaneta und Layon 2003), dafür verantwortlich gemacht, andererseits geht man von einer akuten, systemischen, inflammatorischen Reaktion aus, die zur medi- 
atorvermittelten Einwanderung von Entzündungszellen in die Lunge und anschließender endothelialer Dysfunktion führen könnte (Kalsotra et al. 2007, Mascia 2009). Beide Theorien lassen sich unter dem Begriff neurogenes pulmonales Ödem (NPÖ) zusammenfassen (Mascia 2009).

Die pathogenetischen Zusammenhänge beider Krankheitsbilder sind bisher nicht im Detail bekannt. Mit der geplanten Untersuchung sollen an einem Tiermodell mögliche pathophysiologische Zusammenhänge erfasst und mögliche Faktoren dieser Interdependenz erkannt werden.

Mit der Methode der computertomographischen Bestimmung der Lungendichte sowie des extravaskulären Lungenwasserindexes (EVLWI) mittels Thermodilution, histopathologischen Untersuchungen und der beobachtbaren Veränderungen in kranken und gesunden Schweinelungen mit und ohne intrakranielle Druckerhöhung sollen unter Berücksichtigung der Hämodynamik und des Gasaustauschs mögliche Zusammenhänge zwischen diesen Entitäten erkannt und beschrieben werden. 


\section{Material und Methoden}

\subsection{Tiere}

\subsubsection{Versuchstiere}

Alle Versuchsvorhaben wurden durch das Tierversuchs-Komitee der Universität Göttingen genehmigt. Für die Versuche wurden 28 ausschließlich weibliche Schweine (Läufer), Typ „Göttinger Hausschwein“ mit einem mittleren Gewicht von 61 kg (52-65 kg) verwendet. Bereitgestellt wurden diese Versuchstiere vom landwirtschaftlichen Versuchsgut der Universität Göttingen in Relliehausen.

Die Tiere wurden in der zentralen Tierversuchseinrichtung des Universitätsklinikums Göttingen gehalten und von fachkundigem Personal gepflegt. Von dort wurden sie für die Durchführung der Versuche in den benachbarten Tier-OP verbracht.

Die Verpflegung der Schweine erfolgte mit einer hauseigenen Getreidemischung, die zweimal täglich angeboten wurde. Trinkwasser wurde den Tieren ad libitum zur Verfügung gestellt. Die Temperatur der Stallungen wurde konstant bei $23^{\circ} \mathrm{C}$ gehalten.

\subsubsection{Tierversuchsantrag}

Der Tierversuchsantrag wurde bei der niedersächsischen Tierschutzkommission am Landesamt für Verbraucherschutz und Lebensmittelsicherheit in Braunschweig eingereicht und bewilligt.

\subsection{Versuchsaufbau}

\subsubsection{Messturm}

Der Messturm (siehe Abbildungen 3 und 4) wurde eigens für dieses Versuchsvorhaben entworfen. Er wurde mobil konzipiert, trägt alle Überwachungsgeräte und versorgt diese mit der nötigen Spannung, wobei auch während des Transports eine unterbrechungsfreie Spannungsversorgung und damit eine kontinuierliche Datenaufzeichnung gewährleistet sind. Ein integrierter Laptop sicherte die Datenaufzeichnung in Echtzeit. Alle physiologischen Signale wurden über einen geeigneten Analog/Digital-Wandler in Echtzeit digitalisiert, synchron auf einer Zeitachse visualisiert und in einem allgemein zugänglichen Dateiformat gespeichert. 
Abbildung 3: Schematische Darstellung des Messturms

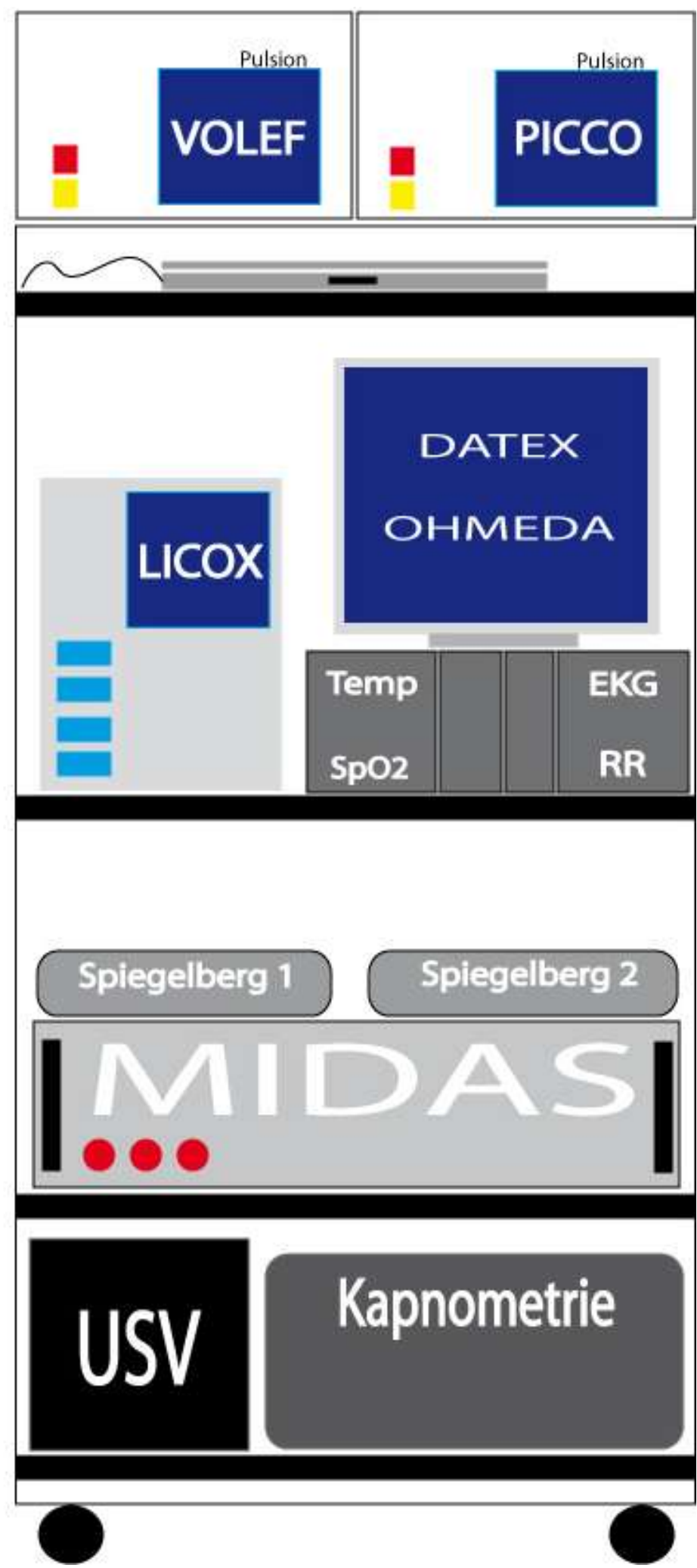

Die Abbildung zeigt den schematischen Aufbau der transportablen Messeinheit inklusive aller Messgeräte, Analog-Digital-Wandler, unterbrechungsfreier Stromversorgung und Laptop. 
Abbildung 4: Messturm

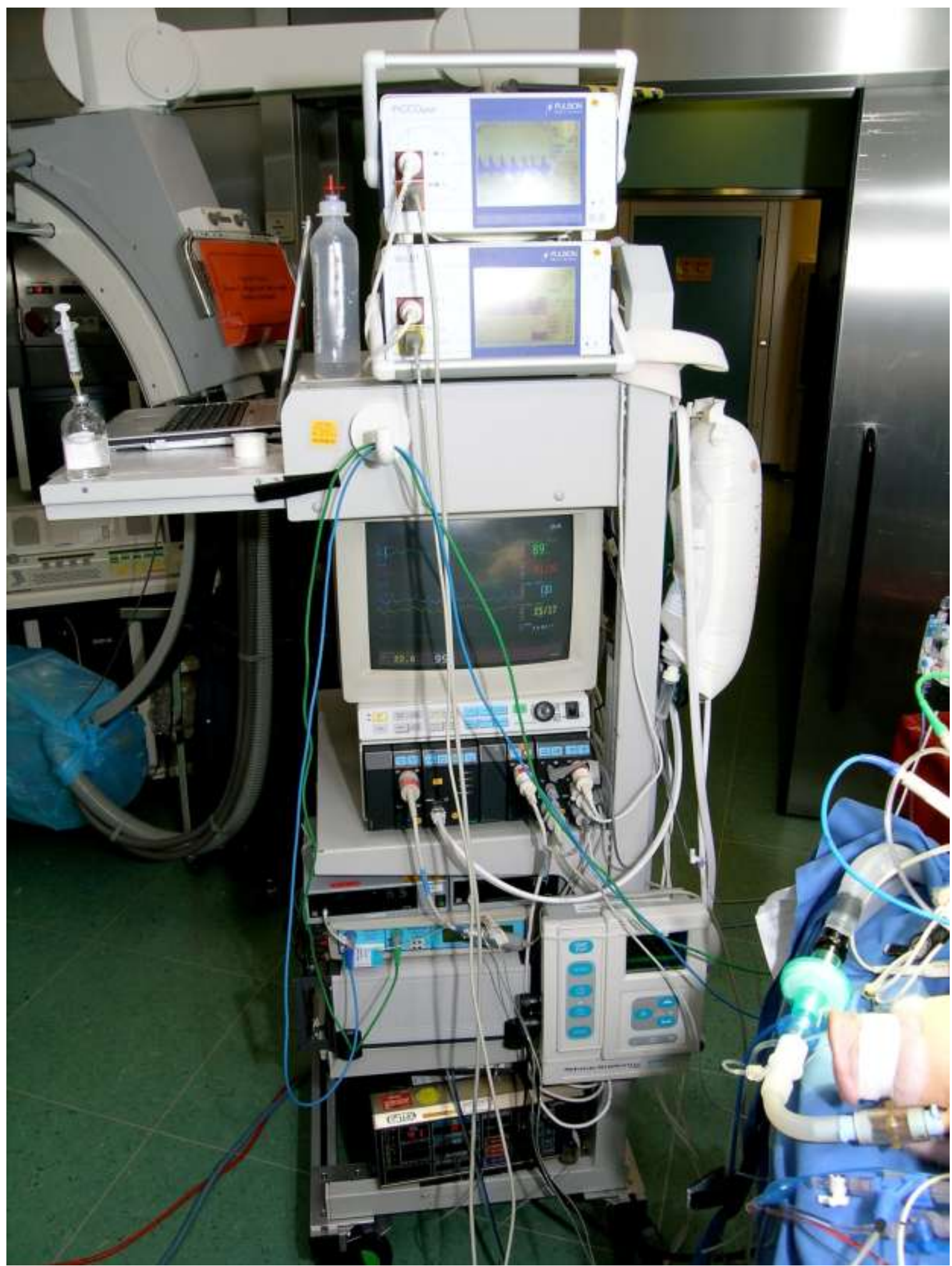

Abbildung des Messturms wie in der Schemazeichnung aus Abbildung 3 dargestellt. 


\subsubsection{Transporteinheit}

Die Transporteinheit (siehe Abbildungen 6 und 7) beherbergt das Schwein während des Transports vom Tier-OP zum Computertomographen. Sie besteht aus einem fahrbaren Stahlrahmen und einer eigens zu diesem Zweck angefertigten röntgendurchlässigen Kunststoffwanne, in der das Schwein positioniert wird. Mit Hilfe der Wanne kann das Schwein zu den Messpunkten auf den CT-Tisch gelagert werden, ohne dass dafür die Messung der hämodynamischen sowie der Gasaustauschparameter unterbrochen werden müsste. Besonders wichtig war hierbei eine stabile Lage des Versuchstiers über die gesamten 240 Minuten. Ein Kippen oder Drehen des Thorax hätte möglicherweise einen Einfluss auf die Verteilung des pulmonalen Ödems oder die Hämodynamik haben können. Die halbkreisförmige Wanne verhinderte durch seitliche Stabilisierung eine Rotation um die Längsachse in Bauchlage (siehe Abbildung 5).

Ein ebenfalls an der Transporteinheit befestigtes Beatmungsgerät mit zwei Sauerstoffflaschen sichert die Sauerstoffversorgung des Versuchstiers auch während des Transports.

Für den Fall eines Stromausfalls verfügt die Transporteinheit über eine integrierte unterbrechungsfreie Stromversorgung.

Abbildung 5: Positionierung des Schweins

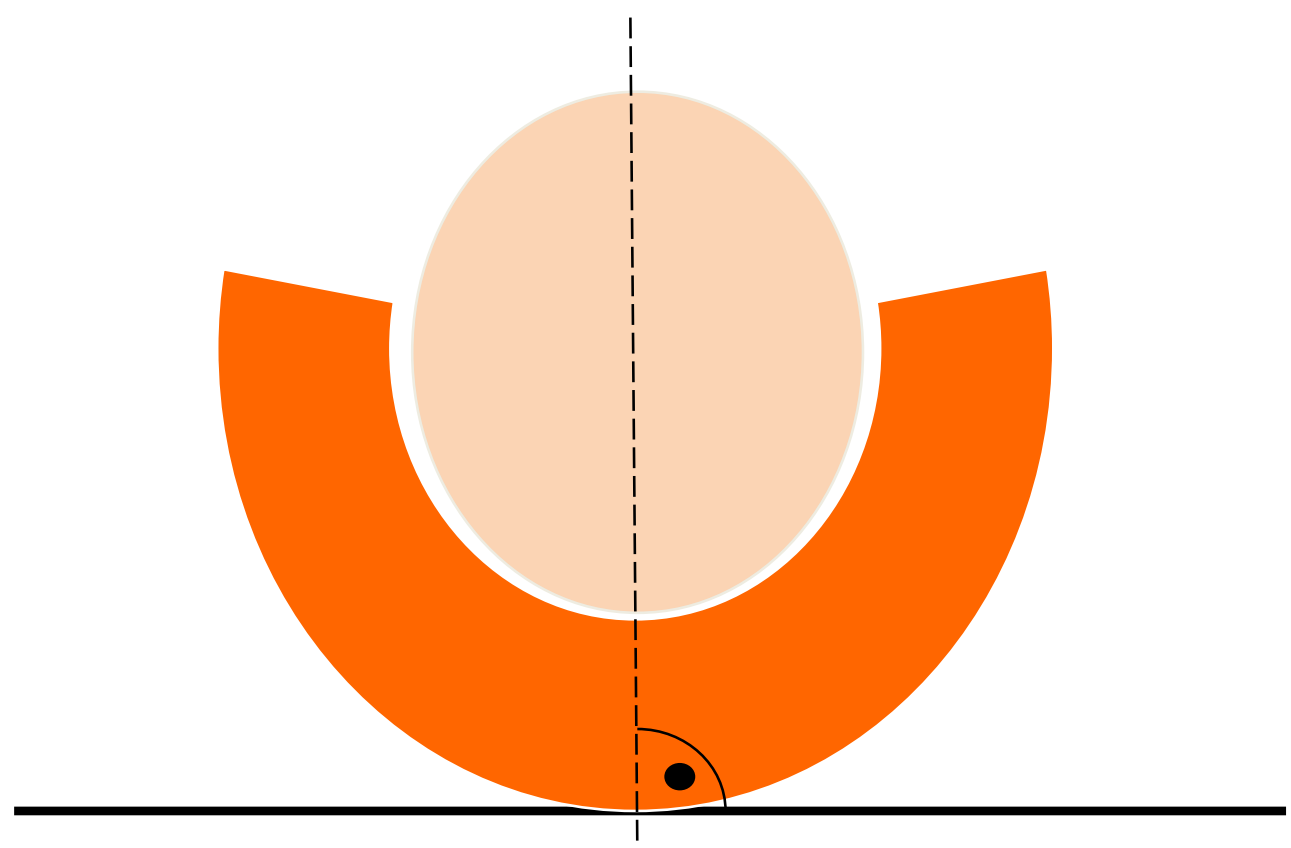

Positionierung des Schweinethorax im rechten Winkel zur Horizontalen. So konnte einer möglicherweise ungleichen Verteilung des Lungenödems entgegengewirkt werden. 
Abbildung 6: Schematische Darstellung der Transporteinheit

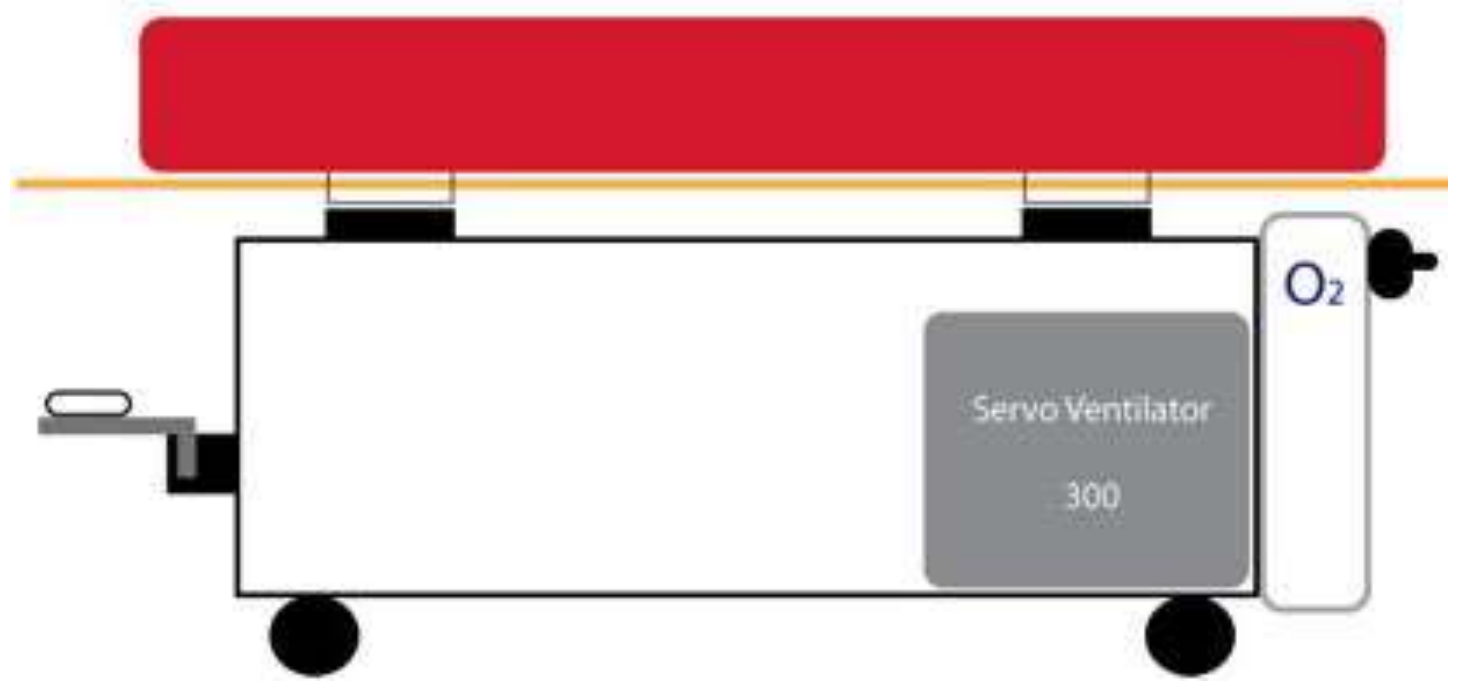

Die Abbildung zeigt den schematischen Aufbau der Transporteinheit inklusive röntgendurchlässiger Wanne, Beatmungsgerät und Sauerstoffversorgung.

Abbildung 7: Versuchstier mit Messturm und Transporteinheit

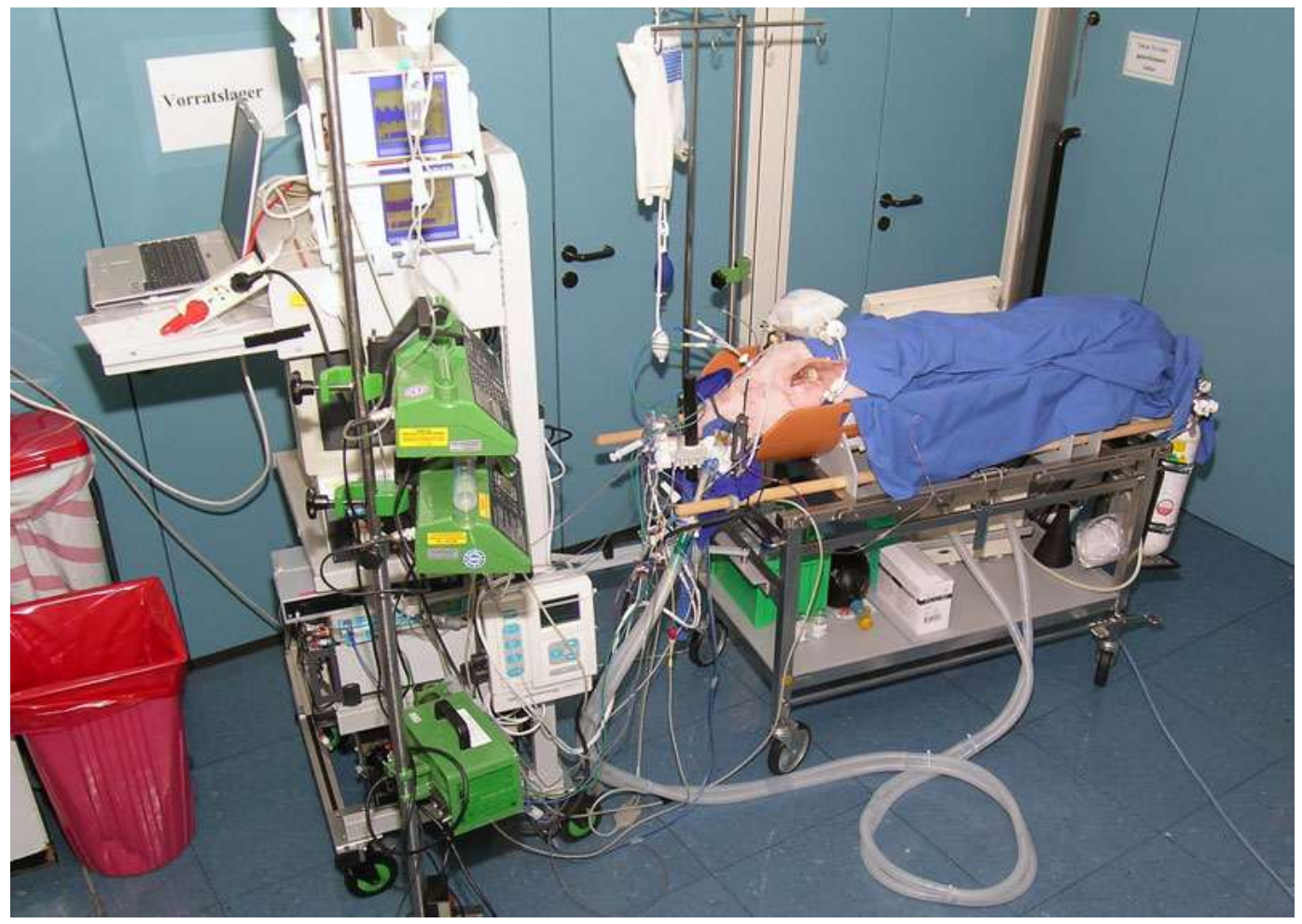

Abbildung von Messturm und Transporteinheit in Aktion. 


\subsection{Versuchsvorbereitungen}

\subsubsection{Allgemeine Versuchsvorbereitungen}

Zu Beginn jedes Versuches wurden zunächst sämtliche elektronischen Geräte mit Hilfe einer Checkliste auf ihre Funktionstüchtigkeit und Vollständigkeit überprüft. Es wurden sterile Druckschläuche angebracht und neue Sauerstoffflaschen an die Transporteinheit montiert.

\subsubsection{Herstellung von Injektionslösungen}

\subsubsection{Narkotika/Analgetika/Hypnotika}

Um eine lang andauernde Narkose aufrechterhalten zu können, wurden vor Beginn des Versuches Perfusoren mit folgenden Medikamenten vorbereitet:

- Ketamin

- Midazolam.

Nach luftblasenfreiem Anschluss der Dreiwegehähne und Perfusorleitungen wurden die Spritzen in den Perfusoren platziert.

\subsubsection{2 Ölsäure}

Ölsäure wurde zur Induktion des ARDS benötigt und in einer Konzentration von 0,1 $\mathrm{ml} / \mathrm{kg} \mathrm{KG}$ mit einer handelsüblichen 20-ml-Spritze (Braun, Melsungen) über den zentralen Venenkatheter verabreicht.

\subsubsection{Vorbereitung des Versuchstiers}

Die weiblichen Hausschweine wurden ca. drei Tage vor dem geplanten Eingriff angeliefert. 24 Stunden vor dem Versuch wurde, zwecks Vermeidung einer Aspiration unter Narkose, Nahrungskarenz eingehalten, nicht jedoch Wasserkarenz. Alle Tiere wurden vor Beginn des Versuchs gewogen und auf mögliche Verletzungen untersucht. Anschließend wurden die Tiere zur Narkoseeinleitung mittels einer Transportbox und unter Mithilfe des Fachpersonals in den Tier-OP 2 der Universitätsklinik Göttingen gebracht.

\subsubsection{Narkoseeinleitung und Aufrechterhaltung}

Nach Prämedikation mit 40 mg i.m. Azaperon (Stresnil, Janssen, Österreich) wurde eine Ohrvene mittels Venenverweilkanüle punktiert und die Narkose durch Bolusinjektion von Thiopental (3-5 mg/kg KG i.v.) sowie $4 \mathrm{mg} / \mathrm{kg} \mathrm{KG}$ Ketamin i.v. (Ketaminol, Vetpharma, Zürich, Schweiz) eingeleitet. Anschließend wurden alle Tiere endotracheal 
intubiert (Portex 6.5, Portex, Deutschland) und maschinell beatmet (Servo 300, Siemens, Deutschland). Die Beatmung erfolgte volumenkontrolliert mit einem Tidalvolumen $\left(\mathrm{V}_{\mathrm{t}}\right)$ von $8 \mathrm{ml} / \mathrm{kg} \mathrm{KG}$ (lungenprotektiv), einem PEEP von $5 \mathrm{~cm} \mathrm{H} \mathrm{H}_{2} \mathrm{O}$, einem Inspirations-/ Exspirationsverhältnis (I:E) von 1:2 und einer $\mathrm{FiO}_{2}$ von 1,0 . Das $\mathrm{V}_{t}\left(\mathrm{~V}_{\mathrm{t}} \max =10 \mathrm{ml} / \mathrm{kg}\right.$ $\mathrm{KG}$ ) sowie die Atemfrequenz wurden zwecks Aufrechterhaltung einer arteriellen $\mathrm{CO}_{2}$ Konzentration $\left(\mathrm{PaCO}_{2}\right)$ unter $60 \mathrm{mmHg}$ entsprechend angepasst. Überwacht wurde das end-tidale $\mathrm{CO}_{2}$ mit Hilfe eines Kapnographen (Datex Capnomac Ultima, Finland). Die Narkose wurde durch kontinuierliche Infusion von Ketamin $(10 \mathrm{mg} / \mathrm{kg} / \mathrm{h})$ und Midazolam $(1 \mathrm{mg} / \mathrm{kg} / \mathrm{h})$ über das gesamte Experiment aufrechterhalten, um einen gleichmäßig schmerzfreien Zustand der Tiere zu gewährleisten.

Um einen ausgeglichenen Flüssigkeitshaushalt zu erreichen, wurde allen Tieren RingerAcetat-Lösung mit einer Infusionsrate von 3-4 ml/kg/h verabreicht. Zusätzlich erhielten alle Tiere ein Basismonitoring in Form von EKG, nicht invasiver Blutdruckmessung und Messung der peripheren Sauerstoffsättigung (Datex Ohmeda, Finland).

\subsubsection{Lagerung des Tieres}

Die Einleitung der Narkose erfolgte in Bauchlage. Nach abgeschlossener Narkoseeinleitung wurde das Versuchstier in Rückenlage gedreht und es wurde mit der Anlage der Messsonden und Katheter begonnen (siehe Abbildung 8). Nach Platzierung aller Katheter und Messsonden wurde das Tier für den Rest des Versuchsablaufs in Bauchlage gebracht (siehe Abbildung 9). 
Abbildung 8: Lagerung im Tier-OP 2

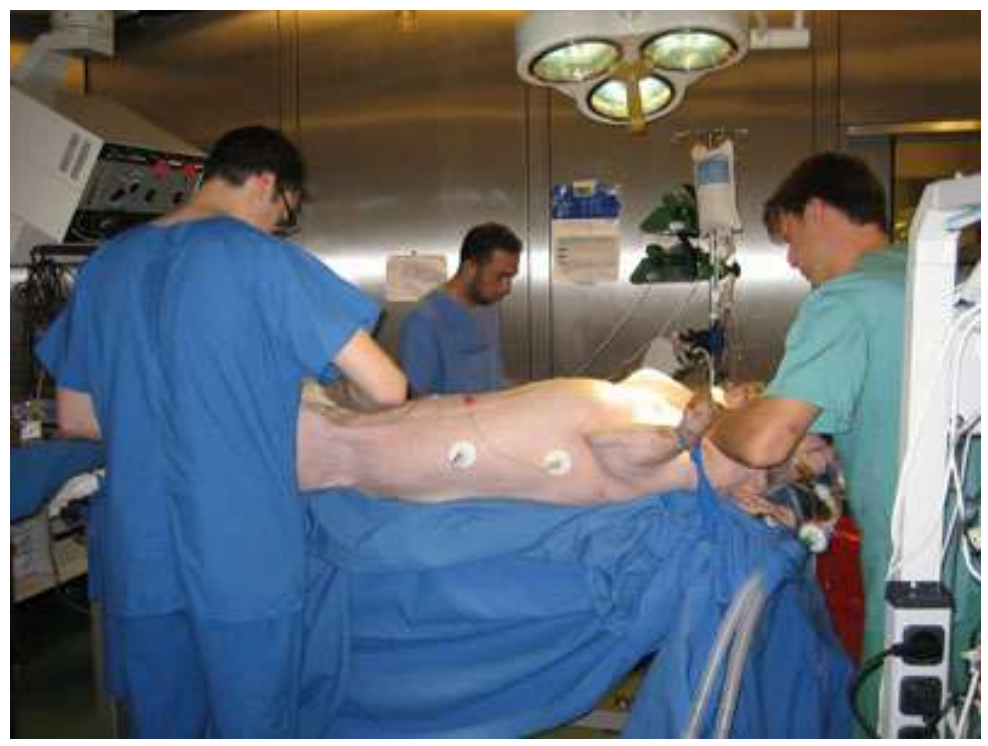

Abbildung 9: Lagerung im GE Lightspeed VCT

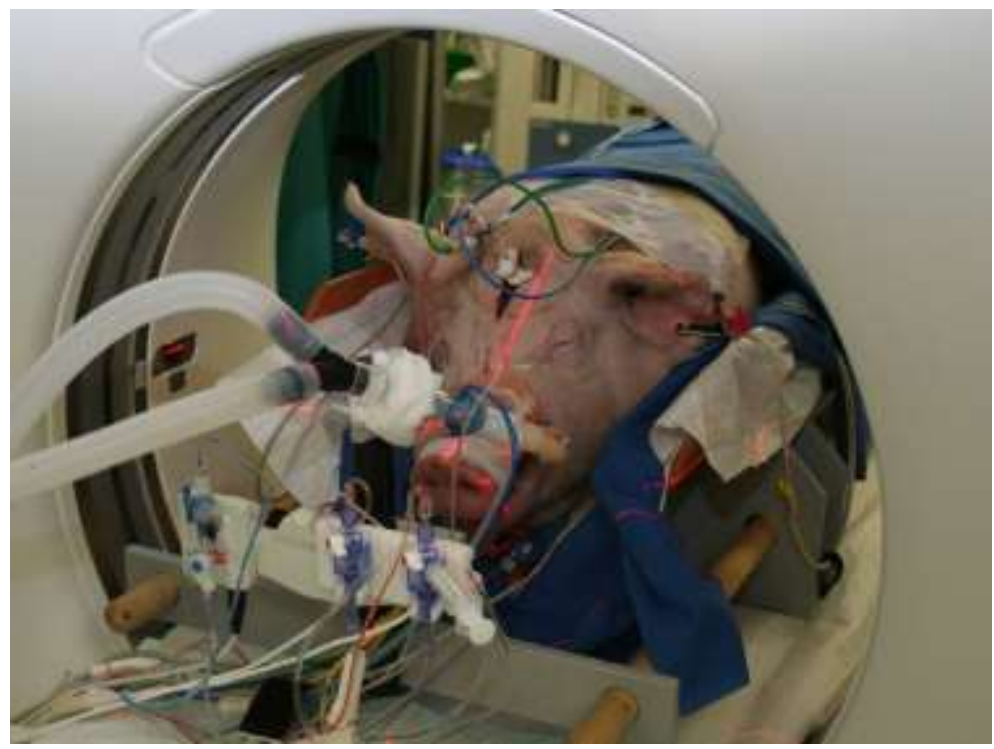

\subsubsection{Messung von Hämodynamik und Gasaustausch}

Nach Rückenlagerung und steriler Abdeckung erfolgte über die Punktion der Arteria femoralis die Anlage eines fiberoptischen Thermistor-Katheters (Pulsiocath, 4F FT PV 2024; Pulsion Medical Systems, München) mittels Seldinger-Technik (siehe Abbildung 11). Durch Punktion im lateralen Halsdreieck wurde ein 5-French-Katheter in die Vena jugularis interna eingeführt, über den im Anschluss ein Swan-Ganz-Katheter (Volef, Pulsion Medical Systems, München) platziert wurde. Die genaue Lage des Katheters wurde druckkontrolliert bestimmt. Die Katheter wurden mittels Druckaufnehmern an einen Monitor (PiCCO, Volef, Pulsion Medical Systems, München) angeschlossen und 
vor Versuchsbeginn zur Atmosphäre hin genullt. Die Aufzeichnung der Messdaten erfolgte in Echtzeit (siehe Abbildung 10) über einen Laptop mit Hilfe des Programms PiCCOwin (Pulsion Medical Systems, München) in eine Microsoft Access Datenbank (Microsoft Corporation, Redmond, USA). Protokolliert wurden Herzzeitvolumen (HZV), Schlagvolumen (SV), mittlerer arterieller Druck (MAP) und mittlerer pulmonalarterieller Druck (MPAP), extravaskulärer Lungenwasserindex (EVLWI) und das intrathorakale Blutvolumen (ITBI). Die Messungen und Kalibrierungen erfolgten zu fest definierten Zeitpunkten mittels Thermodilution durch dreifache zentralvenöse Injektion von $20 \mathrm{ml}$ kalter $\left(<8^{\circ} \mathrm{C}\right) 0,9 \%$ Kochsalzlösung.

Ebenfalls in regelmäßigen Zeitabständen wurden durch Entnahme arterieller und gemischt-venöser Blutproben die Blutgase bestimmt. Zum Einsatz kam hier ein Radiometer vom Typ ABL 510 (Radiometer, Copenhagen, Dänemark).

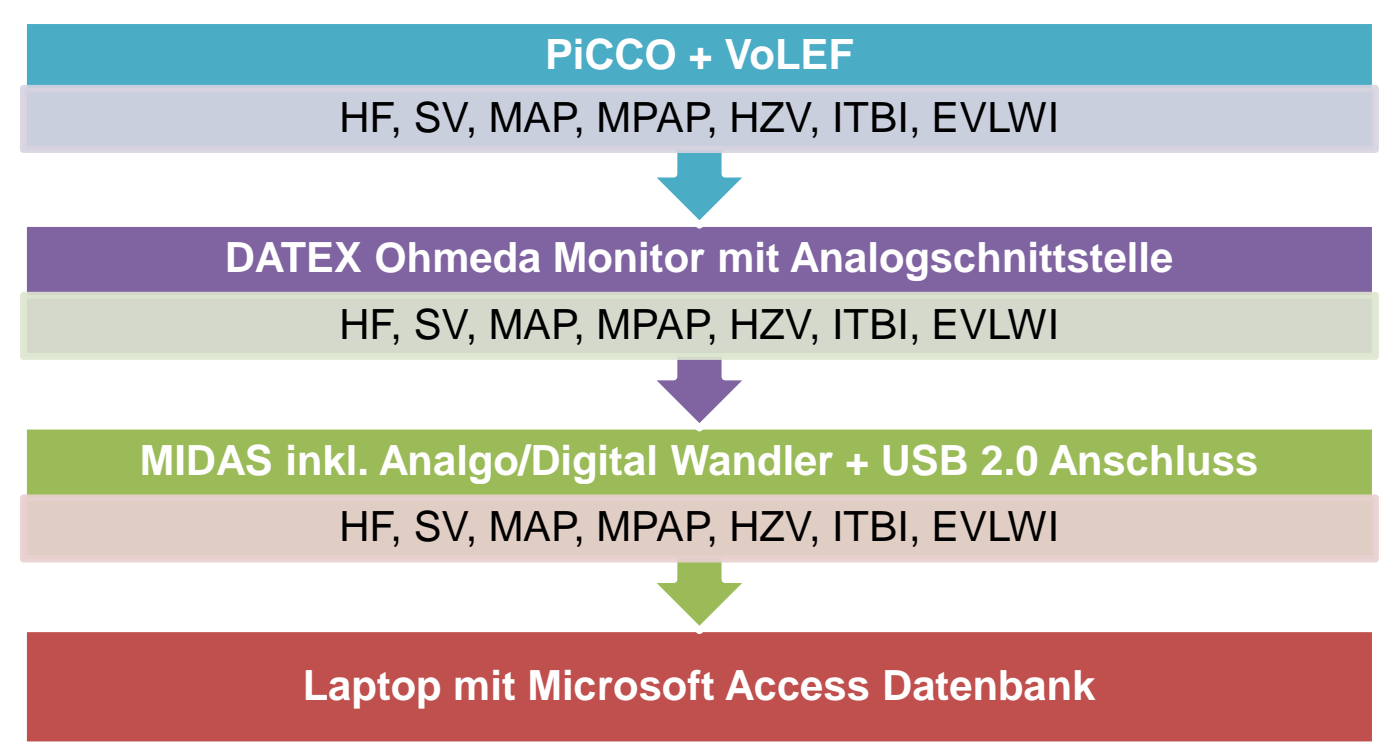

Abbildung

10: Datenverarbeitung der Hämodynamik

Fluss der hämodynamischen Messdaten von der Erfassung bis zur endgültigen Aufzeichnung. 
Abbildung 11: Ultraschallgesteuerte Anlage eines PICCO-Katheters
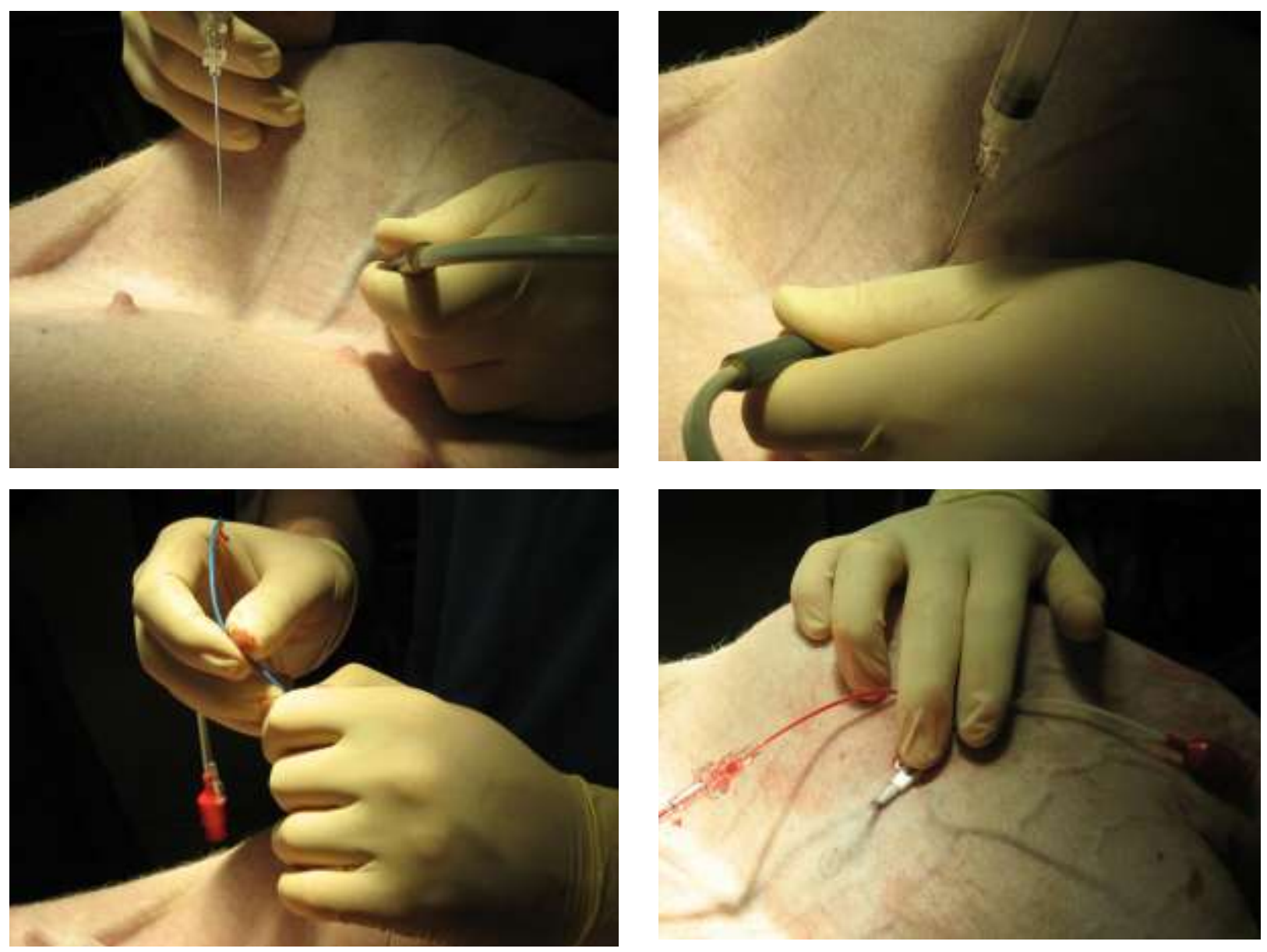

Ultraschallgesteuerte Punktion der linken Arteria femoralis und Anlage eines PiCCO-Katheters mittels Seldinger-Technik. 


\subsubsection{Intracerebrale Druckmessung}

Im Anschluss an die venöse sowie die arterielle Katheterisierung erfolgte die Anlage einer intracerebralen Messsonde, über die der cerebrale Sauerstoffgehalt $\left(\mathrm{PtiO}_{2}\right)$, der cerebrale pH-Wert sowie der intracerebrale Druck (ICP) gemessen wurden. Hierfür wurde ein Bohrloch rechts frontal angelegt. Nach Perforation der Dura mit Hilfe einer Sonde konnte eine Fixierschraube im Knochen verankert werden. Durch diese wurden dann eine Sauerstoffsonde, eine ICP-Sonde und eine Temperatursonde platziert (Integra Neuroscience, Integra $\mathrm{GmbH}, 40880$ Ratingen, Deutschland). Die Sonden kamen ungefähr $25 \mathrm{~mm}$ unterhalb der Dura in der weißen Substanz zum Liegen (siehe Abbildung 13). Die ICP Sonde wurde mit einem Camino ICP Monitor (Integra Neuroscience, Integra $\mathrm{GmbH}, 40880$ Ratingen, Deutschland), Temperatur- sowie Sauerstoffsonde an einen „Licox Brain Tissue Oxygen Monitor" angeschlossen. Die Sonden wurden in ihrer Position fixiert und das Bohrloch mittels Knochenzement abgedichtet. Die Kalibrierung der Geräte erfolgte nach den genauen Vorgaben des Herstellers. Im Rahmen dieser Arbeit war lediglich die intracerebrale Druckmessung relevant. Die Daten wurden mit Hilfe eines Laptops (Samsung Electronics) über die gesamte Versuchsdauer aufgezeichnet (siehe Abbildung 12).

Abbildung 12: Datenverarbeitung intracerebrale Druckmessung

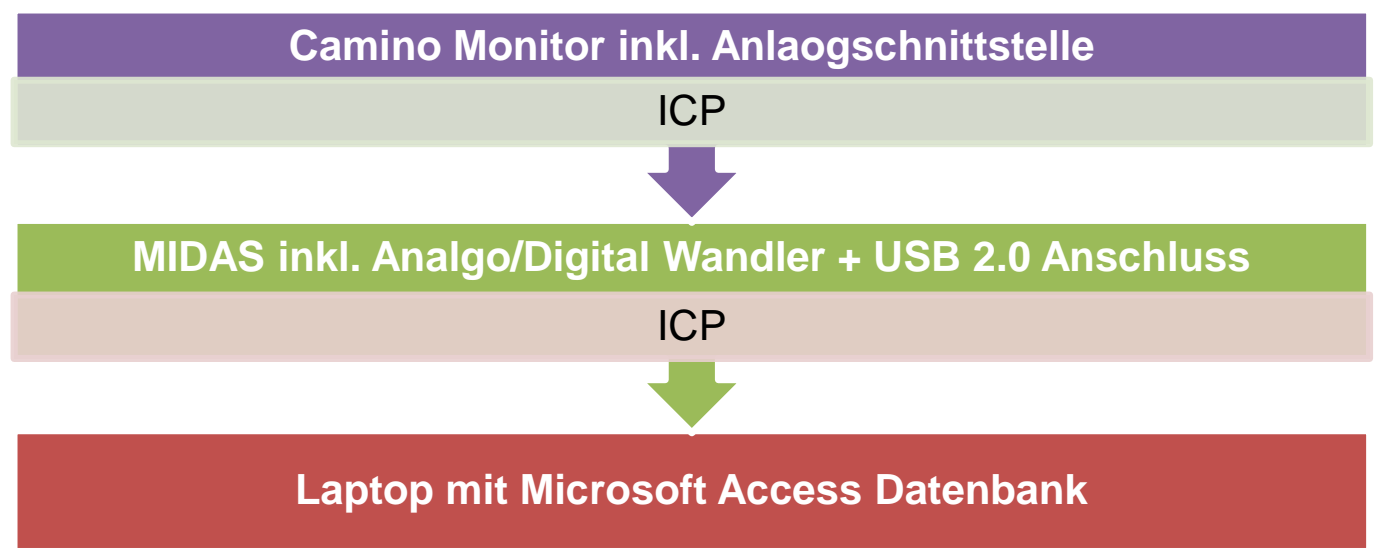

Fluss der intrakranialen Druckmessung von der Erfassung bis zur endgültigen Aufzeichnung. 
Abbildung 13: Platzierung der intrakraniellen Sonden mittels Bohrlochtrepanation
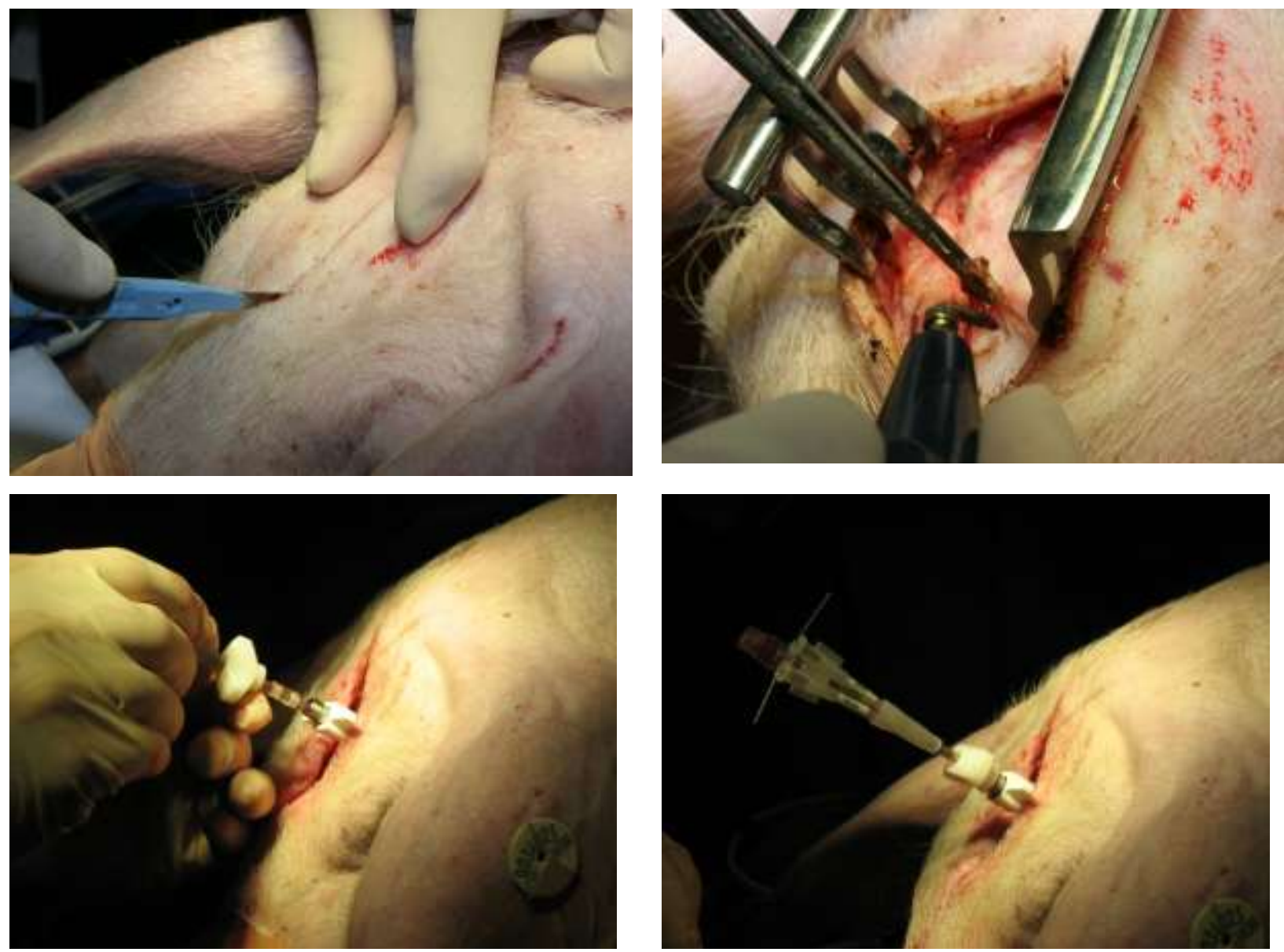

Anlage der intracerebralen Druckmessung. Zunächst wurde ein Längsschnitt auf Höhe der vermuteten Sutura sagittalis vorgenommen. Danach wurde die Schädeldecke freipräpariert, um die Sutura sagittalis sichtbar zu machen. Sie dient als anatomische Landmarke, um eine Punktion des Sinus sagittalis zu vermeiden. Es folgte die Bohrlochtrepanation und Platzierung der intracerebralen Druckmessung. 


\subsubsection{Datenaufzeichnung und Auswertung}

Zur Aufzeichnung und Auswertung der Messdaten wurde ein eigens dafür entwickeltes Messsystem verwendet. Dieses sogenannte „Modular Intensive Care Data Acquisition System“ (MIDAS) wurde in Zusammenarbeit mit dem Institut für biomedizinische Technik der Hochschule Mannheim entwickelt und gestattet eine parallele, zeitsynchrone und kontinuierliche Erfassung, Digitalisierung, Visualisierung und Speicherung aller Messwerte. Die Daten wurden über MIDAS erfasst, verarbeitet und über USB 2.0 an einen Standard-Laptop übertragen. Die Echtzeitkurven wurden im Stream-To-DiskVerfahren binär auf der Festplatte gespeichert. Die Speicherung der Daten erfolgte in einer Access Datenbank (Microsoft Corporation, Redmond, USA).

\subsection{Durchführung der Versuche}

Nach Abschluss aller vorbereitenden Maßnahmen wurden die Tiere in Bauchlage gebracht. Sobald alle Geräte korrekt kalibriert waren, wurde mit der Aufzeichnung der Ausgangswerte begonnen und die Tiere wurden mit Hilfe der speziellen Transportvorrichtung unter kontinuierlicher Datenaufzeichnung und unterbrechungsfreier Beatmung zum Computertomographen gebracht.

$\mathrm{Zu}$ diesem Zeitpunkt musste die Randomisierung der Tiere zur jeweiligen Versuchsgruppe erfolgen: 1. Kontrollgruppe (Control), 2. Acute Intracranial Hypertension (AICH), 3. Acute Respiratory Distress Syndrome (ARDS), 4. ARDS + AICH.

Vor Versuchsbeginn wurde in allen 4 Gruppen nach Abschluss der Präparation und der initialen Datenaufzeichnung ein Ausgangsschädel- und Thorax-CT durchgeführt. Insgesamt erhielten die Versuchstiere 4 cerebrale und 4 thorakale CT-Untersuchungen an den Messpunkten $\mathrm{t}=0, \mathrm{t}=60, \mathrm{t}=120$ und $\mathrm{t}=240$ Minuten. Die Gesamtversuchsdauer wurde ebenfalls mit 240 Minuten angesetzt. Zu den oben genannten Messzeitpunkten wurden arterielle Blutproben entnommen. Das arterielle Blut wurde aus der rechten Arteria femoralis entnommen. Um eine Verdünnung durch die im Katheter befindliche Kochsalzlösung zu verhindern, wurden $5 \mathrm{ml}$ Blut vorgezogen. Das vorgezogene Eigenblut wurde dem Tier umgehend zurückgegeben. 


\section{5 Übersicht über die Versuchsgruppen}

Tabelle 3: Übersicht über die Versuchsgruppen

Gruppe

$\mathrm{AICH} \quad 7$

ARDS 7

$\mathrm{AICH}+\mathrm{ARDS}$

\section{n}

7

7

7

7

\section{Schaden}

\section{keiner}

intrakranielle Drucksteigerung $(\mathrm{AICH})$

akutes Lungenversagen (ARDS)

Kombination von $\mathrm{AICH}$ und ARDS

Tabellarische Darstellung der 4 Versuchsgruppen.

\subsubsection{Gruppe 1 - Kontrollgruppe (Control)}

In der Kontrollgruppe erfolgte keine Intervention, jedoch eine „Instrumentierung“. Es wurde das gleiche Zeitintervall eingehalten, um die Daten vergleichen zu können.

\subsubsection{Gruppe 2 - Acute Intracranial Hypertension (AICH)}

Der Begriff „Schädel-Hirn-Trauma“ ist weit gefasst und für einen wissenschaftlichen Versuch schwer zu definieren. Aufgrund der möglichen Vielfalt der Entstehung eines Schädel-Hirn-Traumas war es nötig, sich für einen reproduzierbaren und klinisch relevanten Schädigungsmechanismus zu entscheiden. In Anbetracht dessen kam am ehesten ein intrakranielles Druckmodell (acute intracranial hypertension) und kein klassisches SHT-Modell (fluid percussion) in Frage. Im Folgenden wird der Begriff „acute intracranial hypertension“ (AICH) daher anstelle des Begriffs „Schädel-Hirn-Trauma“ verwendet, um den genauen Schädigungsmechanismus besser zu veranschaulichen. Über ein separates Bohrloch $25 \mathrm{~mm}$ lateral des rechts frontalen Cortex wurde ein $22 \mathrm{~F}$ Fogarty Ballonkatheter (Edward Lifescience, LLC, Irvine, California, USA) eingelegt. Der Ballon wurde bis zu einem ICP von $>30 \mathrm{~cm} \mathrm{H}_{2} \mathrm{O}$ aufgeblasen (siehe Abbildung 14). 

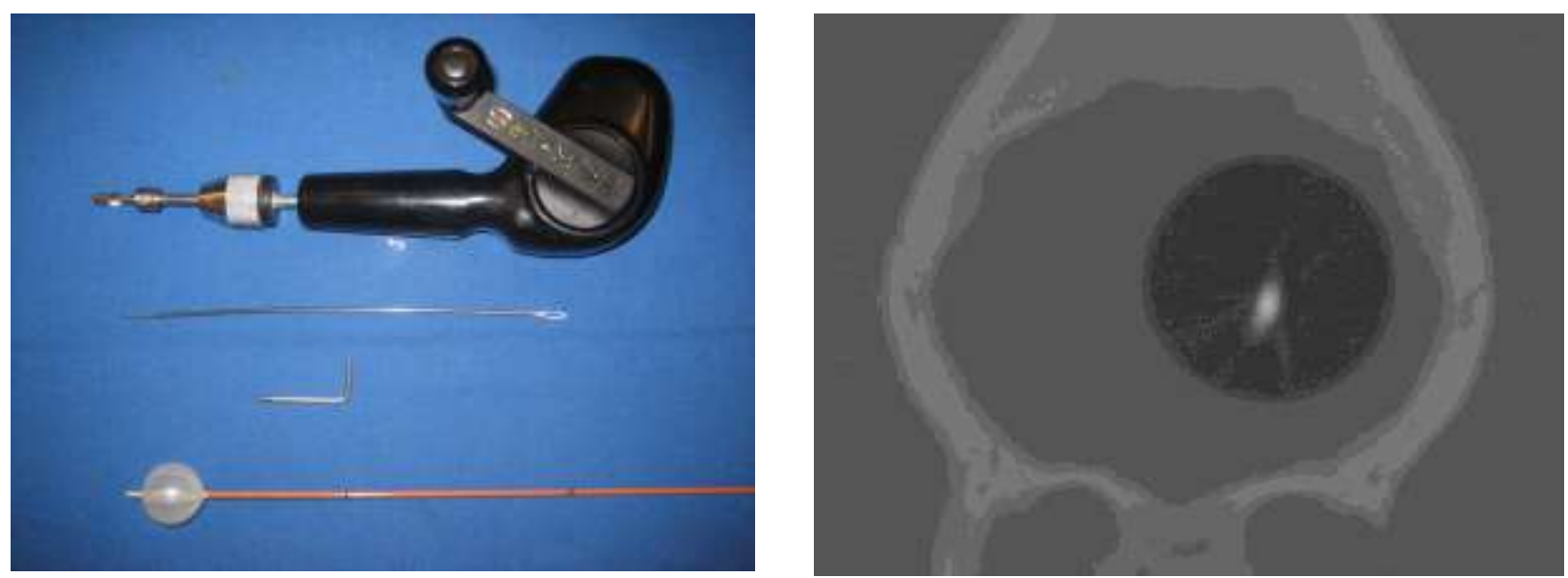

Links sind Bohrer, Sonde und Ballonkatheter zu sehen. Sie werden für die Etablierung des Hirndruckmodells benötigt. Rechts ist ein Schweinegehirn nach Balloninflation zu erkennen.

\subsubsection{Gruppe 3 - Acute Respiratory Distress Syndrome (ARDS)}

Der Lungenschaden (siehe Abbildung 15) wurde durch Injektion von Ölsäure über einen Zeitraum von 15 Minuten in den rechten Vorhof $(0,1 \mathrm{ml} / \mathrm{kg}$ in $20 \mathrm{ml}$ warmem $0,9 \% \mathrm{NaCl})$ induziert. Die Lungenschädigung wurde als konstant betrachtet, wenn nach 60 Minuten ein konstanter $\mathrm{PaO}_{2}$ von $<200 \mathrm{mmHg}$ bei einer $\mathrm{FiO}_{2}$ von 1,0 herrschte. Somit waren die Kriterien der Konsensus-Konferenz für ein ARDS erfüllt (Bernard et al. 1994).

Abbildung 15: links - gesunde Lunge ( $\left.T_{0}\right)$; rechts - Lunge nach Schädigung durch Ölsäure $\left(T_{240}\right)$
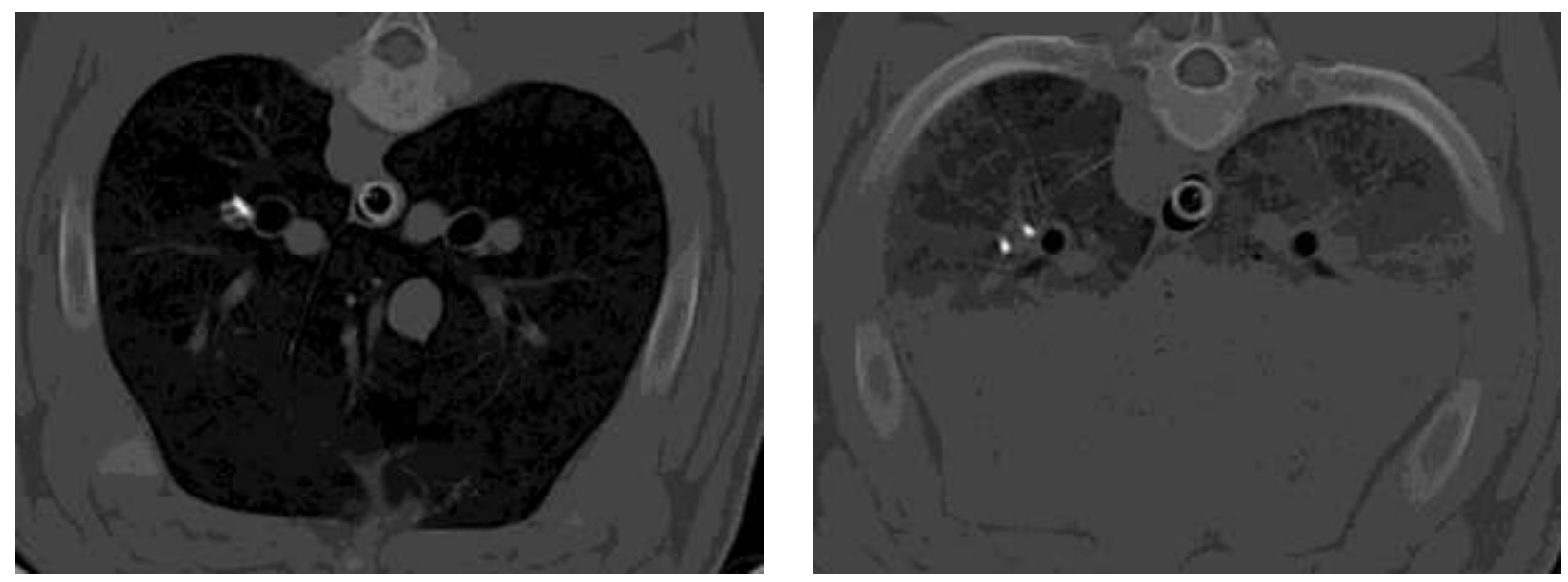

Die linke Abbildung zeigt eine gesunde Schweinelunge zum Messpunkt $T_{0}$. Rechts sieht man dieselbe Lunge zum Messpunkt $T_{240}$ nach Schädigung durch Ölsäure. 


\subsubsection{Gruppe 4 - ARDS + AICH}

Aufgrund der längeren Vorlaufzeit wurde zunächst der Lungenschaden mittels Ölsäure induziert. Nach dessen Etablierung erfolgte die Platzierung des Fogarty-Katheters und Induktion einer intrakraniellen Druckerhöhung mit einem ICP > $30 \mathrm{mmHg}$.

\section{6 Übersicht über die Messpunkte}

In allen Gruppen wurden an den folgenden Punkten Messwerte erhoben:

Tabelle 4: Übersicht über die Messpunkte

Messpunkt

$\mathbf{T}_{0}$

$\mathrm{T}_{60}$

$T_{120}$

$\mathbf{T}_{240}$
Zeit

Ausgang

60 Minuten

120 Minuten

240 Minuten

Die Tabelle zeigt eine chronologische Auflistung der festen Messpunkte.

\subsection{Versuchsende und Organ-/Probenentnahme}

Nach Durchführung aller Messpunkte wurden die Tiere wieder in den Tier-OP 2 verbracht. Um eine genau Beurteilung von möglichen pathologischen Veränderungen am Lungenparenchym zu ermöglichen, war es nötig, die Lunge mit Paraformaldehyd zu konservieren. Da die Perfusion nach Organentnahme mit einer großen Verzögerung von der Entnahme bis zur erfolgten Perfusion verbunden gewesen wäre, wurden alle Organe in situ perfundiert. Hierzu wurde das Tier nach Abschluss aller Messpunkte wieder in Rückenlage gebracht, die Narkose zunächst durch Bolusinjektion von Ketamin (3 mg/kg KG) sowie Midazolam (2 mg/kg KG) vertieft und anschließend eine Längsschnittthorakotomie durchgeführt. Nach Eröffnung des Thorax sowie des Pericards wurden die herznahen Gefäße freipräpariert (siehe Abbildung 16). 
Abbildung 16: Längsschnittthorakotomie und eröffnetes Perikard
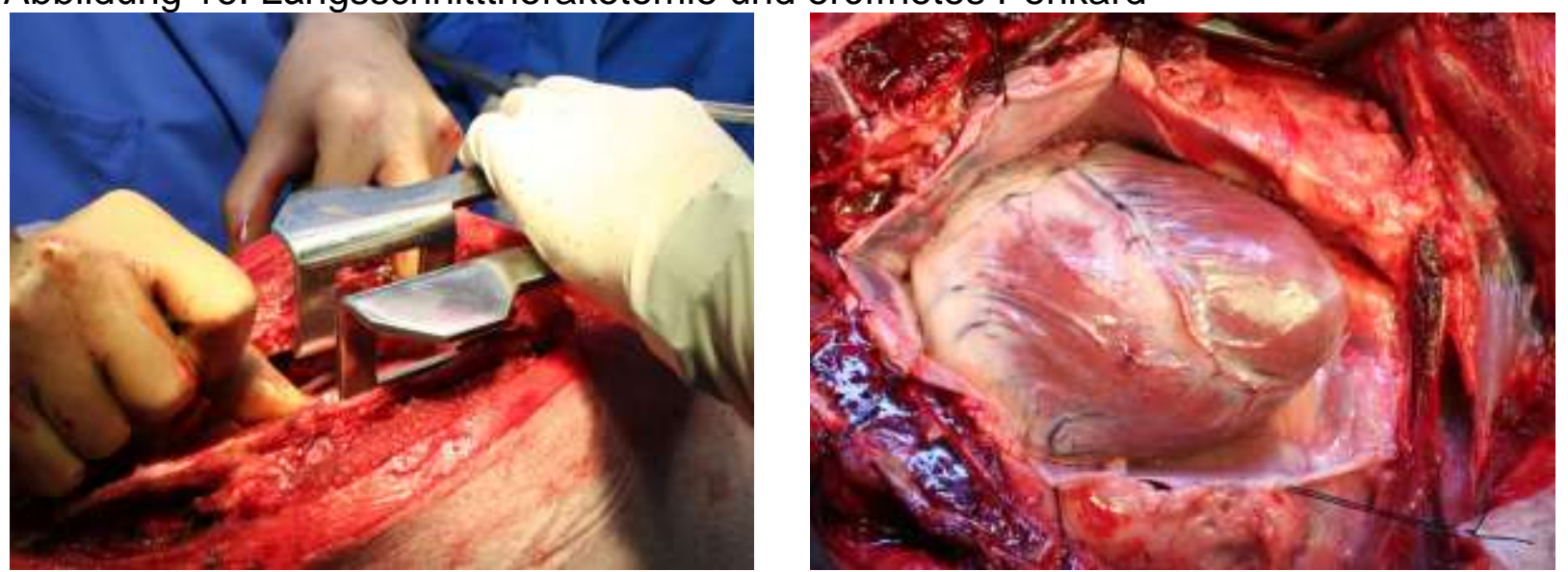

Links ist die Eröffnung des Thorax mit Hilfe eines Rippenspreizers zu sehen. Die rechte Abbildung zeigt das fertig präparierte, schlagende Herz mit bereits eröffnetem Perikardbeutel.

Nachdem die Gefäße gut sichtbar freipräpariert waren, wurde eine Tabaksbeutelnaht auf das rechte Atrium gesetzt. Das Atrium wurde mit einer Vorhofklemme fixiert und eine ca. 0,8 cm lange Inzision gemacht. Danach konnte die Perfusionskanüle in das rechte Atrium platziert und mit Hilfe der gesetzten Naht sicher fixiert werden (siehe Abbildung 17).

Abbildung 17: Tabaksbeutelnaht und Perfusionskanüle im rechten Vorhof
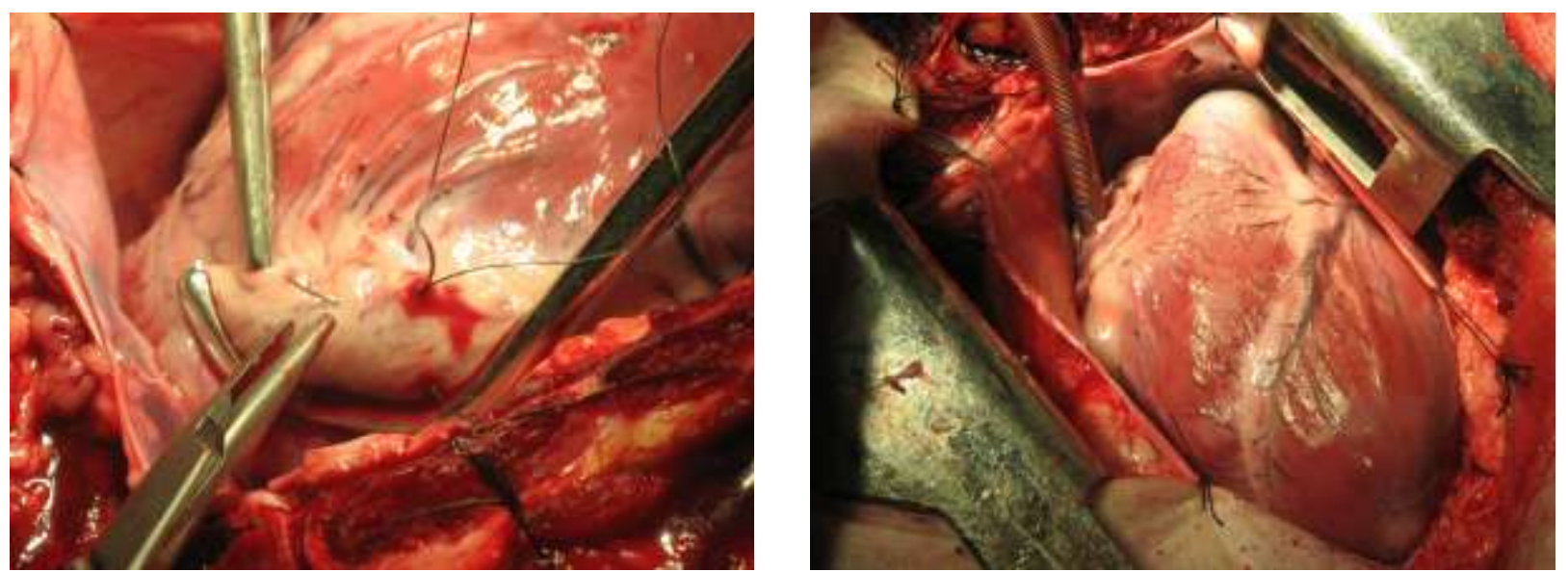

Links sieht man die Platzierung einer Tabaksbeutelnaht auf das Ohr des rechten Vorhofs. Die rechte Abbildung zeigt das freipräparierte Herz sowie die bereits im rechten Vorhof platzierte und mit Hilfe der Tabaksbeutelnaht fixierte Perfusionskanüle. 
Die zweite Kanüle wurde ca. 2-3 cm oberhalb der Aortenklappenebene platziert. Hierzu wurden ebenfalls eine Tabaksbeutelnaht sowie eine ca. 0,5 cm lange Inzision gesetzt (siehe Abbildung 18).

Abbildung 18: Inzision der Aorta und Platzierung der Perfusionskanüle
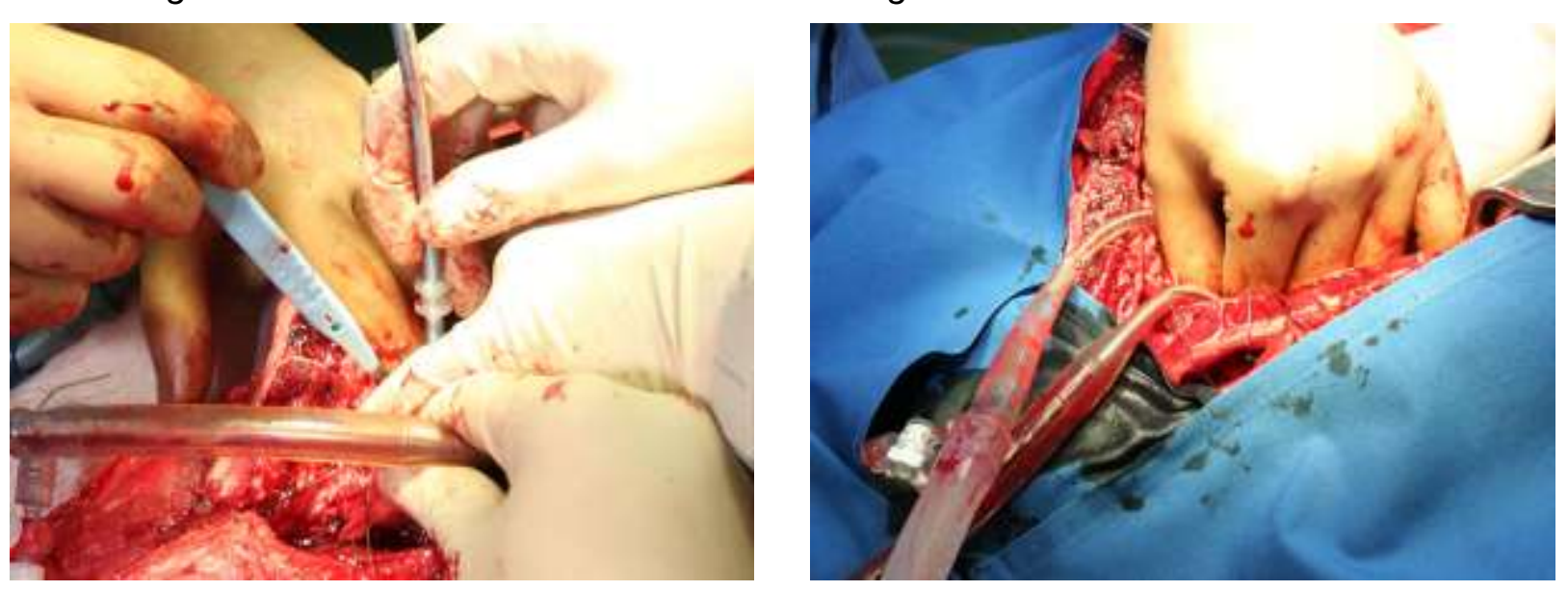

Links ist die unmittelbare Inzision der Aorta ascendens kurz oberhalb der Klappenebene zu erkennen. Rechts erkennt man die fertig platzierten Perfusionskanülen.

Die Perfusion mit einer Kreiselpumpe erfolgte anfangs bei schlagendem Herzen mit 20 I physiologischer Kochsalzlösung zwecks Exsanguination. Im Anschluss daran wurde der gesamte Organismus mit Paraformaldehyd-Lösung (PFA, Roth Karlsruhe, Deutschland) perfundiert. Die Entnahme der Lunge erfolgte in toto. 


\subsection{CT-Diagnostik}

Alle Röntgenuntersuchungen der Lunge wurden mit Hilfe eines GE Lightspeed V Computertomographen (GE Medical Systems, Wisconsin, USA) durchgeführt. Die Scans erfolgten immer in Bauchlage mit folgenden Parametern: Schichtdicke $5 \mathrm{~mm}$, Intervall 0,5 mm, $100 \mathrm{MA}, 100 \mathrm{kV}$. Die gesamte Lunge wurde von kranial nach kaudal innerhalb von 14 bis 19 Sekunden in endexspiratorischer Lage mit einem PEEP von $5 \mathrm{cmH}_{2} \mathrm{O}$ gescannt. Die Ausgabe der Daten geschah als 16-bit DICOM-Bild (Digital Imaging and Communication in Medicine). Die Analyse und Auswertung der DICOM-Bilder erfolgte durch das Programm MALUNA 3 (Mannheimer Lungen-Analyse-Tool), entwickelt von Dr. P. Hermann, ZARI, Universitätsklinik Göttingen. Die Software basiert auf der graphischen Programmiersprache LabVIEW 8.5 und ImaqVision (National Instruments, Austin TX, USA) und erlaubt anspruchsvolle Lungenanalyseverfahren wie Segmentierung, Dichteverteilung und vieles mehr.

Die ersten CT-Bilder von Patienten mit akutem Lungenversagen, heute ARDS, stammen aus den 80-er Jahren. Die Technik der Computertomographie war jedoch bereits zehn Jahre zuvor in der Klinik verfügbar. Es stellt sich die Frage, warum es so lange gedauert hat, bis diese Technik auch in der Diagnostik und Therapie des ARDS eingesetzt wurde. Zum einen waren die damaligen Apparate, verglichen mit den heutigen, extrem langsam und nicht sehr verbreitet. Die wenigen Geräte, die zur Verfügung standen, waren häufig ausgebucht und der Transport und die Lagerung von Intensivpatienten gestalteten sich ebenfalls schwierig. Zum anderen war man damals der Auffassung, dass es sich bei einem ARDS um einen homogen in der Lunge stattfindenden Prozess handelt, weshalb konventionelle, zweidimensionale Röntgenaufnahmen als ausreichend betrachtet wurden. Die CT-Diagnostik gab erstmals detaillierteren Aufschluss über die regionale Verteilung des ARDS und dessen Einfluss auf unterschiedliche Lungenbereiche (Gattinoni et al. 1986 b, Maunder et al. 1986). Mittlerweile gehört die Computertomographie zu einem der Standardverfahren in der Diagnostik und Therapieüberwachung des ARDS.

Mit der CT-Untersuchung gelang es, die verschiedenen Morphologien des ARDS näher zu beschreiben, und man fand heraus, dass es sich physikalisch um einen vertikalen Dichtegradienten handelt, der von ventral nach dorsal zunimmt (Hedlund et al. 1982, Hedlund et al. 1985). Mit Hilfe einer quantitativen Analyse der Lungendichte kann man die pathophysiologischen Mechanismen näher betrachten und verstehen. Diese Untersuchungsmethode basiert auf der Abhängigkeit einer Abschwächung von Röntgenstrah- 
lung in einem definierten Volumen (die Maßeinheit für dieses Volumen im CT ist ein Voxel) und der physikalischen Dichte des betreffenden Lungevolumens, gemessen in Hounsfield Units (HU) (Mull 1984).

Zum Verständnis ist es sinnvoll, sich den menschlichen Körper als ein Objekt aus transversalen Schichten vorzustellen. Jede einzelne dieser Schichten hat wiederum eine definierte Dicke, die sich aus quaderförmigen Elementen zusammensetzt. Jedes einzelne dieser Elemente repräsentiert einen Zahlenwert. Diese Elemente bezeichnet man auch als Voxel. Das Wort Voxel setzt sich aus den Begriffen „volumetric“ und „pixel“ zusammen. Hierbei handelt es sich um ein dreidimensionales „Pixelmodell“, das mit Hilfe von XYZ-Koordinaten die Position einzelner Pixel im Raum definieren und deren Grauwert als Dichtemaß wiedergeben kann (siehe Abbildung 19). Diese diskreten Werte können dann als Dichte interpretiert und den entsprechenden Geweben (Knochen, Fett etc.) zugeordnet werden. Die Computertomographie misst einen linearen Schwächungskoeffizienten. Dieser Schwächungskoeffizient wird zur Veranschaulichung relativ zur Schwächung von Wasser angegeben und nach seinem Erfinder in Hounsfield Units (HU) angegeben (Hounsfield 1973).

\section{Abbildung 19: Voxelmodell}

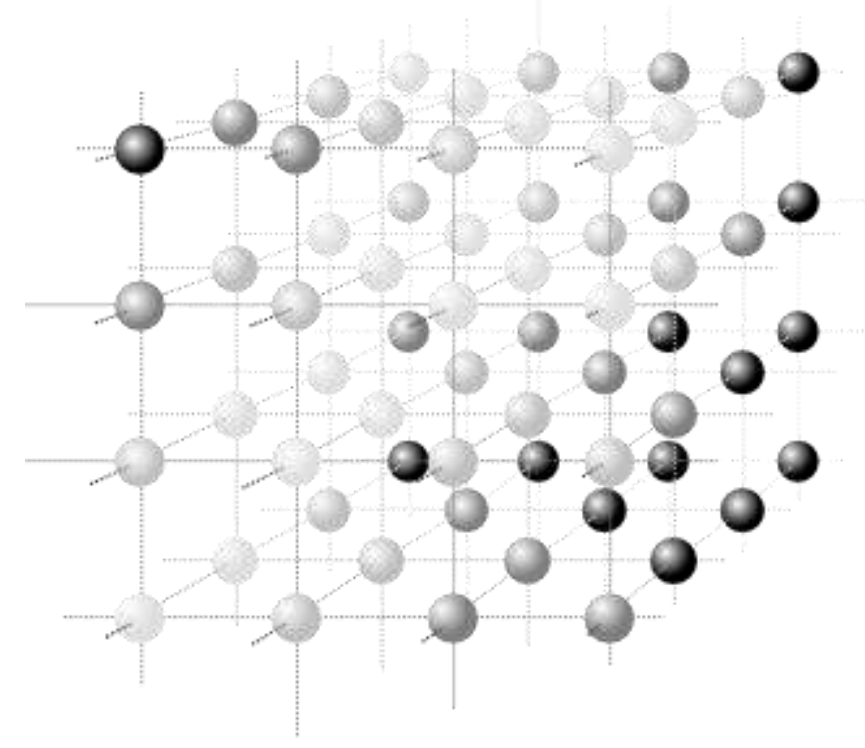

Schematische Darstellung des Voxelmodells als dreidimensionales Gitternetz.

Wie bei jedem Röntgenverfahren werden umso weniger Elektronen detektiert, je größer die Abschwächung der Röntgenstrahlen im durchdrungenen Gewebe ist. Die Hounsfield Units können über die Abschwächung von Röntgenstrahlen in jedem beliebigen Voxel 
des entsprechenden Gewebes bestimmt werden. Die Skala der Abschwächung von Röntgenstrahlen wurde willkürlich festgesetzt. So hat Knochen einen Wert von +1000 HU (komplette Absorption), Luft einen Wert von -1000 HU (keine Absorption) und Wasser einen Wert von 0 HU zugeordnet bekommen. Ein Voxel von -1000 HU besteht somit allein aus Gas, ein Voxel von $0 \mathrm{HU}$ aus Wasser und ein Voxel von $-500 \mathrm{HU}$ aus ca. $50 \%$ Wasser und 50\% Gas. Basierend auf folgender Formel:

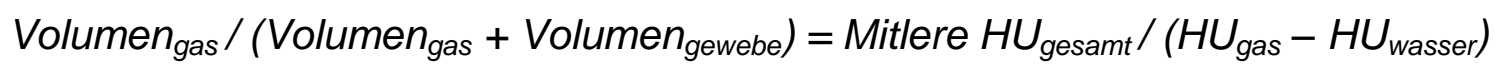

lässt sich durch Umstellung eben dieser für jeden beliebigen Bereich der Lunge, dessen Volumen bekannt ist, das Volumen von Gas, das Volumen von Gewebe und das Verhältnis von Gas zu Gewebe bestimmen (Gattinoni et al. 1986 a). In einer gesunden Lunge wird die Dichte im CT von verschiedenen Komponenten bestimmt: der Anteil an Gas (-1000 HU), der Anteil an Gewebe und Blut (20-40 HU) und der Anteil an Wasser (0 HU). Da ein Voxel eine fest definierte Größe besitzt, variiert die Genauigkeit der Differenzierung eines bestimmten Gewebes mit der Größe des Voxel. Ältere CT-Geräte hatten eine schlechte Ortsauflösung mit großen Schichtdicken und großen Voxeln. Schließt ein Voxel nun hypothetisch mehrere anatomische Strukturen der Lunge ein, so kann deren Dichteänderung entweder auf einer Abnahme von Gas oder einer Zunahme von Gewebe basieren. Das Voxel ist nicht in der Lage, dies zu unterscheiden. Mittlerweile sind Computertomographen in der Lage, sehr dünne Schichten von z.B. 0,5 mm zu fahren. Die Voxel werden dadurch im Verhältnis kleiner und die räumliche Ortsauflösung verbessert. Dies führt zu einer zuverlässigeren Bestimmung von Dichtewerten einzelner anatomischer Strukturen.

Kennt man nun die gesamten Voxel und deren Dichtewerte eines Lungenbereichs oder gar einer ganzen Lunge, so lässt sich die Lunge hiermit in verschiedene Kompartimente einteilen. Üblicherweise reicht diese Einteilung über 11 Kompartimente von -1000 HU bis $+100 \mathrm{HU}$ (siehe Abbildung 20). Die Anzahl bestimmter Voxel in einem Kompartiment lässt sich dann prozentual, bezogen auf die Gesamtzahl an gemessenen Voxeln, darstellen (Gattinoni et al. 1986 a, Gattinoni et al. 1987). 
Abbildung 20: Lungenkompartimente nach Hounsfield Units eingeteilt

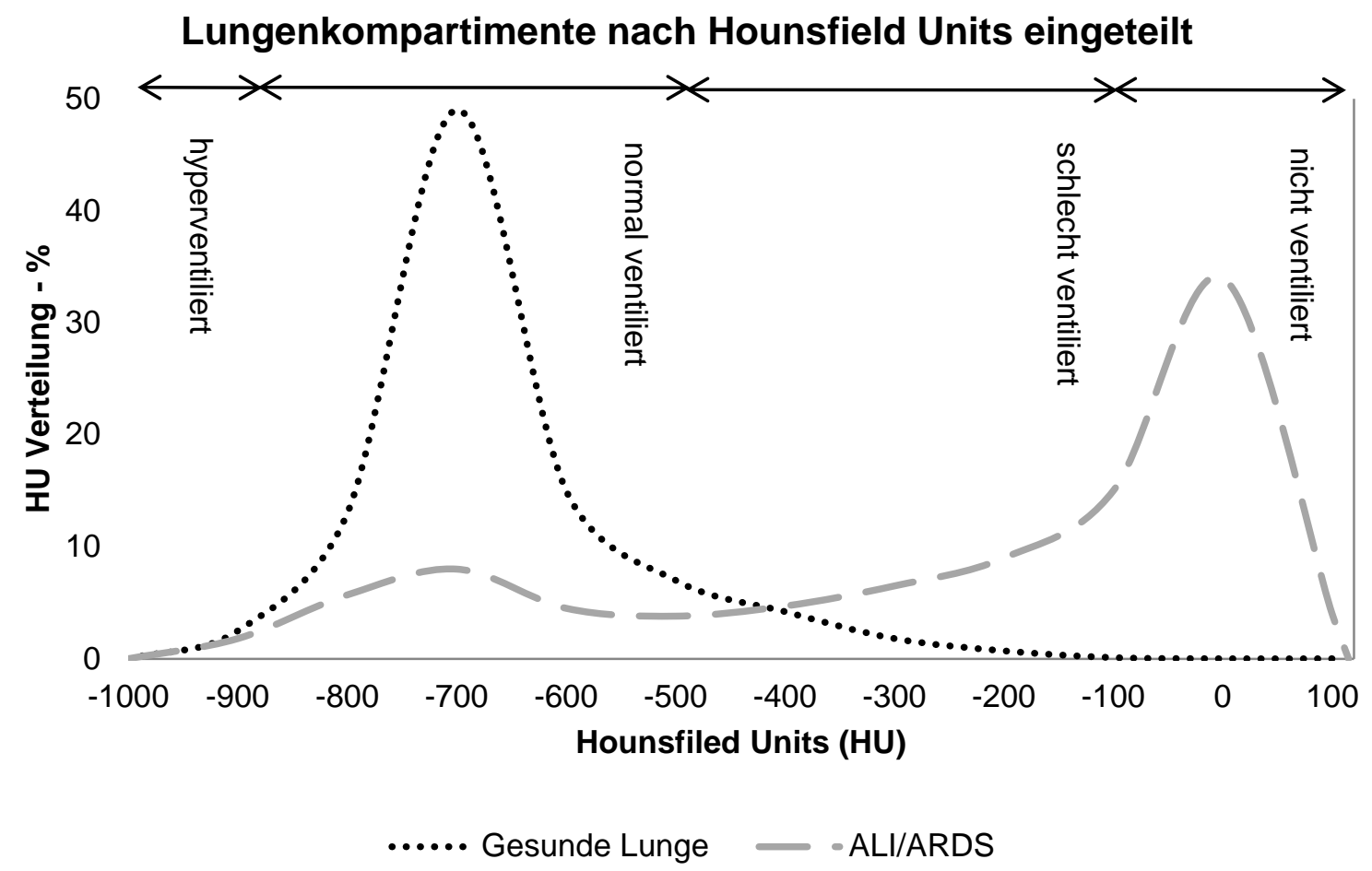

Die obige Abbildung stellt die Verteilung der Lungenparenchymdichte in gesunden und kranken Lungen anhand einer prozentualen Verteilung der Hounsfield Units dar.

Wie in Abbildung 2 zu sehen ist, befinden sich bei einer gesunden Lunge (Ausgangsposition) ca. $50 \%$ der Voxel in einem Bereich zwischen $-600 \mathrm{HU}$ bis $-800 \mathrm{HU}$, was im Schnitt ungefähr 70\% Gas und 30\% Gewebe entspricht. Bei forcierter Inspiration oder Beatmung mit PEEP (totale Lungenkapazität) würden sich etwa $20 \%$ der Kompartimente in den Bereich zwischen $-900 \mathrm{HU}$ bis $-1000 \mathrm{HU}$ verschieben (i.e. $85 \%$ Gas und $15 \%$ Gewebe) (Vieira et al. 1998). Mit Hilfe der oben genannten Formel und der ermittelten HU kann man so, bei bekanntem Volumen, den Gewebeanteil eines Lungenbereichs bestimmen. Wichtig zu erwähnen ist, dass es sich bei Gewebe nicht ausschließlich um Lungengewebe handeln muss, sondern auch Blut, extrazelluläre Matrix und Zellschrott etc. (i.e. alle Strukturen mit einer Dichte um $0 \mathrm{HU}$ ) mit einfließen.

Es ist also möglich, anhand der Voxel zwischen gut belüfteten und weniger gut belüfteten Bereichen der Lunge zu unterscheiden (Gattinoni et al. 1987). Die meisten Autoren haben hier zwischen vier Bereichen differenziert: 1. überblähte Lungenbereiche, 2. normal belüftete Bereiche, 3. schlecht belüftete Bereiche und 4. nicht belüftete Bereiche. Die Festsetzung dieser Bereiche anhand der HU variiert leicht je nach Autor. Im Folgenden haben wir uns an den Werten von Gattinoni et al. orientiert (siehe Tabelle 5). 
Tabelle 5: Hounsfield-Einteilung verschiedener Lungenbereiche nach Gattinoni

Nicht belüftet Schlecht belüftet Normal belüftet

Überbläht

Gattinoni et al.

+100 bis $-100 \mathrm{HU}$

-100 bis $-500 \mathrm{HU}$

-500 bis $-900 \mathrm{HU}$

-900 bis $-1000 \mathrm{HU}$

Tabellarische Darstellung der Einteilung von nicht, schlecht, normal belüfteten und überblähten Lungenbereichen anhand von Hounsfield Units (Gattinoni et al. 1987).

Entscheidende Informationsgewinne über die Pathophysiologie des ARDS ergaben sich aus der regionalen Verteilungsanalyse von CT-Bildern der Lunge (siehe Abbildung 23). Hierzu wurde eine Einteilung des axialen CT-Bildes der Lunge in 10 gleich breite Abschnitte vom Sternum zur Wirbelsäule vorgenommen. In jedem dieser 10 Intervalle wird dann die gleiche Analyse durchgeführt, wie sie für die gesamte Lunge beschrieben wurde; damit wird wiederum die Bestimmung des Gas/Gewebe-Verhältnisses für die einzelnen Segmente möglich (Pelosi et al. 1994). Dieses Verfahren erlaubt z.B. einen Vergleich zwischen abhängigen und nicht abhängigen Lungenarealen und deren unterschiedliche Beeinflussung durch ein ARDS.

In dieser Arbeit wurde die äußere Begrenzung der Lunge an der Innenseite des Thorax und die innere Begrenzung entlang der Organe des Mediastinums für jedes einzelne CT-Bild per Hand umrandet. Alle Strukturen, die nicht zur Lunge gehörten, wurden von der Markierung ausgeschlossen. Die Software analysiert daraufhin anhand der markierten ROI (region of interest) die gesamte Lunge quantitativ (Rylander et al. 2005) und bestimmt die Dichte in Hounsfield-Einheiten, den Gasgehalt sowie den prozentualen Anteil an normal, schlecht und nicht belüfteten Lungenarealen. Zusätzlich lassen sich verschiedene Segmentierungsanalysen durchführen (2, 3, 4 und 10 Segmente). Die so bestimmten Werte wurden dann einer regionalen Verteilungsanalyse unterzogen. Hierbei wurde das markierte Lungengewebe von nichtabhängigen zu abhängigen Arealen entlang der Vertikalen unterteilt (siehe Abbildungen 21 und 22). 
Abbildung 21: Schematische Segmenteinteilung der Lunge vor Schädigung

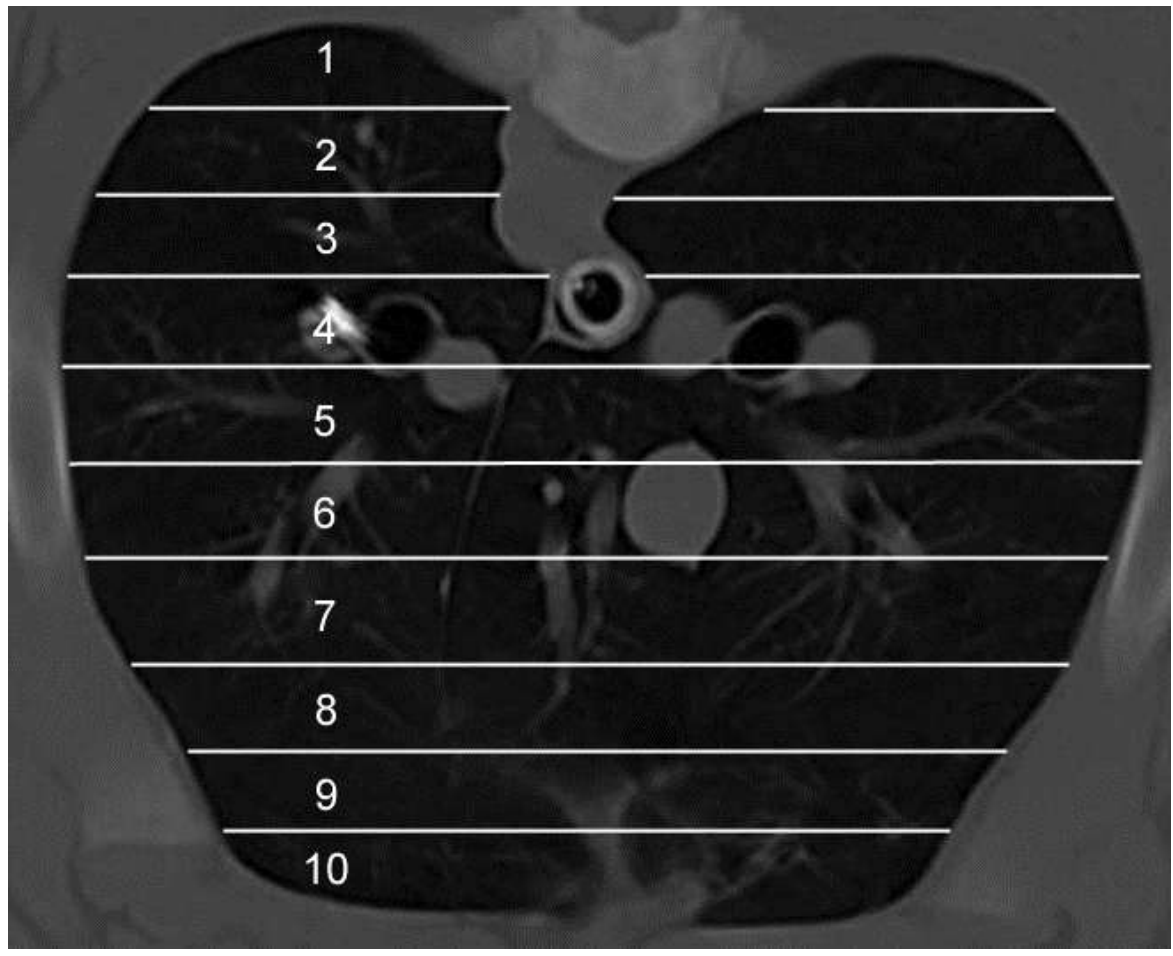

Dorso-ventrale Einteilung einer gesunden Schweinelunge ( $\left.T_{0}\right)$ in zehn Segmente entlang der Vertikalen.

Abbildung 22: Schematische Segmenteinteilung der Lunge nach Schädigung

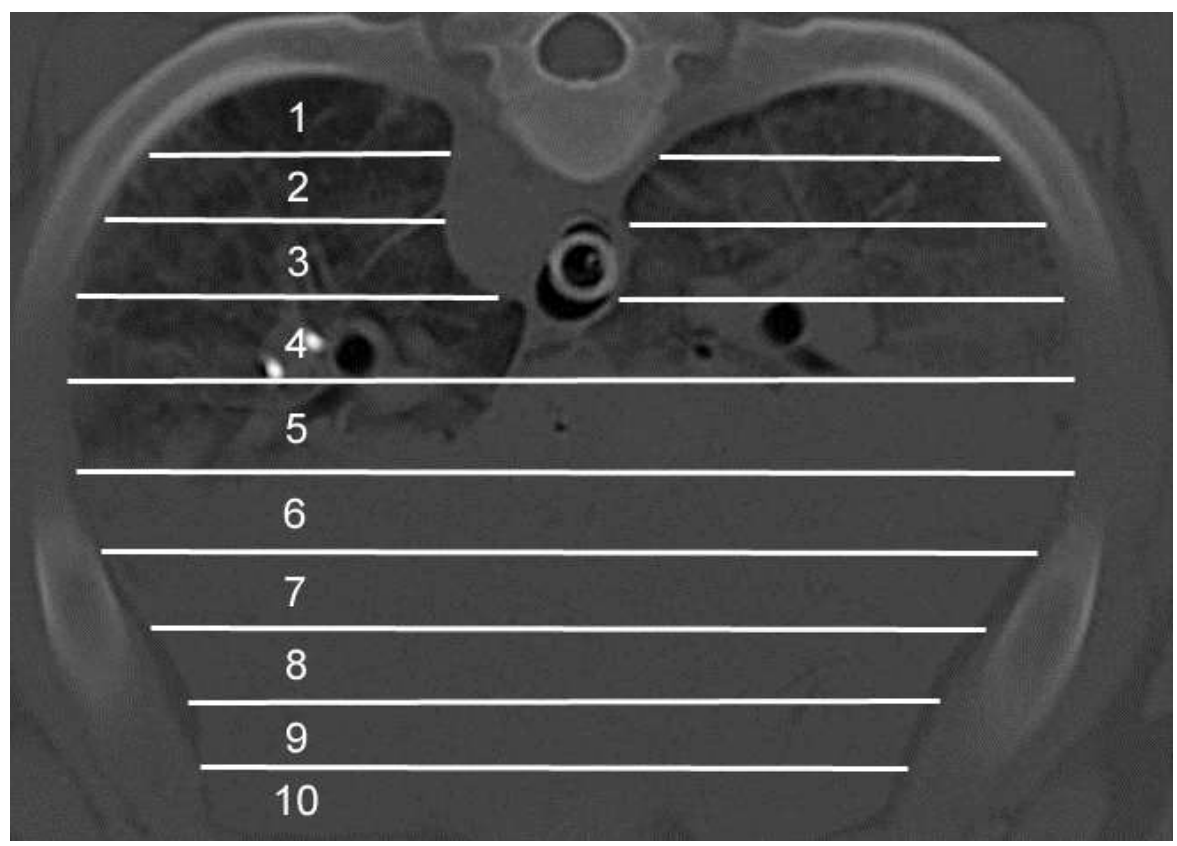

Dorso-ventrale Einteilung einer geschädigten Schweinelunge ( $\left.T_{240}\right)$ in zehn Segmente entlang der Vertikalen. 


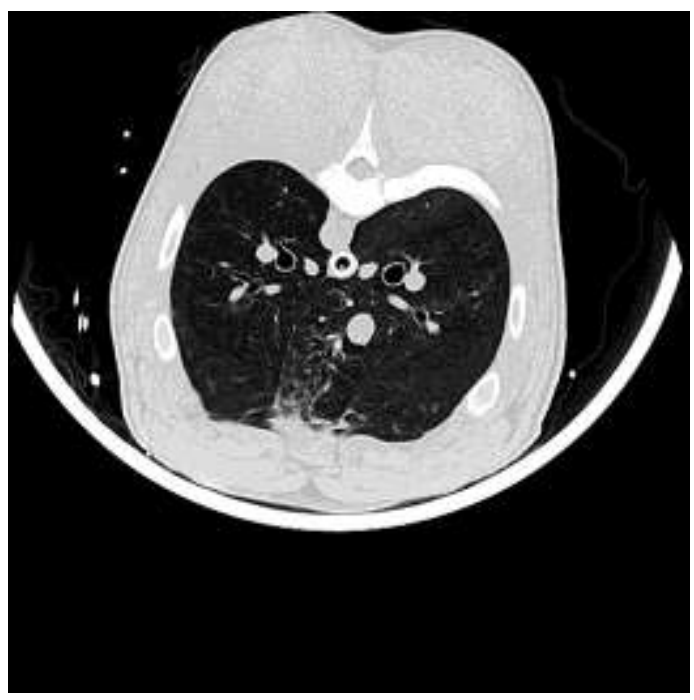

Control

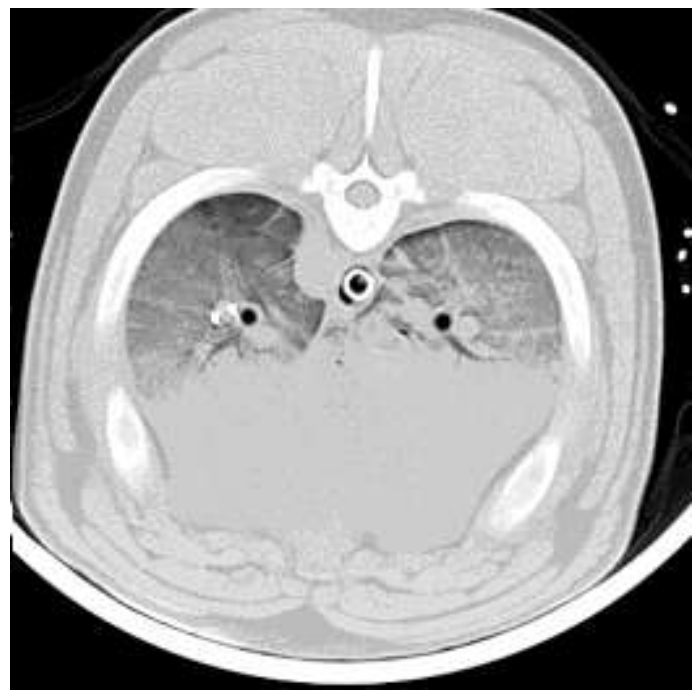

ARDS

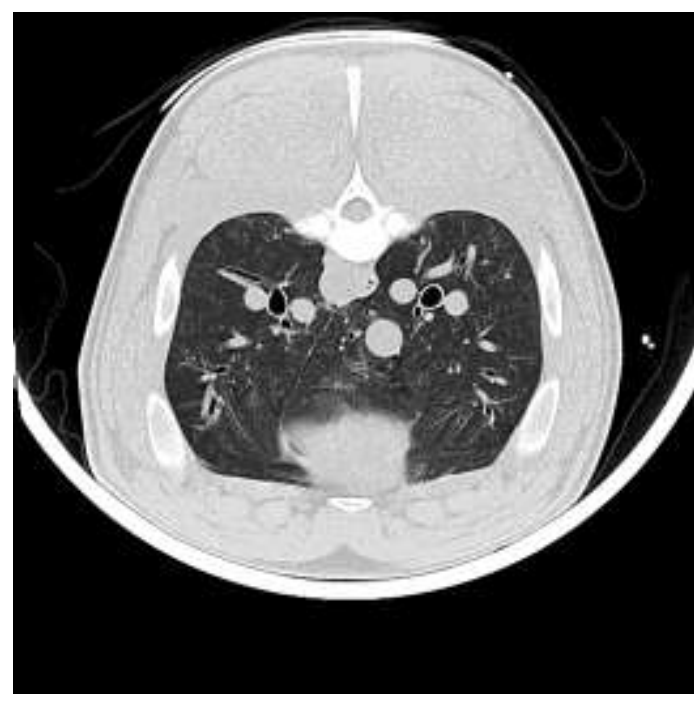

$\mathrm{AICH}$

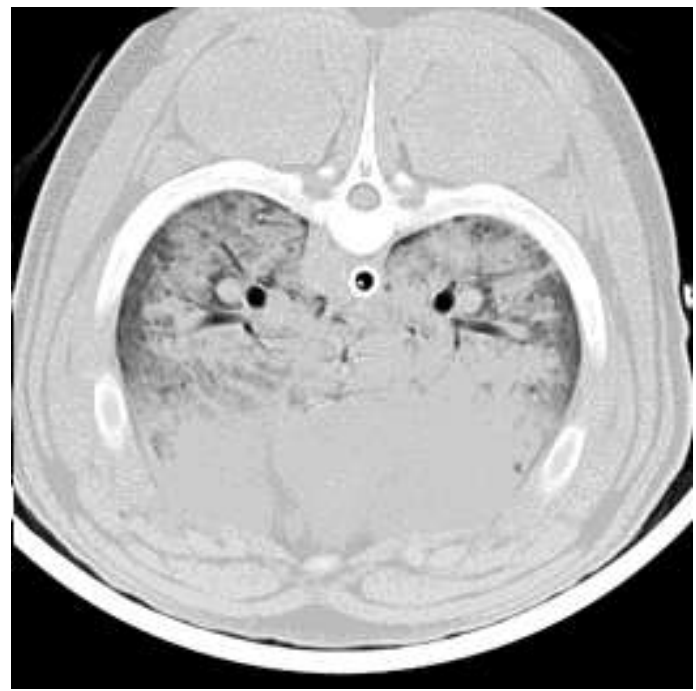

$\mathrm{ARDS}+\mathrm{AICH}$

Die vier Abbildungen zeigen jeweils ein Lungenschnittbild aus den vier Versuchsgruppen zum Messpunkt $T_{240}$. (Publiziert in Intensive Care Med (2011) 37:11821191, Fig.1)

Lungengewebe mit einer Dichte zwischen -1000 bis -900 HU wurde als überbläht eingeteilt, -900 bis $-500 \mathrm{HU}$ als normal ventiliert, -500 bis $-100 \mathrm{HU}$ als schlecht belüftet und -100 bis $+100 \mathrm{HU}$ als nicht belüftet (atelektatisch).

Alle Formeln zur Berechnung des Lungen-Gas-Volumens basieren auf Puybasset et al. (Puybasset et al. 2000 a, Puybasset et al. 2000 b, Rouby et al. 2000). 


\subsection{Extravaskulärer Lungenwasserindex (EVLWI)}

Das extravaskuläre Lungenwasser (EVLW) umfasst die Flüssigkeitsmenge im Lungengewebe. Im klinischen Alltag hilft dieser Parameter bei der Steuerung des Flüssigkeitshaushaltes und kann zusätzlich als Marker für die Entwicklung eines Lungenödems genutzt werden. Davey-Quinn et al. zeigten in ihrer Studie, dass das extravaskuläre Lungenwasser gut mit dem Outcome von Patienten mit ARDS korreliert (Davey-Quinn et al. 1999). Kommt es z.B. im Rahmen eines ARDS zu einem vermehrten Übertritt von Flüssigkeit aus den Gefäßen ins Interstitium oder in die Alveolen, so steigt der Gehalt an Flüssigkeit im Lungengewebe (Sakka et al. 2002). Die klinische Untersuchung, eine Thoraxröntgenaufnahme oder Blutgasanalyse sind zwar in der Lage, große Flüssigkeitsmengen zu erfassen, können allerdings nur in begrenztem Maße als frühe Indikatoren für die Entwicklung eines Lungenödems genutzt werden (Boussat et al. 2002, Halperin et al. 1985).

Mittlerweile gibt es verschiedene Techniken zur Bestimmung des extravaskulären Lungenwassers. Den Goldstandard stellt das COLD-Verfahren dar. COLD steht für Circulation, Oxygenierung, Lungenwasser und Diagnostik. Es ist ein Verfahren, welches auf der kombinierten Anwendung von Thermodilution und Farbstoffdilution beruht. In diesem Versuch wurde ausschließlich die weit verbreitete Thermodilutionsmethode angewandt. Hierzu sind ein zentralvenöser und ein spezieller arterieller Katheter mit Thermistor erforderlich. Über den zentralvenösen Katheter wird ein Bolus kalter, isotonischer Kochsalzlösung $\left(<8^{\circ} \mathrm{C}\right)$ injiziert, der dann das kardiopulmonale System passiert. Der Thermistor des arteriellen Katheters misst die Temperaturveränderung stromabwärts (siehe Abbildung 24). Aus dieser Messung lässt sich eine transpulmonale Thermodilutionskurve auftragen, aus deren Fläche mit Hilfe der Stewart-Hamilton-Gleichung das Herzzeitvolumen berechnet werden kann. Aus der mittleren Durchgangszeit (MTt $=\mathrm{Me}-$ an Transit Time) und Abfallzeit (DSt $=$ Down Slope Time) dieser Thermodilutionskurve können dann weitere Parameter wie z.B. das Vorlastvolumen oder das Lungenwasser bestimmt werden (siehe Abbildung 25). 
Abbildung 24: Thermodilutionsmethode - HZV (Pulsion 2002)

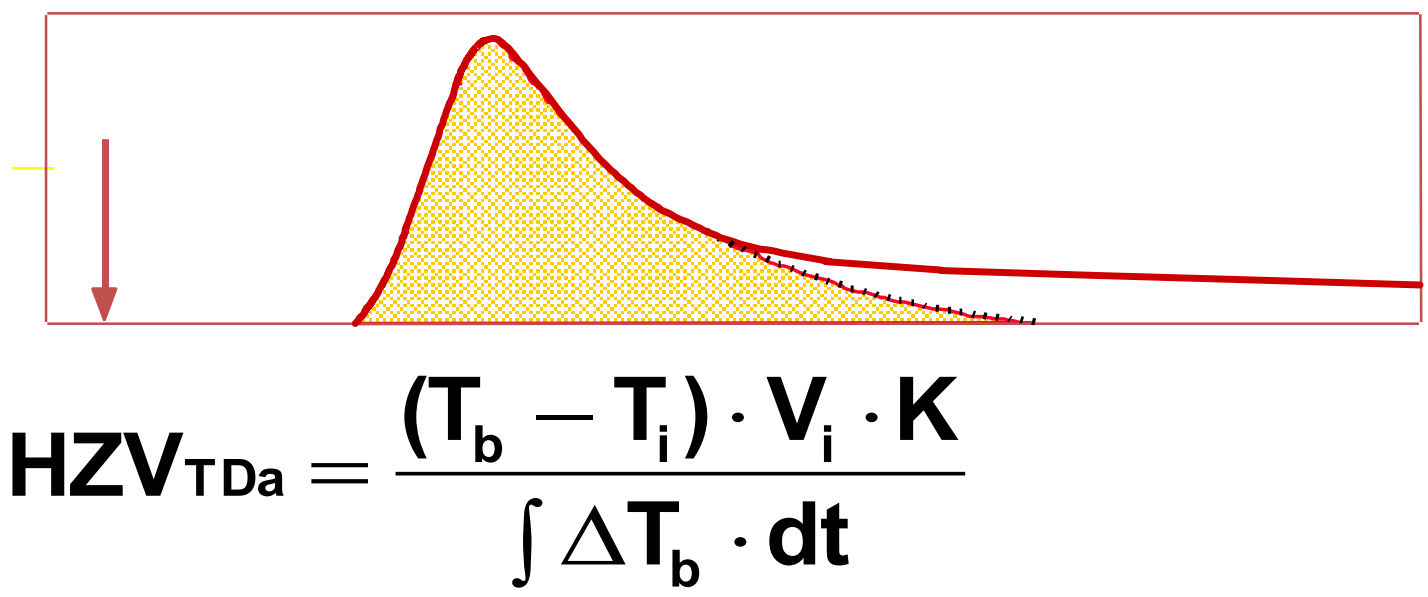

Oben ist der Verlauf einer Thermodilutionskurve schematisch dargestellt. Darunter findet sich die Stewart-Hamilton-Gleichung zur Berechnung des Herzzeitvolumens nach der Thermodilutionsmethode.

Abbildung 25: Thermodilution - Volumenparameter (Pulsion 2002)

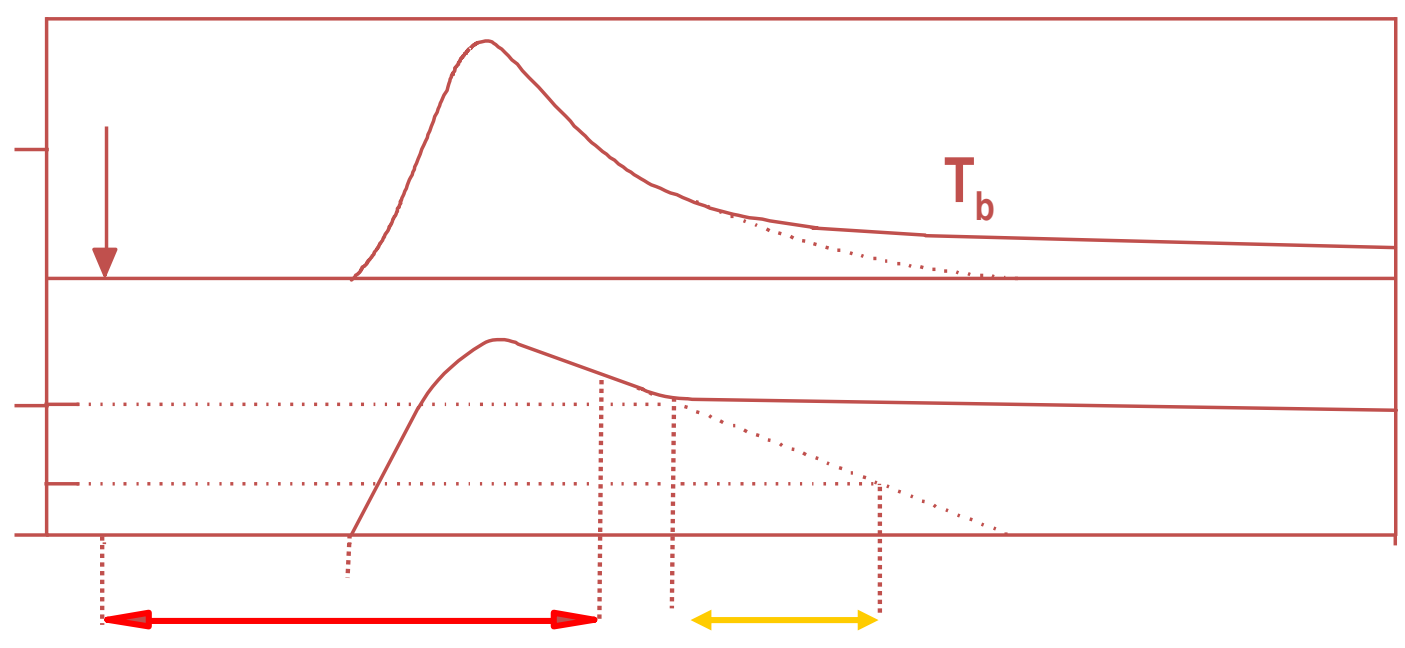

Schematische Darstellung der „Mean Transit Time“ (MTt) und der „Downslope Ti$m e^{\prime \prime}(D S t)$.

Nach einer Injektion passiert der Indikator, in diesem Fall kaltes Kochsalz, folgende anatomische Kompartimente: rechter Vorhof, rechter Ventrikel, pulmonale Gefäßstrombahn, linker Vorhof, linker Ventrikel. Diese anatomischen Kompartimente sind der Verteilungsraum für den injizierten Indikator, wobei die Lunge das größte Kompartiment darstellt. Um das extravaskuläre Lungenwasser bestimmen zu können, muss zunächst das intrathorakale Thermovolumen (ITTV) bestimmt werden. 


$$
I T T V=R A E D V+R V E D V+\text { Lungen }+ \text { LAEDV }+ \text { LVEDV }=\text { MTt } x \text { Fluss }(H Z V)
$$

Das größte Mischvolumen Kompartiment (Lunge, PTV = pulmonales Thermovolumen) erhält man durch Multiplikation der exponentiellen Abfallzeit (DSt) mit dem Herzzeitvolumen.

$$
\text { PTV }=\text { Pulmonales Thermovolumen }=D S t{ }^{*} \text { Fluss }(H Z V)
$$

Zur weiteren Berechnung des extravaskulären Lungenwassers ist es nun notwendig, das globale enddiastolische Volumen (GEDV), also die Blutmenge im Herzen am Ende der Diastole, zu kennen. Das globale enddiastolische Volumen errechnet sich wie folgt:

$$
\text { GEDV }=I T T V-P T V
$$

Der einzige Parameter, der nun zur Berechnung des extravaskulären Lungenwassers fehlt, ist das intrathorakale Blutvolumen (ITBV). Das intrathorakale Blutvolumen lässt sich mit einer Doppelindikatormethode, die hier nicht näher ausgeführt werden soll, direkt bestimmen. Sakka et al. haben in einer klinischen Studie herausgefunden, dass sich das globale enddiastolische Volumen um konstant $25 \%$ größer erweist als das mit Doppelindikatormethode bestimmte intrathorakale Blutvolumen (Sakka et al. 2000). Es ist also möglich, das intrathorakale Blutvolumen aus dem globalen enddiastolischen Volumen zu berechnen:

$$
\text { ITBV }=1,25 \times G E D V
$$

Das extravaskuläre Lungenwasser ermittelt sich nun aus der Differenz von intrathorakalem Thermovolumen und intrathorakalem Blutvolumen:

$$
E V L W=I T T V-I T B V
$$

Beim extravaskulären Lungenwasserindex (EVLWI) handelt es sich um das extravaskuläre Lungenwasser (EVLW) bezogen auf die Körperoberfläche. 


\subsection{Histologische Untersuchung der Lunge}

Die Gewebeproben wurden nach der in-situ-Perfusion und Entnahme des Organs gewonnen. Die Auswertung der Proben erfolgte durch zwei erfahrene Pathologen. Die Untersuchung geschah doppelblind.

Aus jeder Lunge wurden insgesamt 15 Gewebeproben entnommen, davon waren jeweils drei aus den drei rechten Lungenlappen und drei aus den zwei linken Lungenlappen. Es konnten also insgesamt 420 Proben bzw. 105 pro Gruppe analysiert werden. Nach Fixation in Paraformaldehyd erfolgte das Einbetten in Paraffin, und die Gewebestücke wurden in $5 \mu \mathrm{M}$ dünne Schichten geschnitten. Zur Färbung wurde Hematoxilineosin verwandt. Zur Betrachtung wurde ein Olympus BX41 Mikroskop eingesetzt.

Die Schnitte wurden mit Hilfe eines semiquantitativen Scoring-Systems (siehe Tabelle 6) analysiert, um das Ausmaß und den Schweregrad von Gewebsschädigung, Dystelektasen, pulmonalem Ödem und pulmonal arterieller Embolien sichtbar zu machen.

Tabelle 6: Histologisches Scoringsystem der Lunge

\begin{tabular}{|c|c|c|c|c|c|}
\hline Score & Ausmaß & Schweregrad & Dystelektasen & Ödem & Embolisation \\
\hline 0 & kein Schaden & kein Schaden & $\begin{array}{l}\text { keine Dystelekta- } \\
\text { sen }\end{array}$ & kein Ödem & kein Embolus \\
\hline 1 & $<10 \%$ der Fläche & $\begin{array}{l}\text { Hyperaemie + Gra- } \\
\text { nulozyten }\end{array}$ & $<10 \%$ der Fläche & $<10 \%$ der Fläche & In Subsegmentarterie \\
\hline 2 & $10-50 \%$ der Fläche & $\begin{array}{l}\text { + fibrinöses Ödem, } \\
\text { intraalveoläre Gra- } \\
\text { nulozyten + Blut }\end{array}$ & $\begin{array}{l}10-50 \% \text { der Flä- } \\
\text { che }\end{array}$ & $\begin{array}{l}10-50 \% \text { der Flä- } \\
\text { che }\end{array}$ & In Segmentarterie \\
\hline 3 & $50-75 \%$ der Fläche & + Nekrosen & $\begin{array}{l}50-75 \% \text { der Flä- } \\
\text { che }\end{array}$ & $\begin{array}{l}50-75 \% \text { der Flä- } \\
\text { che }\end{array}$ & In Lobusarterie \\
\hline 4 & $>75 \%$ der Fläche & --- & $>75 \%$ der Fläche & $>75 \%$ der Fläche & $\overline{---}$ \\
\hline
\end{tabular}

Die Tabelle zeigt das fünfstufige Scoring-System zur histologischen Einteilung der Lungenschädigung. 


\section{Ergebnisse}

\subsection{Statistische Auswertung}

Alle Werte wurden als Mittelwerte (+/- Standardabweichung) oder Mediane $(25 \%+75 \%$ Quartil) berechnet. Der Vergleich über den Versuchszeitraum erfolgte mit Hilfe des Friedmann- und des Wilcoxon-Tests. Zur Analyse der Gruppen untereinander wurde der Mann-Whitney-U-Test angewandt. Aufgrund der begrenzten Fallzahl wurden nichtparametrische Testverfahren verwendet. Ein $p$-Wert kleiner 0,05 wurde als signifikant betrachtet.

Alle Analysen wurden mit Hilfe der Software „Statistica für Windows 8.0“ (Statsoft, Europa) durchgeführt.

\subsection{Hämodynamik}

Die Werte für die Hämodynamik aller Gruppen vom Zeitpunkt Baseline $\left(T_{0}\right)$ bis $T_{240}$ können der Tabelle 7 entnommen werden. Abbildungen 26 und 27 stellen den zeitlichen Verlauf von Herzfrequenz und Schlagvolumen aller Gruppen dar. Abbildung 28 zeigt die Entwicklung des Herzzeitvolumens über 240 Minuten.

Zum Zeitpunkt $\mathrm{T}_{0}$ (Baseline) ergaben sich keine Unterschiede bei den hämodynamischen Parametern. Sowohl in der Kontrollgruppe als auch in der AICH-Gruppe waren die Werte konstant. Einzig das Herzzeitvolumen (HZV) war zu den Zeitpunkten $\mathrm{T}_{120}$ und $\mathrm{T}_{240}$ in der Gruppe AICH signifikant erhöht.

In den Gruppen ARDS und AICH + ARDS erhöhten sich die Werte für den mittleren pulmonalarteriellen Druck (MPAP) und die Herzfrequenz (HF) über die Zeit, wohingegen das Schlagvolumen (SV) abnahm und somit das Herzzeitvolumen nahezu konstant blieb. Der Anstieg der Herzfrequenz und die Abnahme des Schlagvolumen waren in der ARDS + AICH-Gruppe ausgeprägter als in der ARDS-Gruppe $(p<0,05)$. Der intrathorakale Blutvolumenindex (ITBI = ITBV bezogen auf die Körperoberfläche in $\mathrm{m}^{2}$ ) änderte sich nicht signifikant über den Versuchszeitraum und war auch zwischen den vier Gruppen konstant. 
Tabelle 7: Hämodynamik, gemessene und errechnete Parameter

\begin{tabular}{|c|c|c|c|c|}
\hline & $T_{0}$ & $T_{60}$ & $T_{120}$ & $T_{240}$ \\
\hline Control & $79 \pm 23$ & $86 \pm 16$ & $96 \pm 19$ & $85 \pm 20$ \\
\hline $\mathrm{AlCH}$ & $78 \pm 19$ & $102 \pm 23$ & $118 \pm 33^{*}$ & $112 \pm 34$ \\
\hline ARDS & $80 \pm 16$ & $110 \pm 21^{*}$ & $123 \pm 26^{*}$ & $120 \pm 19^{*}$ \\
\hline ARDS + AICH & $91 \pm 18$ & $148 \pm 30^{* \circ \wedge}$ & $157 \pm 27^{* \circ \wedge}$ & $145 \pm 37^{* 0}$ \\
\hline \multicolumn{5}{|l|}{ MAP, mmHg } \\
\hline Control & $76 \pm 15$ & $81 \pm 21$ & $80 \pm 14$ & $74 \pm 12$ \\
\hline $\mathrm{AlCH}$ & $77 \pm 19$ & $75 \pm 10$ & $67 \pm 7$ & $80 \pm 19$ \\
\hline ARDS & $78 \pm 13$ & $73 \pm 13$ & $77 \pm 14$ & $74 \pm 9$ \\
\hline $\mathrm{ARDS}+\mathrm{AICH}$ & $74 \pm 12$ & $71 \pm 9$ & $69 \pm 12$ & $67 \pm 9$ \\
\hline \multicolumn{5}{|l|}{ ZVD, mmHg } \\
\hline Control & $6,7 \pm 4$ & $6,7 \pm 4,5$ & $6,2 \pm 4,4$ & $7,2 \pm 3,7$ \\
\hline $\mathrm{AICH}$ & $6,6 \pm 2,5$ & $6,6 \pm 2,6$ & $5,9 \pm 3,2$ & $6,5 \pm 3,5$ \\
\hline ARDS & $6,0 \pm 1,6$ & $7,1 \pm 3,0$ & $8,3 \pm 4,3$ & $7,7 \pm 4,5$ \\
\hline $\mathrm{ARDS}+\mathrm{AICH}$ & $6,1 \pm 2,3$ & $7,6 \pm 2,2$ & $8,1 \pm 2,4$ & $8,8 \pm 3,1$ \\
\hline \multicolumn{5}{|l|}{$\mathrm{HZV}, \mathrm{I} / \mathrm{min}$} \\
\hline Control & $7,5 \pm 1,5$ & $7,1 \pm 1,6$ & $7,3 \pm 1,3$ & $6,9 \pm 1,0$ \\
\hline $\mathrm{AICH}$ & $8,0 \pm 1,3$ & $9,5 \pm 1,9$ & $13 \pm 5,4^{\circ}$ & $12,7 \pm 5,3^{\circ}$ \\
\hline ARDS & $7,6 \pm 1,6$ & $7,1 \pm 2,4$ & $7,8 \pm 2,8$ & $7,4 \pm 2,6$ \\
\hline $\mathrm{ARDS}+\mathrm{AICH}$ & $6,7 \pm 1,0$ & $7,5 \pm 2,7$ & $7,1 \pm 2,3$ & $8,6 \pm 2,6$ \\
\hline \multicolumn{5}{|l|}{$\mathrm{SV}, \mathrm{ml}$} \\
\hline Control & $82 \pm 18$ & $80 \pm 22$ & $78 \pm 17$ & $81 \pm 20$ \\
\hline$\overline{\mathrm{AlCH}}$ & $92 \pm 25$ & $89 \pm 25$ & $93 \pm 27$ & $99 \pm 25$ \\
\hline ARDS & $90 \pm 15$ & $59 \pm 14^{*}$ & $67 \pm 17^{*}$ & $59 \pm 13^{*}$ \\
\hline $\mathrm{ARDS}+\mathrm{AICH}$ & $86 \pm 17$ & $56 \pm 19^{\star \circ}$ & $46 \pm 14^{* \circ \wedge}$ & $59 \pm 17^{*}$ \\
\hline \multicolumn{5}{|l|}{ MPAP, $\mathrm{mmHg}$} \\
\hline Control & $23 \pm 5$ & $21 \pm 2$ & $21 \pm 2$ & $21 \pm 4$ \\
\hline $\mathrm{AICH}$ & $20 \pm 2$ & $22 \pm 4$ & $23 \pm 3$ & $21 \pm 4$ \\
\hline ARDS & $21 \pm 4$ & $39 \pm 4^{\star \circ}$ & $40 \pm 6^{\star \circ}$ & $40 \pm 6^{\star \circ}$ \\
\hline $\mathrm{ARDS}+\mathrm{AICH}$ & $23 \pm 4$ & $43 \pm 2^{\star \circ \wedge}$ & $43 \pm 2^{\star \circ}$ & $43 \pm 8^{\star \circ}$ \\
\hline \multicolumn{5}{|l|}{ ITBI, $\mathrm{ml} / \mathrm{m}^{2}$} \\
\hline Control & $729 \pm 125$ & $704 \pm 91$ & $712 \pm 83$ & $755 \pm 98$ \\
\hline $\mathrm{AlCH}$ & $824 \pm 140$ & $847 \pm 130$ & $830 \pm 123$ & $892 \pm 152$ \\
\hline ARDS & $796 \pm 135$ & $765 \pm 91$ & $809 \pm 100$ & $758 \pm 128$ \\
\hline $\mathrm{ARDS}+\mathrm{AICH}$ & $702 \pm 56$ & $788 \pm 164$ & $663 \pm 107$ & $738 \pm 130$ \\
\hline
\end{tabular}

Darstellung der hämodynamischen Messwerte (HF = Herzfrequenz, MAP = mittlerer arterieller Druck, $Z V D=$ zentralvenöser Druck, HZV = Herzzeitvolumen, $S V=$ Schlagvolumen, MPAP = mittlerer pulmonalarterieller Druck, ITBI = intrathorakaler Blutvolumen-Index) als Mittelwerte mit Standardabweichung von je 7 Tieren pro Gruppe. Control: Kontrollgruppe; AICH: Acute Intracranial Hypertension; ARDS: Acute Respiratory Distress Syndrome; AICH + ARDS: Acute Intracranial Hypertension + Acute Respiratory Distress Syndrome. ${ }^{*} p<0,05$ vs. $T_{0} ;{ }^{\circ} p<0.05$ vs. Control: ${ }^{\wedge} p<0.05$ vs. ARDS. (Publiziert in Intensive Care Med (2011) 37:1182-1191, Electronic supplementary material (ESM), Table. 1) 
Abbildung 26: Entwicklung der Herzfrequenz in allen Gruppen

Herzfrequenz (HF) aller Gruppen - $T_{0}-T_{240}$

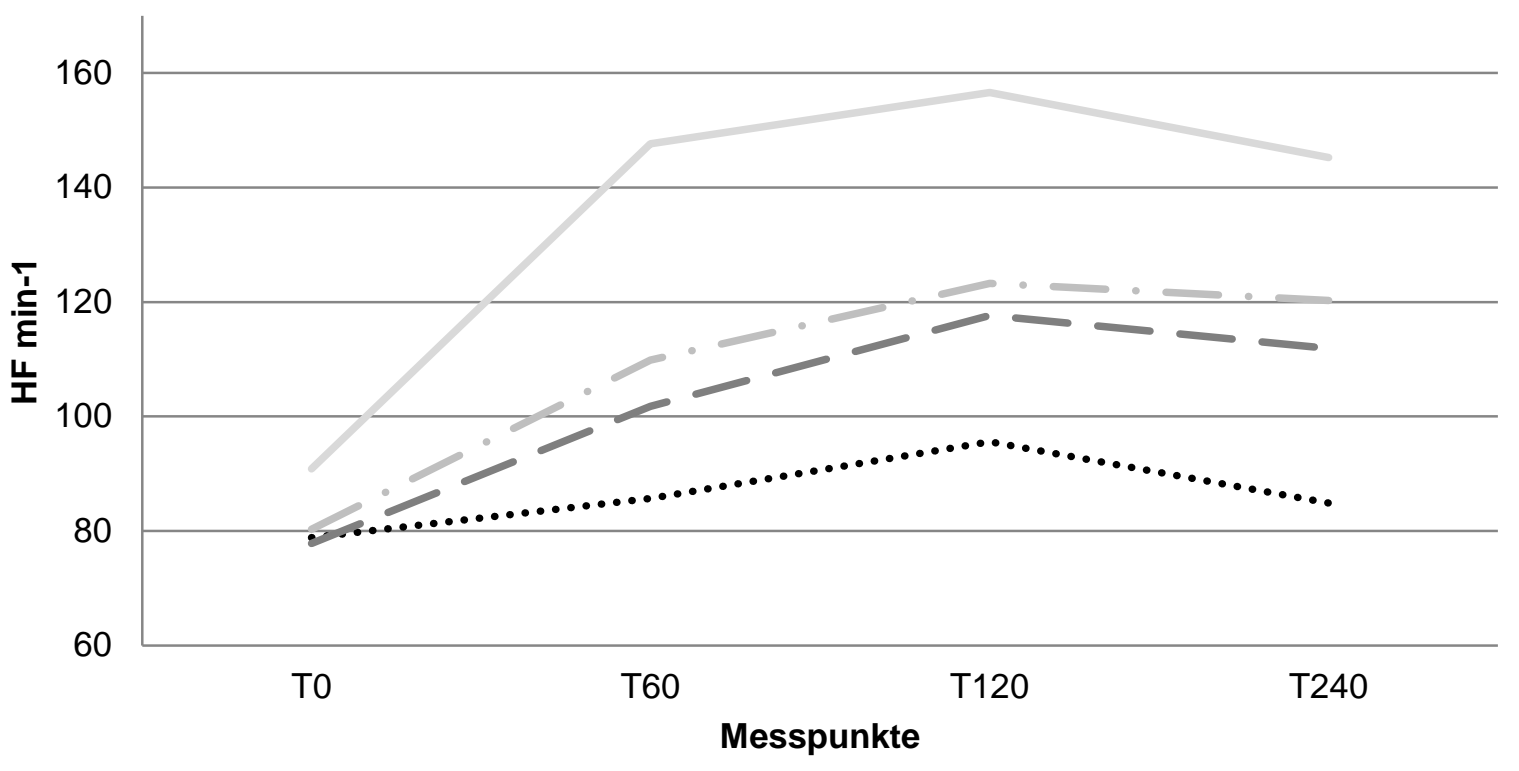

..... Control - -AICH - ARDS $-\mathrm{ARDS}+\mathrm{AICH}$

Entwicklung der Herzfrequenz in allen Gruppen über 240 Minuten. Die deutlichsten Veränderungen der HF fanden sich in den Gruppen ARDS und ARDS + AICH. Signifikante Unterschiede zwischen den Gruppen Control und AICH gab es nicht. Zwischen den Gruppen ARDS und ARDS + AICH war die HF zu den Messpunkten $T_{60}$ und $T_{120}$ signifikant $(p<0,05)$ unterschiedlich. 
Abbildung 27: Entwicklung des Schlagvolumens in allen Gruppen

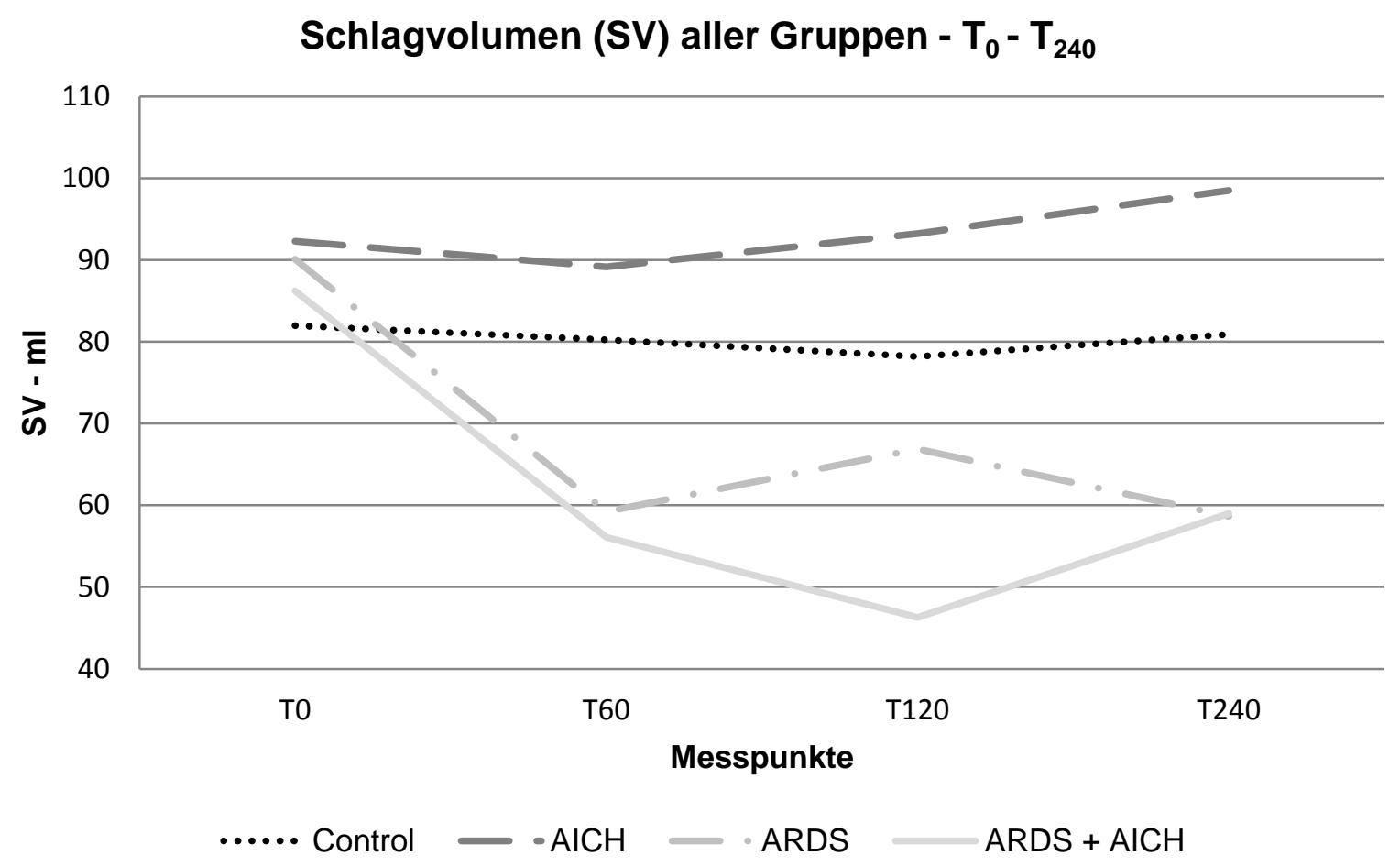

Entwicklung des Schlagvolumens in allen Gruppen über 240 Minuten. Die deutlichsten Veränderungen des SV fanden sich in den Gruppen ARDS und ARDS + $A I C H$. Signifikante Unterschiede zwischen den Gruppen Control und AICH gab es nicht. Zwischen den Gruppen ARDS und ARDS + AICH war das SV zum Messpunkt $T_{120}$ signifikant $(p<0,05)$ unterschiedlich. 
Abbildung 28: Entwicklung des Herzzeitvolumens in allen Gruppen

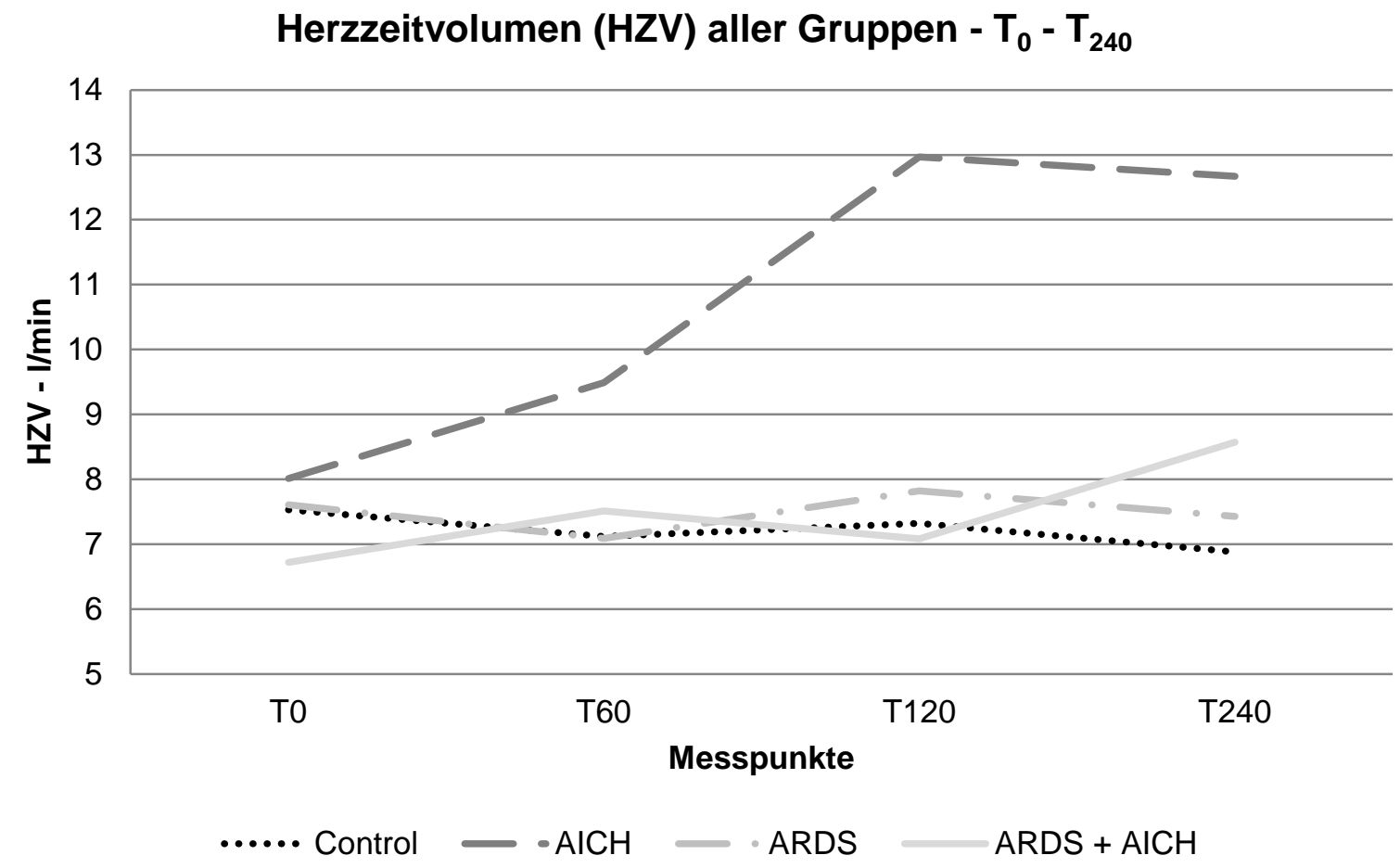

Entwicklung des Herzzeitvolumens in allen Gruppen über 240 Minuten. Das Herzzeitvolumen der drei Gruppen Control, ARDS und ARDS + AICH blieb über die gesamte Versuchsdauer annähernd konstant und zeigte keine signifikanten Unterschiede. In der AICH-Gruppe stieg das HZV verglichen mit der Kontrollgruppe zu den Messpunkten $T_{120}$ und $T_{240}$ signifikant an $(p<0,05)$. 


\subsection{Gasaustausch}

Die Entwicklung des Gasaustauschs über alle Gruppen vom Zeitpunkt $T_{0}$ bis $T_{240}$ kann der Tabelle 8 entnommen werden.

Der arterielle Sauerstoffpartialdruck $\left(\mathrm{PaO}_{2}\right)$ sowie der arterielle Kohlendioxidpartialdruck $\left(\mathrm{PaCO}_{2}\right)$ waren sowohl in der Kontrollgruppe als auch in der AICH-Gruppe über das gesamte Experiment konstant. In den Gruppen ARDS und AICH + ARDS nahm der arterielle Sauerstoffpartialdruck jeweils nach Induktion der Lungenschädigung ab (niedrigster Wert: $65 \mathrm{mmHg}$ ), während der arterielle Kohlendioxidpartialdruck in diesen beiden Gruppen bis auf ein Maximum von $65 \mathrm{mmHg}$ anstieg. Der niedrigste arterielle Sauerstoffpartialdruck fand sich in der AICH + ARDS-Gruppe. 
Tabelle 8: Gasaustausch, gemessene Parameter

\begin{tabular}{|c|c|c|c|c|}
\hline & $\mathrm{T}_{0}$ & $T_{60}$ & $T_{120}$ & $T_{240}$ \\
\hline \multicolumn{5}{|l|}{$\mathrm{PaO}_{2}, \mathrm{mmHg}$} \\
\hline Control & $486 \pm 55$ & $456 \pm 78$ & $468 \pm 96$ & $470 \pm 74$ \\
\hline $\mathrm{AICH}$ & $451 \pm 53$ & $471 \pm 63$ & $395 \pm 46^{*}$ & $431 \pm 109$ \\
\hline ARDS & $499 \pm 60$ & $151 \pm 91^{* \circ}$ & $171 \pm 23^{* \circ}$ & $150 \pm 98^{* \circ}$ \\
\hline $\mathrm{ARDS}+\mathrm{AICH}$ & $493 \pm 66$ & $155 \pm 54^{\star \circ}$ & $140 \pm 37^{\star \circ}$ & $110 \pm 38^{* 0}$ \\
\hline \multicolumn{5}{|l|}{$\mathrm{PaCO}_{2}, \mathrm{mmHg}$} \\
\hline Control & $42 \pm 5$ & $44 \pm 8$ & $42 \pm 5$ & $35 \pm 5$ \\
\hline $\mathrm{AlCH}$ & $41 \pm 2$ & $46 \pm 9$ & $44 \pm 9$ & $36 \pm 5$ \\
\hline ARDS & $39 \pm 7$ & $65 \pm 16^{\star \circ}$ & $65 \pm 13^{* \circ}$ & $63 \pm 6^{\star \circ}$ \\
\hline $\mathrm{ARDS}+\mathrm{AICH}$ & $39 \pm 2$ & $63 \pm 9^{* \circ}$ & $61 \pm 7^{\star \circ}$ & $59 \pm 9 * \circ$ \\
\hline \multicolumn{5}{|l|}{$\mathrm{pHa}$} \\
\hline Control & $7,42 \pm 0,08$ & $7,36 \pm 0,07$ & $7,38 \pm 0,08$ & $7,40 \pm 0,06$ \\
\hline $\mathrm{AlCH}$ & $7,40 \pm 0,06$ & $7,31 \pm 0,10$ & $7,32 \pm 0,11$ & $7,46 \pm 0,11$ \\
\hline ARDS & $7,41 \pm 0,12$ & $7,17 \pm 0,09^{\circ}$ & $7,19 \pm 0,07^{\circ}$ & $7,23 \pm 0,05^{\circ}$ \\
\hline $\mathrm{ARDS}+\mathrm{AICH}$ & $7,37 \pm 0,08$ & $7,19 \pm 0,06^{\circ}$ & $7,21 \pm 0,08^{\circ}$ & $7,26 \pm 0,07^{\circ}$ \\
\hline \multicolumn{5}{|l|}{$\mathrm{SaO}_{2}, \%$} \\
\hline Control & $99,9 \pm 0,2$ & $99,9 \pm 0,1$ & $99,9 \pm 0,2$ & $99,9 \pm 0,1$ \\
\hline $\mathrm{AICH}$ & $99,9 \pm 0,1$ & $99,9 \pm 0,3$ & $99,8 \pm 0,3$ & $99,9 \pm 0,2$ \\
\hline ARDS & $99,8 \pm 0,2$ & $95,7 \pm 4,4^{*}$ & $95,5 \pm 4,0^{*}$ & $94,8 \pm 5,8$ \\
\hline $\mathrm{ARDS}+\mathrm{AICH}$ & $99,9 \pm 0,2$ & $98,1 \pm 1,5^{\star}$ & $97,7 \pm 1,5^{\star}$ & $93,7 \pm 6,5^{\star}$ \\
\hline \multicolumn{5}{|l|}{$\mathrm{SvO}_{2}, \%$} \\
\hline Control & $86,0 \pm 6,8$ & $90,9 \pm 2,9$ & $88,6 \pm 4,6$ & $84,3 \pm 9,6$ \\
\hline$\overline{\mathrm{AlCH}}$ & $92,2 \pm 4,3$ & $94,8 \pm 3,9$ & $90,9 \pm 9,3$ & $89,6 \pm 7,2$ \\
\hline ARDS & $85,0 \pm 7,3$ & $62,5 \pm 9,5^{\star \circ}$ & $65,2 \pm 9,8^{* \circ}$ & $63,4 \pm 5,4^{\star \circ}$ \\
\hline $\mathrm{ARDS}+\mathrm{AICH}$ & $89,3 \pm 3,0$ & $72,5 \pm 6,7^{\star \circ}$ & $76,0 \pm 8,8^{*}$ & $66,7 \pm 9,4^{* \circ}$ \\
\hline \multicolumn{5}{|l|}{ Shunt, \% } \\
\hline Control & $7,2 \pm 2,8$ & $8,7 \pm 3,8$ & $8,2 \pm 3,7$ & $8,2 \pm 3,6$ \\
\hline $\mathrm{AICH}$ & $9,0 \pm 2,7$ & $7,8 \pm 2,8$ & $11,7 \pm 2,1^{*}$ & $9,9 \pm 5,3$ \\
\hline ARDS & $6,8 \pm 3,0$ & $22,9 \pm 4,3^{* \circ}$ & $21,9 \pm 5,9^{* 0}$ & $23,0 \pm 4,7^{* 0}$ \\
\hline $\mathrm{ARDS}+\mathrm{AICH}$ & $7,4 \pm 3,2$ & $22,6 \pm 2,3^{\star \circ}$ & $23,9 \pm 1,8^{* 0}$ & $26,4 \pm 3,6^{* \circ}$ \\
\hline
\end{tabular}

Darstellung der Gasaustauschparameter $\left(\mathrm{PaO}_{2}=\right.$ arterieller Sauerstoffpartialdruck, $\mathrm{PaCO}_{2}=$ arterieller Kohlendioxidpartialdruck, $\mathrm{pHa}=$ arterielle Wasserstoffionenkonzentration, $\mathrm{SaO}_{2}=$ pulsoxymetrische Sauerstoffsättigung, Shunt = Shuntvolumen) als Mittelwerte mit Standardabweichung von je 7 Tieren pro Gruppe. Control: Kontrollgruppe; AICH: Acute Intracranial Hypertension; ARDS: Acute Respiratory Distress Syndrome; AICH + ARDS: Acute Intracranial Hypertension + Acute Respiratory Distress Syndrome. ${ }^{*} p<0,05$ vs. $T_{0} ;{ }^{\circ} p<0.05$ vs. Control: ${ }^{\wedge} p<0,05$ vs. ARDS. (Publiziert in Intensive Care Med (2011) 37:1182-1191, Electronic supplementary material (ESM), Table. 2) 


\subsection{CT-Analyse der Lunge}

\subsubsection{Analyse der gesamten Lunge}

Die mittlere Lungendichte, ausgedrückt in Hounsfield Units (HU), der Gasgehalt und die prozentuale Verteilung von normal, wenig und nicht belüftetem Lungengewebe können der Tabelle 9 entnommen werden. Die Abbildungen 29 und 30 veranschaulichen die Ergebnisse der pulmonalen Dichtemessung in Hounsfield Units, Abbildung 31 und 32 die Entwicklung des Gasgehaltes und die Abbildungen 33 - 38 den Verlauf normal, wenig und nicht ventilierter Lungenareale in Prozent.

In der Kontrollgruppe nahm die Dichte des Lungengewebes vom Zeitpunkt $T_{240}$ zum Zeitpunkt $T_{0}$ im Mittel um $38 \mathrm{HU}( \pm 23)$ ab. Die Dichte nahm in der Gruppe AICH um 28 $\mathrm{HU}( \pm 25)$, in der Gruppe ARDS um $431 \mathrm{HU}( \pm 80)$ und in der Gruppe AICH + ARDS um $509 \mathrm{HU}( \pm 59)$ zu. Bezüglich der Hounsfield Units konnte ein signifikanter Unterschied $(p<0,05)$ zwischen der Kontrollgruppe und der AICH-Gruppe zu den Zeitpunkten $T_{60}$, $T_{120}$ und $T_{240}$ festgestellt werden. Zwischen den Gruppen ARDS und AICH + ARDS fanden sich signifikante $(p<0,05)$ Unterschiede der Hounsfield Units zu den Zeitpunkten $\mathrm{T}_{120}$ und $\mathrm{T}_{240}$. Es kam also im Vergleich der Gruppen Control und AICH sowohl zu einer Veränderung des Herzzeitvolumens an den Messpunkten $T_{120}$ sowie $T_{240}$ als auch zu einer Veränderung der Lungenparenchymdichte. Zwischen den Gruppen ARDS und ARDS + AICH konnte ebenfalls eine Veränderung der Lungenparenchymdichte beobachtet werden, allerdings ohne eine signifikante Veränderung des Herzzeitvolumens.

Diese Dichtezunahmen gingen stets mit einer Abnahme des Gasgehaltes (ml Gas/ Gramm Gewebe) einher. Die Abnahme des Gasgehaltes war zwischen den Gruppen Control und AICH zu den Zeitpunkten $T_{60}, T_{120}$ und $T_{240}$ signifikant $(p<0,05)$ und zwischen der ARDS- und ARDS + AICH-Gruppe zu allen Messpunkten.

Des Weiteren konnte eine signifikante Abnahme der normal ventilierten Lungenareale in der Gruppe AICH im Vergleich zur Kontrollgruppe zu den Zeitpunkten $T_{0}, T_{60}, T_{120}$ und $T_{240}$ und eine Zunahme der schlecht und nicht ventilierten Bereiche zu den Zeitpunkten $T_{0}, T_{60}, T_{120}$ und $T_{240}$ festgestellt werden. In der Gruppe ARDS + AICH nahm der Anteil normal ventilierter Lungenareale zu den Zeitpunkten $T_{60}$ und $T_{240}$ im Vergleich zur ARDS-Gruppe signifikant $(p<0,05)$ ab und der Anteil nicht ventilierter Bereiche im Vergleich zur ARDS-Gruppe zum Zeitpunkt $\mathrm{T}_{120} \mathrm{zu}$. 
Tabelle 9: CT-Daten der gesamten Lunge $T_{240}-T_{0}$ (Seg. 1 - 10)

\section{Hounsfield Units}

Control

$\mathrm{AICH}$

ARDS

ARDS + AICH

Gasgehalt, $\mathbf{m l} / \mathbf{g}$

Control

$\mathrm{AICH}$

ARDS

$\mathrm{ARDS}+\mathrm{AICH}$

normal belüftetes

Control

$\mathrm{AlCH}$

ARDS

ARDS+AICH

schlecht belüftetes

Control

$\mathrm{AICH}$

ARDS

$\mathrm{ARDS}+\mathrm{AICH}$

nicht belüftetes

Control

$\mathrm{AICH}$

ARDS

$\mathrm{ARDS}+\mathrm{AICH}$
$\mathbf{T}_{0}$

$-673 \pm 20$

$-643 \pm 20^{\circ}$

$-636 \pm 30^{\circ}$

$-666 \pm 33^{\wedge}$

$2,36 \pm 0,19$

$2,02 \pm 0,16$

$1,85 \pm 0,25^{\circ}$

$2,18 \pm 0,32^{\circ \wedge}$

$83,5 \pm 4,0$

$81,5 \pm 3,9^{\circ}$

$81,7 \pm 6,6$

$83,7 \pm 5,7^{\wedge}$

$11,3 \pm 3,9$
$13,1 \pm 3,8^{\circ}$
$15,8 \pm 7,2^{\circ}$
$13,6 \pm 6,4^{\wedge}$

$2,2 \pm 2,1$

$3,4 \pm 1,1^{\circ}$

$1,8 \pm 2,0$

$1,5 \pm 1,7$
$T_{60}$

$-679 \pm 25$

$-603 \pm 20^{* \circ}$

$-178 \pm 103^{* 0}$

$-154 \pm 113^{* 0}$

$2,38 \pm 0,26$

$1,77 \pm 0,14^{* \circ}$

$0,55 \pm 0,13^{* \circ}$

$0,41 \pm 0,13^{* 0 \wedge}$

$83,6 \pm 5,1$

$76,5 \pm 4,3^{* 0}$

$9,4 \pm 6,9^{* \circ}$

$7,4 \pm 7,6^{* 0 \wedge}$

$11,2 \pm 5,1$

$17,9 \pm 4,5^{\star \circ}$

$42,5 \pm 19,1^{* \circ}$

$39,1 \pm 20,7^{\star \circ}$

$2,1 \pm 2,4$

$3,8 \pm 1,2^{* 0}$

$47,8 \pm 25,1^{* \circ}$

$53,1 \pm 27,1^{\text {*。 }}$
$T_{120}$

$\mathbf{T}_{240}$

$-682 \pm 25^{*}$

$-711 \pm 25^{*}$

$-624+23^{* 0}$

$-615 \pm 27^{* 0}$

$-241 \pm 93^{* \circ}$

$-205 \pm 99 * 0$

$-166 \pm 124^{\star \circ \wedge}$

$-158 \pm 122^{* \text { ०ᄉ }}$

$2,27 \pm 0,23$

$2,65 \pm 0,28^{*}$

$1,92 \pm 0,15^{\circ}$

$1,83 \pm 0,20$ *o

$0,72 \pm 0,14^{* \circ}$

$0,66 \pm 0,16^{* \circ}$

$0,43 \pm 0,15^{\star \circ \wedge}$

$0,45 \pm 0,15^{* \text { ○ }}$

$83,7 \pm 4,8$

$78,5 \pm 4,5^{\star \circ}$

$84,6 \pm 34,0$

$15,4 \pm 8,6^{\star 0}$

$78,3 \pm 6,0^{* \circ}$

$8,0 \pm 8,9 * 0$

$13,1 \pm 9,2^{* \circ}$

$8,8 \pm 9,4^{* \circ \wedge}$

$10,6 \pm 4,5$

$16,4 \pm 4,8^{* \circ}$

$8,9 \pm 4,0$ *

$48,0 \pm 10,9^{* \circ}$

$16,9 \pm 6,5^{\star \circ}$

$42,2 \pm 12,4^{\text {*o }}$

$40,3 \pm 22,7^{\star \circ}$

$36,1 \pm 21,3^{* \circ}$

$2,2 \pm 2,3$

$1,9 \pm 2,1^{*}$

$3,2 \pm 1,1^{\circ}$

$3,0 \pm 1,0^{\circ}$

$36,0 \pm 18,7^{* \circ}$

$44,3 \pm 20,9^{* \circ}$

$51,1 \pm 29,7^{\star \circ \wedge}$

$54,4 \pm 29,1^{* 0}$

Darstellung als Mittelwerte mit Standardabweichung von je 7 Tieren pro Gruppe. Control: Kontrollgruppe; AICH: Acute Intracranial Hypertension; ARDS: Acute Respiratory Distress Syndrome; AICH + ARDS: Acute Intracranial Hypertension + Acute Respiratory Distress Syndrome. * $p<0,05$ vs. $T_{0} ;{ }^{\circ} p<0,05$ vs. Control: $\wedge$ $p<0,05$ vs. ARDS. (Publiziert in Intensive Care Med (2011) 37:1182-1191, Electronic supplementary material (ESM), Table. 3) 
Abbildung 29: HU der gesamten Lunge - Control vs. AlCH

\section{HU der gesamten Lunge}

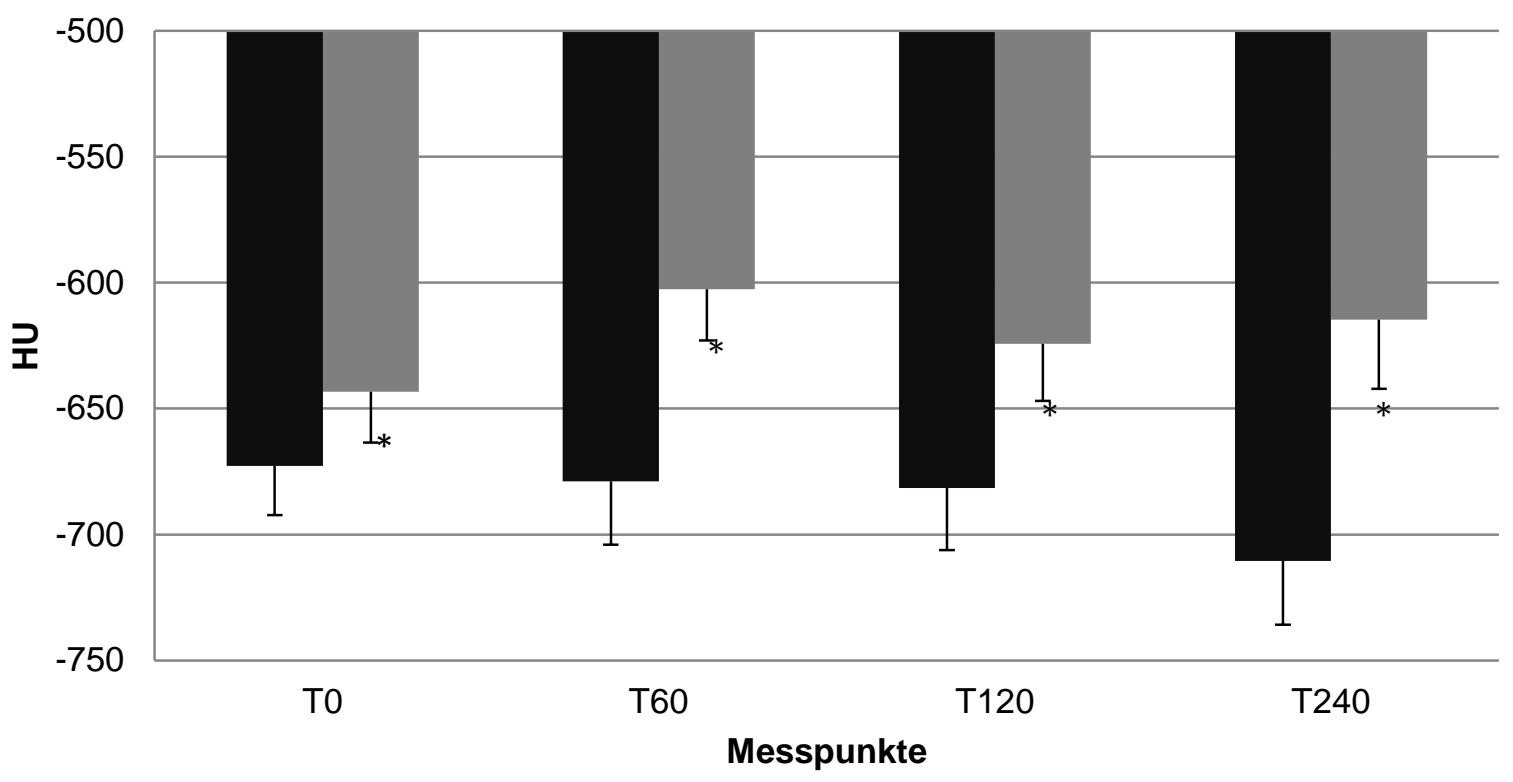

- HU - Control $\quad \mathrm{HU}-\mathrm{AICH}$

Entwicklung der gesamten Lungenparenchymdichte für die Gruppen Control und AlCH über 240 Minuten. Es ist zu erkennen, dass zum Zeitpunkt $T_{0}$ die Parenchymdichte der Kontrollgruppe geringer ist als die Dichte der AlCH-Gruppe. In der Kontrollgruppe verringert sich die Dichte weiter, während sie in der $\mathrm{AlCH}$ Gruppe zunimmt (weniger negativ). Signifikante Unterschiede $(p<0,05)$ fanden sich an den Messpunkten $T_{0}, T_{60}, T_{120}$ und $T_{240} .\left({ }^{*}=p<0,05\right)$ 
Abbildung 30: HU der gesamten Lunge - ARDS vs. ARDS + AICH

HU - ARDS vs. ARDS + AICH

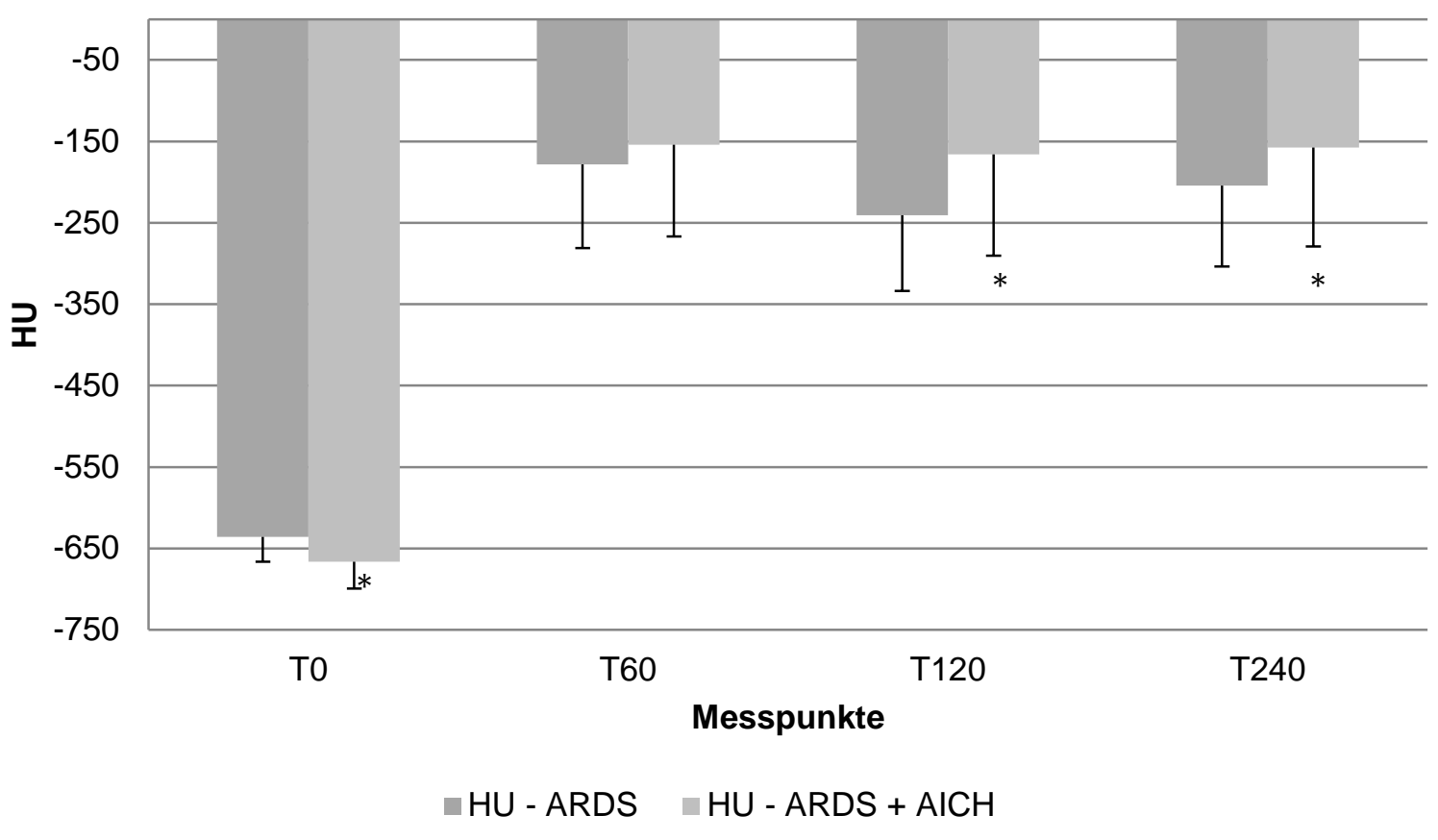

Entwicklung der gesamten Lungenparenchymdichte für die Gruppen ARDS und ARDS + AICH über 240 Minuten. Es ist zu erkennen, dass die Parenchymdichte der ARDS + AICH-Gruppe im Vergleich zur ARDS-Gruppe weiter zunimmt (weniger negativ). Signifikante Unterschiede gab es an den Messpunkten $T_{0}, T_{120}$ und $T_{240 .}\left(^{*}=\right.$ $p<0,05)$ 
Abbildung 31: Gasgehalt der gesamten Lunge - $\mathrm{ml} / \mathrm{g}$ - Control vs. AICH

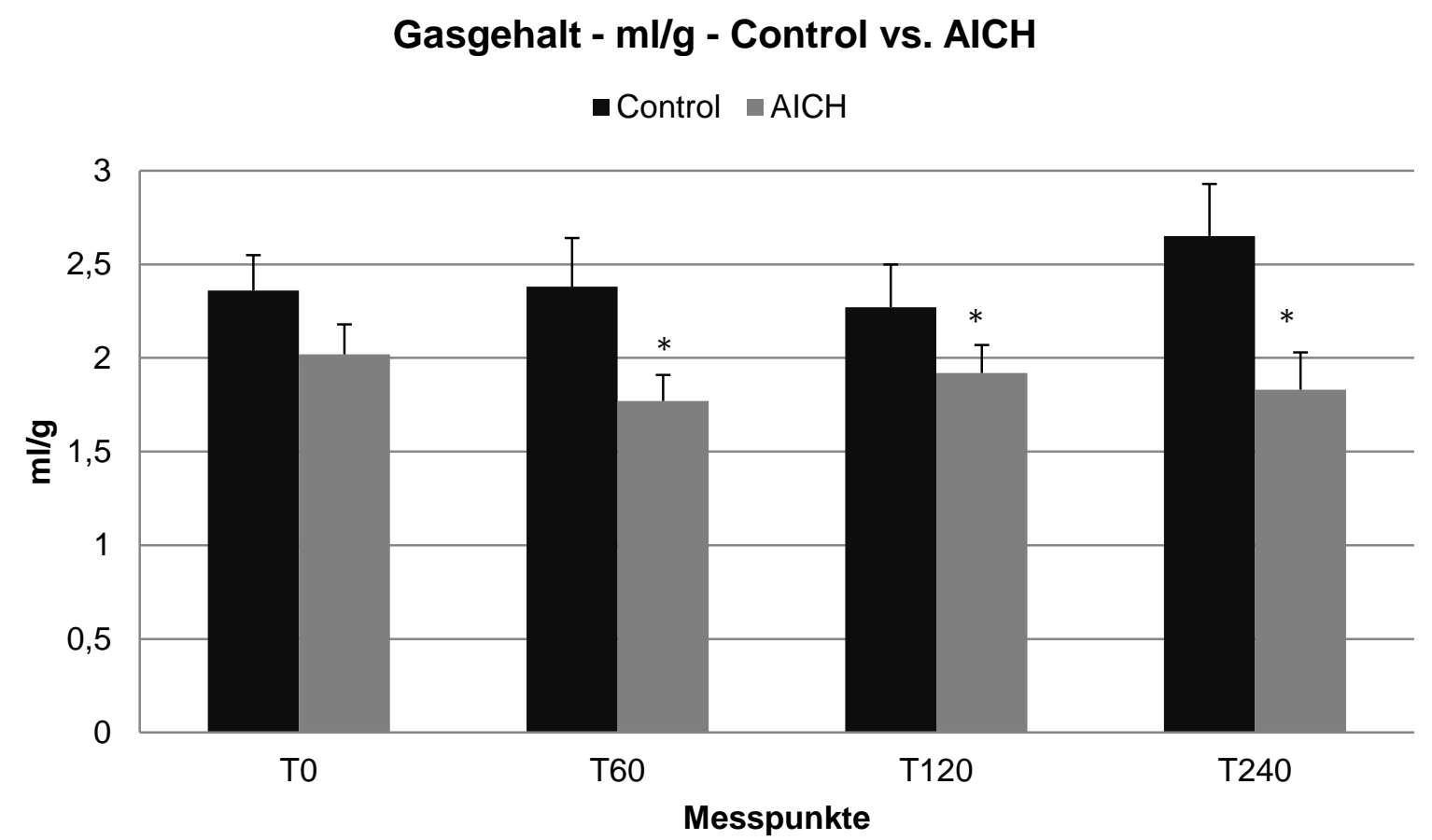

Entwicklung des Gasgehaltes für die Gruppen Control und AlCH über 240 Minuten. Zwar war der Gasgehalt in der Kontrollgruppe bereits zum Zeitpunkt $T_{0}$ besser, allerdings nimmt er im Verlauf weiter zu, während der Gasgehalt in der AlCH-Gruppe weiter abnimmt. Der unterschiedliche Gasgehalt war zum Zeitpunkt To nicht signifikant. An den Messpunkten $T_{60}, T_{120}$, und $T_{240}$ fand sich ein signifikanter Unterschied $(p<0,05)$ im Vergleich zur Kontrollgruppe. $\left({ }^{*}=p<0,05\right)$ 
Abbildung 32: Gasgehalt der gesamten Lunge - ml/g - ARDS vs. ARDS + AICH

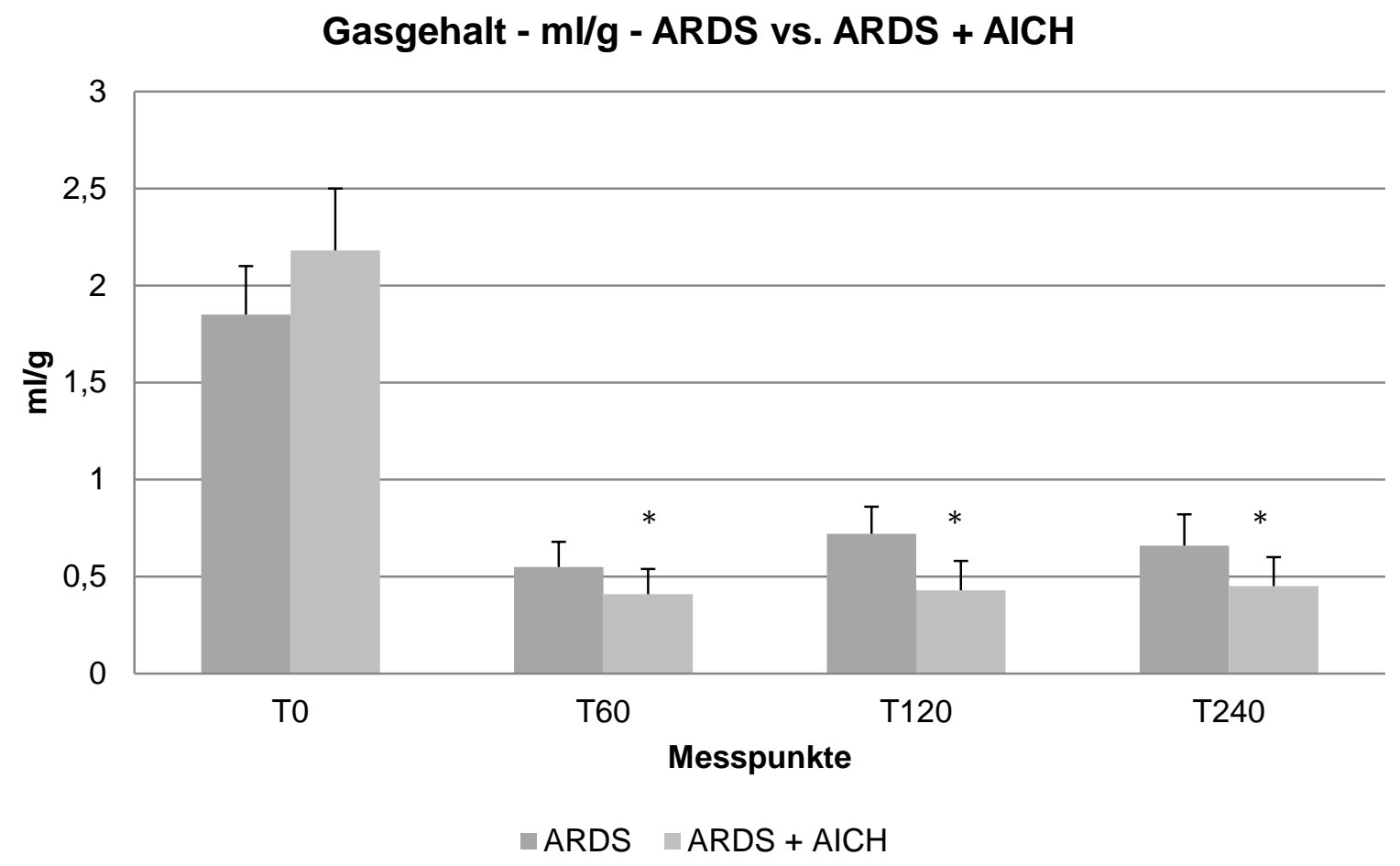

Entwicklung des Gasgehaltes in den Gruppen ARDS und ARDS + AlCH über 240 Minuten. Der Gasgehalt der ARDS-Gruppe war zum Zeitpunkt $T_{0}$ signifikant geringer als in der ARDS + AICH-Gruppe. Im weiteren Verlauf kehrt sich dies um. Die ARDS-Gruppe hat zu den Zeitpunkten $T_{60}, T_{120}$ und $T_{240}$ einen signifikant höheren Gasgehalt als die ARDS + AlCH-Gruppe. Diese Ergebnisse passen auch zu der gemessenen Lungenparenchymdichte (siehe Abbildung 29). ${ }^{*}=p<0,05$ ) 
Abbildung 33: Normal ventilierte Lunge - Control vs. AICH

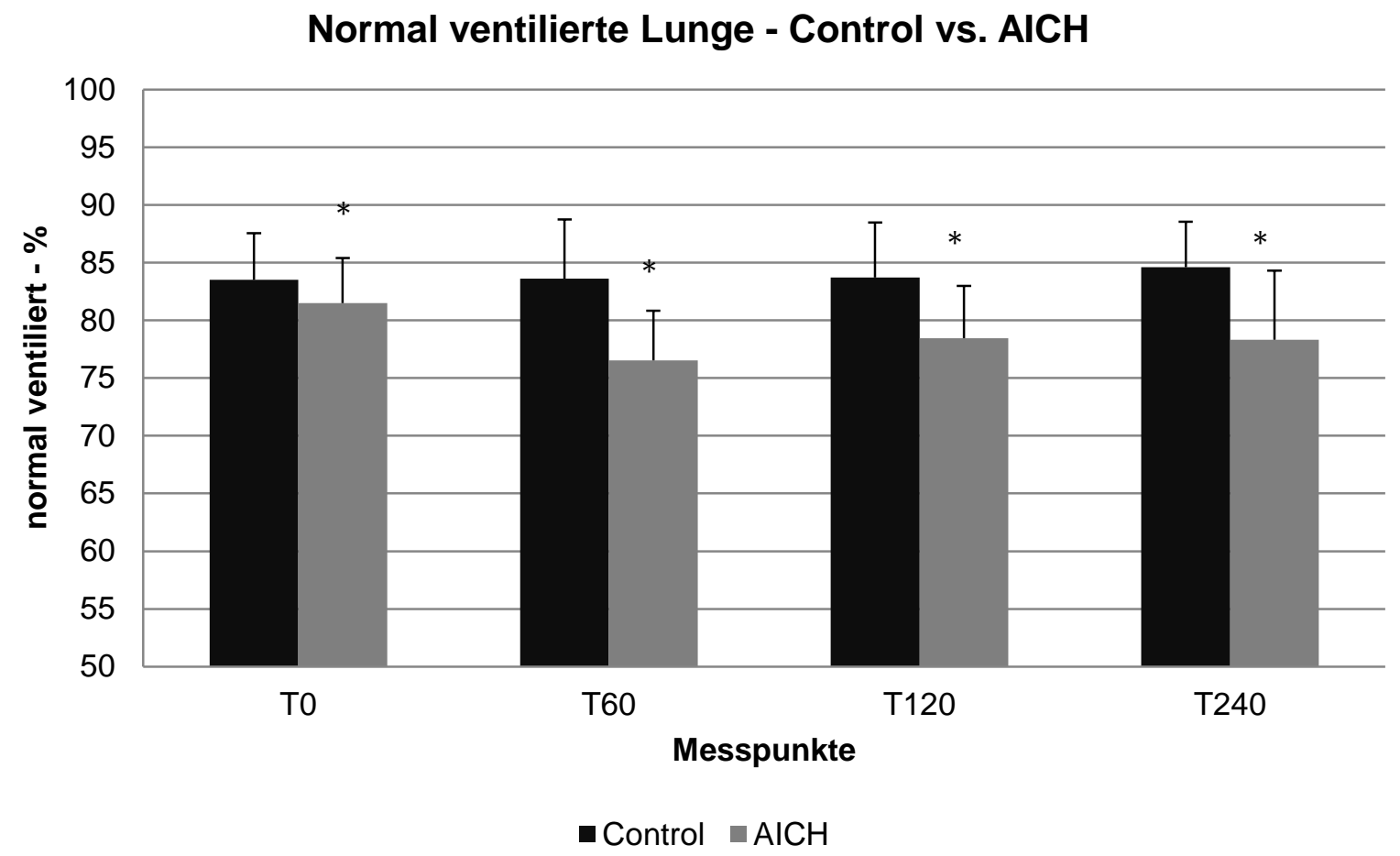

Entwicklung normal ventilierter Lungenbereiche für die Gruppen Control und $\mathrm{AlCH}$ über 240 Minuten. Signifikante Unterschiede bezüglich der normal ventilierten Lungenabschnitte fanden sich zu allen Messpunkten. Wichtig ist, dass es zu den Messpunkten $T_{60}, T_{120}$ und $T_{240} z u$ einer weiteren Verminderung normal ventilierter Lungenareale in der Gruppe AlCH kommt, während sich der Anteil normal ventilierter Bereiche in der Kontrollgruppe leicht erhöht. $\left({ }^{*}=p<0,05\right)$ 
Abbildung 34: Normal ventilierte Lunge - ARDS vs. ARDS + AICH

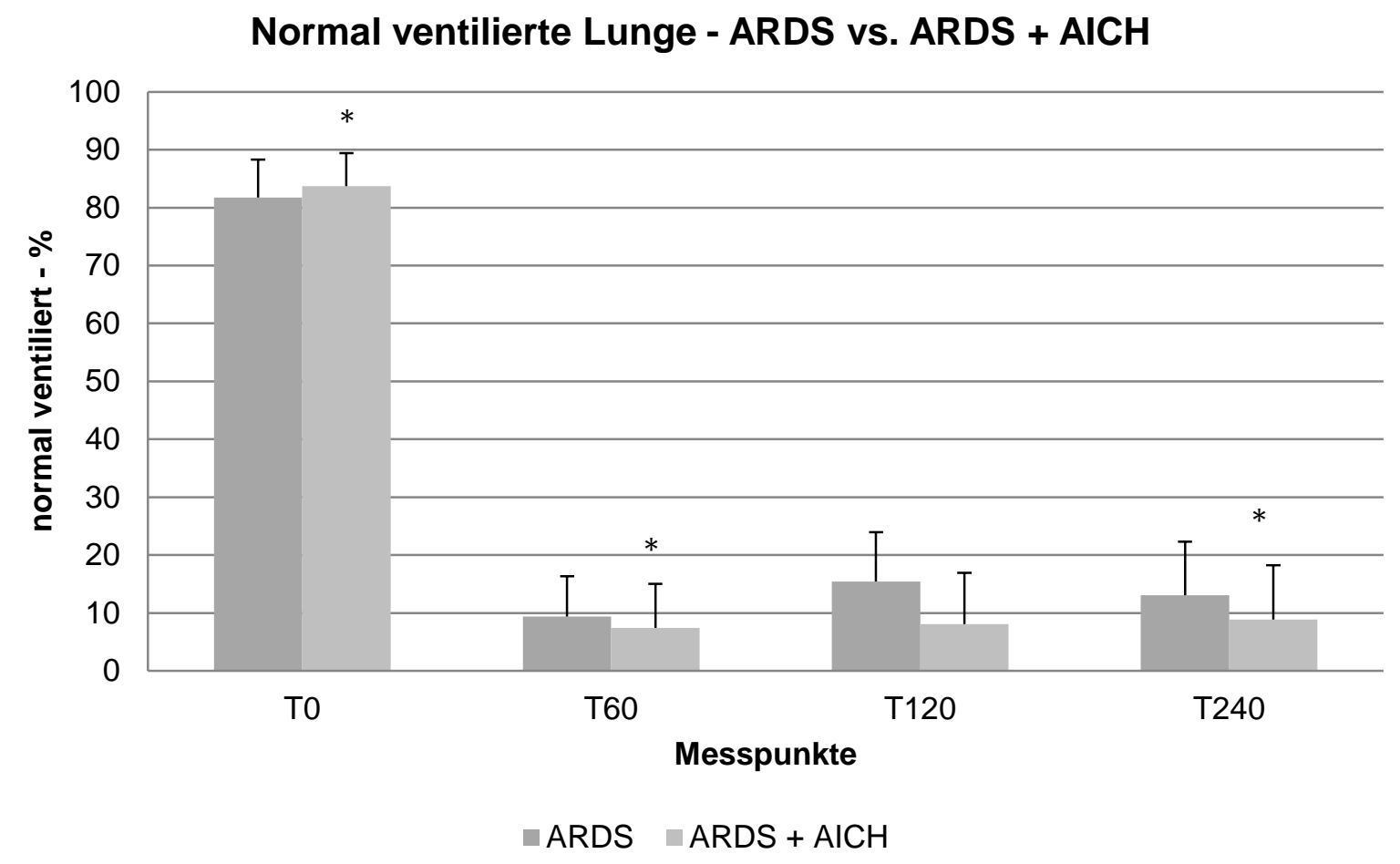

Entwicklung normal ventilierter Lungenbereiche für die Gruppen ARDS und ARDS + AICH über 240 Minuten. Im direkten Vergleich der Gruppen ARDS und ARDS + AlCH finden sich signifikante Unterschiede zu den Messpunkten $T_{0}, T_{60}$ und $T_{240}$. Der Ausgangswert für normal ventilierte Lungenbereiche war in der ARDS + AICHGruppe signifikant höher. Interessanterweise kommt es trotz dieses anfänglichen Unterschiedes zu einer signifikant größeren Abnahme normal ventilierter Areale in $\operatorname{der}$ ARDS + AICH-Gruppe. $\left({ }^{*}=p<0,05\right)$ 
Abbildung 35: Schlecht ventilierte Lunge - CG vs. AICH

Schlecht ventilierte Lunge - Control vs. AICH

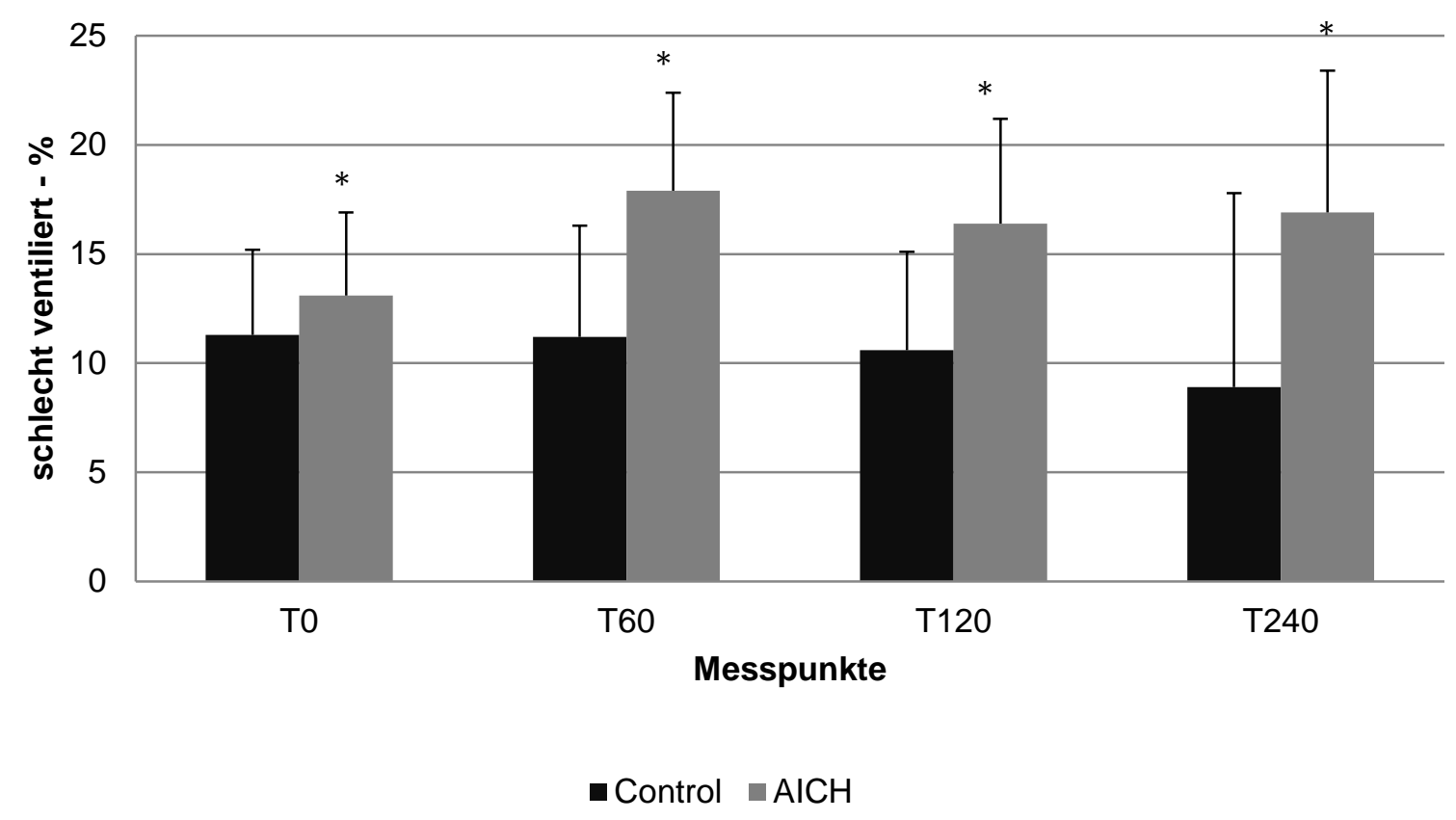

Entwicklung schlecht ventilierter Lungenbereiche für die Gruppen Control und $\mathrm{AlCH}$ über 240 Minuten. Signifikante Unterschiede zeigten sich zu allen Messpunkten. Im Verlauf kam es zu einer weiteren Zunahme schlecht ventilierter Bereiche in der AlCH-Gruppe, während sich deren Anteil in der Kontrollgruppe über 240 Minuten verringerte. $\left({ }^{*}=p<0,05\right)$ 
Abbildung 36: Schlecht ventilierte Lunge - ARDS vs. ARDS + AICH

Schlecht ventilierte Lunge - ARDS vs. ARDS + AICH

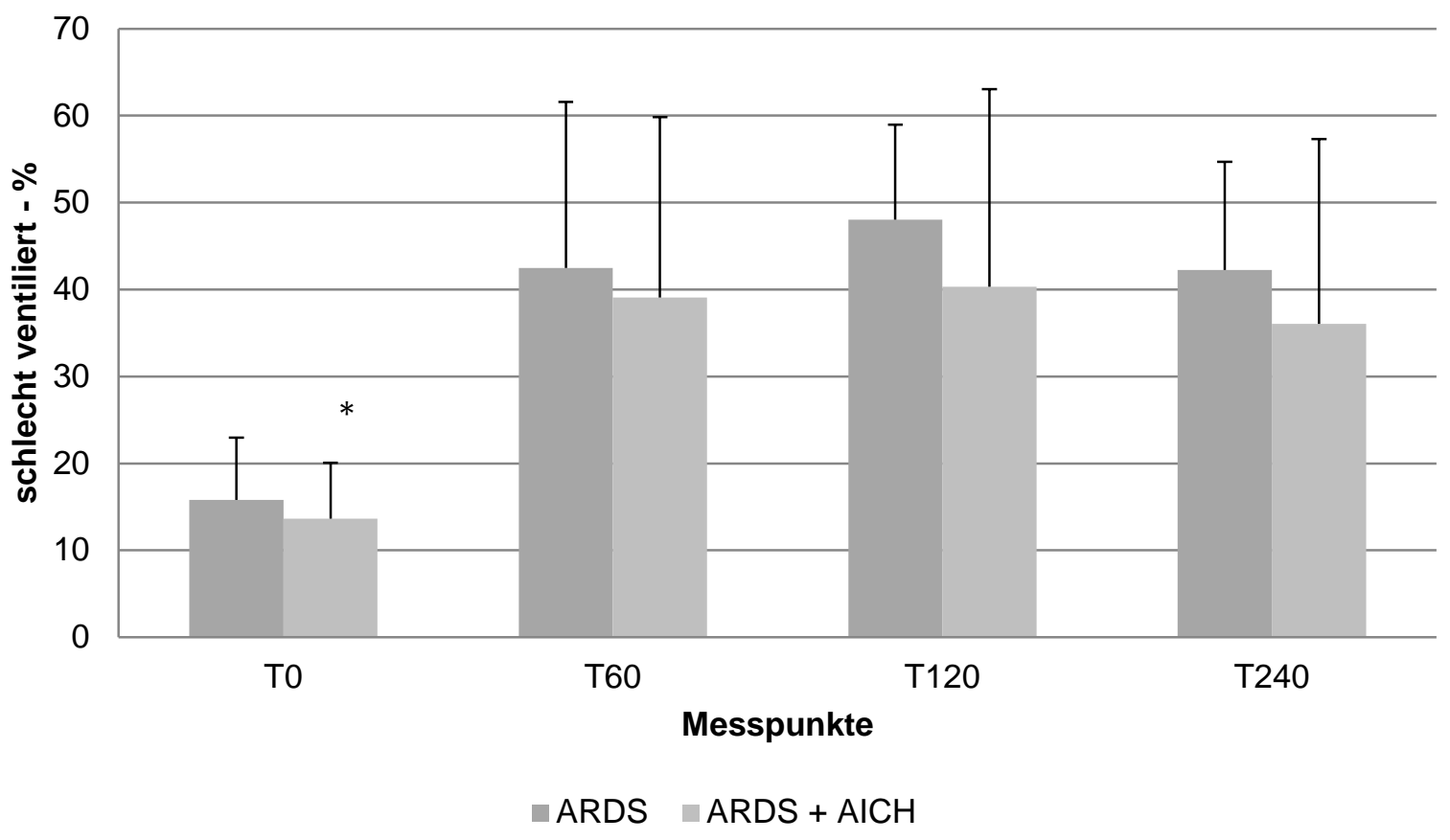

Entwicklung schlecht ventilierter Lungenbereiche für die Gruppen ARDS und ARDS + AlCH über 240 Minuten. Ein signifikanter Unterschied bezüglich schlecht belüfteter Areale fand sich lediglich zum Messpunkt $\left.T_{0} .{ }^{*}=p<0,05\right)$ 
Abbildung 37: Nicht ventilierte Lunge - Control vs. AICH

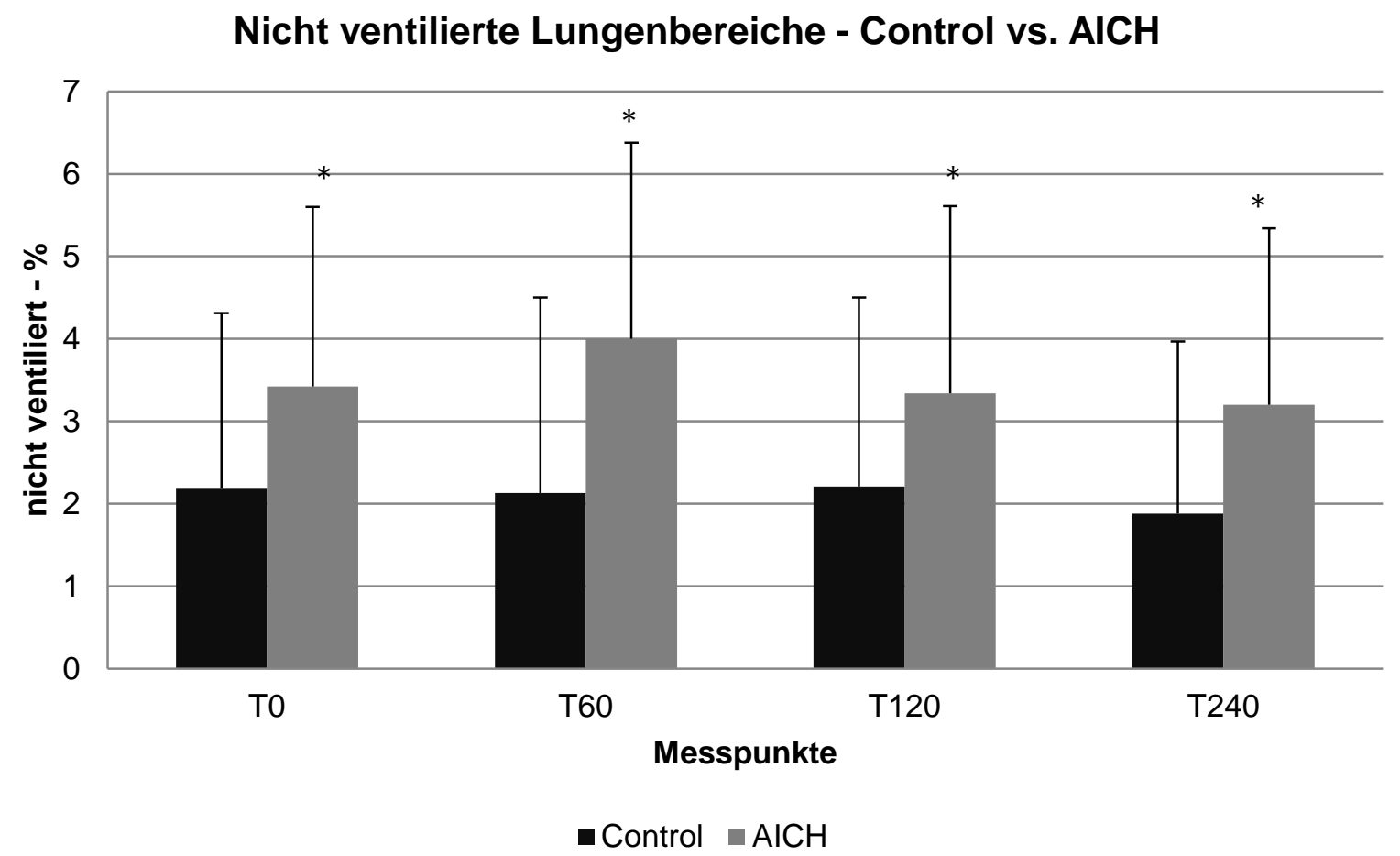

Entwicklung schlecht ventilierter Lungenbereiche für die Gruppen Control und AICH über 240 Minuten. Signifikante Unterschiede finden sich zu allen Messpunkten. Der prozentuale Anteil nicht belüfteter Lungenareale war in der Kontrollgruppe stets geringer als in der AlCH-Gruppe. $\left({ }^{*}=p<0,05\right)$ 
Abbildung 38: Nicht ventilierte Lunge - ARDS vs. ARDS + AICH

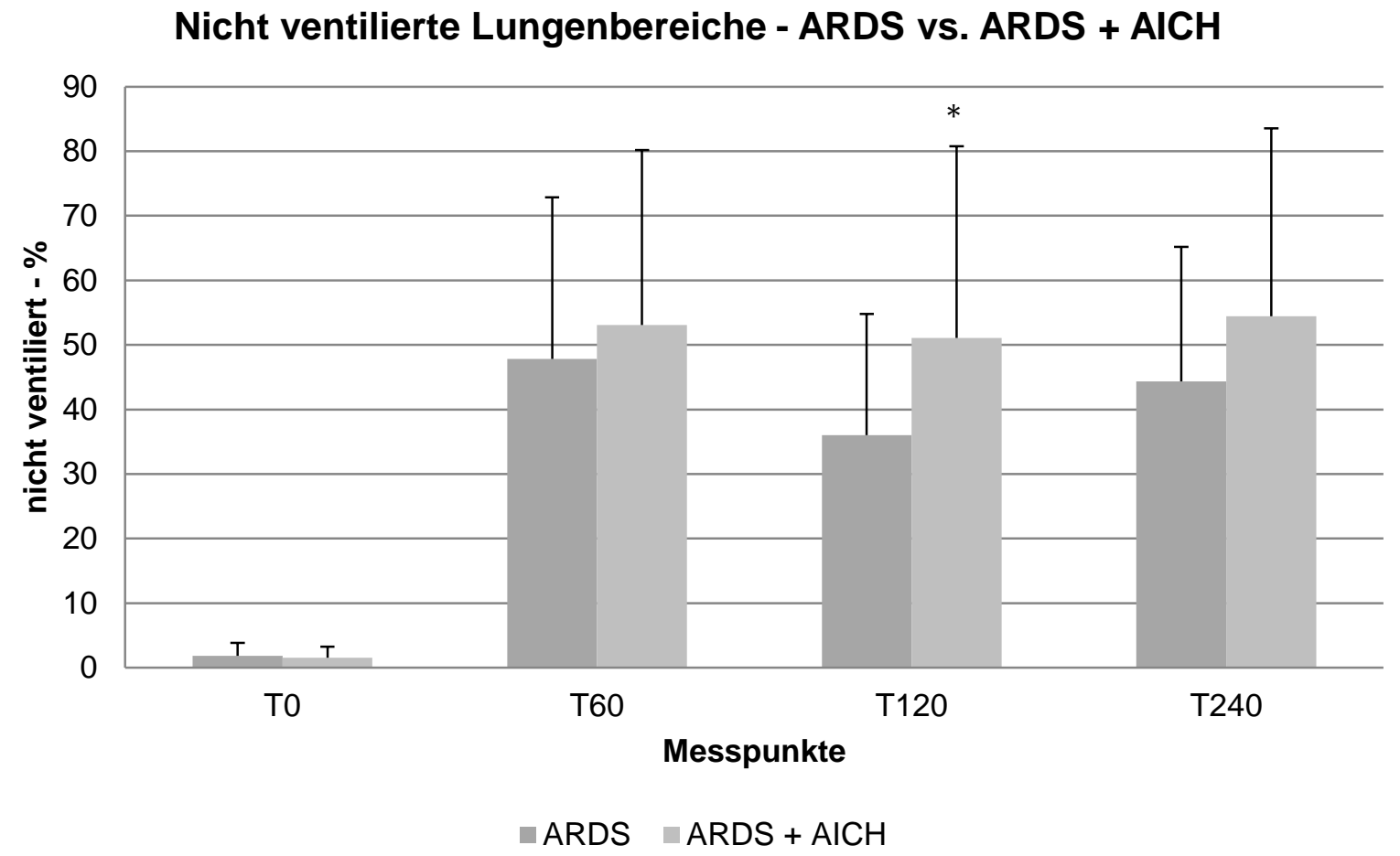

Entwicklung nicht ventilierter Lungenbereiche für die Gruppen ARDS und ARDS + AlCH über 240 Minuten. Signifikante Unterschiede gab es zum Messpunkt T120. Trotz anfänglich weniger nicht belüfteter Lungenbereiche in der Gruppe ARDS + AlCH kommt es hier zu einer vermehrten, wenn auch nicht signifikanten, aber erkennbaren Zunahme nicht belüfteter Areale im Vergleich zur ARDS-Gruppe. $\left(^{*}=\right.$ $p<0,05)$ 


\subsubsection{Segmentanalyse der Lunge}

Der folgende Abschnitt befasst sich mit den Ergebnissen der regionalen Verteilungsanalyse von Hounsfield Units, der Entwicklung des Gasgehaltes in den 10 Segmenten und dem segmentalen Verhältnis von normal, schlecht und nicht ventilierten Lungenbereichen.

Die Ergebnisse der regionalen Lungenparenchymdichtemessung in Hounsfield Units sind in Tabelle 10 tabellarisch dargestellt. Tabelle 11 zeigt die Ergebnisse der Bestimmung des Gasgehalts, die Tabellen 12, 13 und 14 die Entwicklung in den normal, schlecht und nicht ventilierten Bereichen über den gesamten Versuchszeitraum. Die Abbildungen 39 und 40 zeigen den Unterschied $\left(T_{240}-T_{0}\right)$ der mittleren Lungendichte in $\mathrm{HU}$ einmal zwischen der Kontroll- und der AICH-Gruppe sowie zwischen der ARDSund ARDS + AICH-Gruppe von nicht-abhängigen zu abhängigen Lungenarealen.

Die Einteilung erfolgte entlang der vertikalen Achse in 10 Segmente. Die Analyse dieser 10 Segmente fand stets von dorsal nach ventral bzw. von abhängigen zu nichtabhängigen Lungensegmenten statt (siehe Abbildungen $20+21$ ).

Die mittlere Lungendichte war signifikant $(p<0,05)$ unterschiedlich zwischen den Gruppen Control und $\mathrm{AICH}$ in den Segmenten 1,3,5,6,9,10 zum Zeitpunkt $\mathrm{T}_{60}$ und in den Segmenten 3,9,10 zum Zeitpunkt $T_{240}$. Im direkten Vergleich der Gruppen ARDS und $\mathrm{AICH}+$ ARDS konnte eine signifikante $(p<0,05)$ Zunahme der mittleren Lungendichte in den Segmenten 9 und 10 zu den Zeitpunkten $T_{60}$ und $T_{120}$ beobachtet werden (siehe Tabelle 9).

Betrachtet man die mittleren Hounsfield Units über das gesamte Untersuchungsintervall $\left(T_{240}-T_{0}\right)$, lässt sich feststellen, dass es in allen Segmenten der Kontrollgruppe zu einer Abnahme und in der AICH-Gruppe zu einer Zunahme kam. Die mittleren Hounsfield Units in allen Segmenten der ARDS und ARDS + AICH-Gruppe nahmen zu, wobei der Anstieg bei der Kombinationsschädigung ausgeprägter war.

Der Gasgehalt ( $\mathrm{ml}$ Gas/g Gewebe) war im Vergleich signifikant $(p<0,05)$ höher zwischen der Kontroll-Gruppe und der AICH-Gruppe in den Segmenten 1,4,7,8,9 zum Zeitpunkt $T_{60}$ und in den Segmenten 9 und 10 zum Zeitpunkt $T_{240}$.

Der prozentuale Anteil nicht und schlecht belüfteter Lungenareale zum Zeitpunkt $T_{60}$ war signifikant $(p<0,05)$ höher in den Segmenten 1-9 und zum Zeitpunkt $T_{240}$ in den Segmenten 2,3,5,6,7,8 in der Gruppe AICH, verglichen mit der Kontrollgruppe. Außerdem fand sich ein signifikanter $(p<0,05)$ Unterschied im prozentualen Anteil normal ventilierter Lungenareale in den Segmenten 3-8 zum Zeitpunkt $T_{120}$. In der Gruppe 
$\mathrm{AICH}+\mathrm{ARDS}$ änderte sich der Anteil nicht und schlecht belüfteter Lungenareale im Segment 10 zum Zeitpunkt $T_{60}$, in den Segmenten 9 und 10 zum Zeitpunkt $T_{120}$ und im Segment 10 zum Zeitpunkt $T_{240}$ im Vergleich zur ARDS-Gruppe signifikant $(p<0,05)$.

Die Darstellungen der Messwerte in den Tabellen 10 - 14 erfolgen als Mittelwerte mit Standardabweichung von je 7 Tieren pro Gruppe. Control: Kontrollgruppe; AICH: Acute Intracranial Hypertension; ARDS: Acute Respiratory Distress Syndrome; AICH + ARDS: Acute Intracranial Hypertension + Acute Respiratory Distress Syndrome. ${ }^{\circ} p<0,05$ vs. Control; ^ $p<0,05$ vs. ARDS. 
Tabelle 10: Hounsfield Units - Segmente 1 - 10

\begin{tabular}{|c|c|c|c|c|}
\hline 3.4 .3 & Control & $\mathrm{AlCH}$ & ARDS & $\mathrm{ARDS}+\mathrm{AICH}$ \\
\hline \multicolumn{5}{|l|}{$\mathrm{HU}-\mathrm{T}_{0}$} \\
\hline Seg 1 & $-696 \pm 81$ & $-666 \pm 43$ & $-650 \pm 56$ & $-685 \pm 65$ \\
\hline Seg 2 & $-702 \pm 78$ & $-675 \pm 27$ & $-675 \pm 51$ & $-711 \pm 60$ \\
\hline Seg 3 & $-686 \pm 79$ & $-653 \pm 30$ & $-661 \pm 44$ & $-696 \pm 63$ \\
\hline Seg 4 & $-653 \pm 73$ & $-623 \pm 33$ & $-631 \pm 32$ & $-669 \pm 56$ \\
\hline Seg 5 & $-664 \pm 81$ & $-634 \pm 24$ & $-644 \pm 36$ & $-667 \pm 52$ \\
\hline Seg 6 & $-668 \pm 68$ & $-640 \pm 15$ & $-641 \pm 33$ & $-664 \pm 53$ \\
\hline $\operatorname{Seg} 7$ & $-680 \pm 70$ & $-652 \pm 14$ & $-647 \pm 36$ & $-677 \pm 54$ \\
\hline Seg 8 & $-675 \pm 73$ & $-645 \pm 13$ & $-632 \pm 35$ & $-664 \pm 56$ \\
\hline Seg 9 & $-667 \pm 71$ & $-638 \pm 14$ & $-618 \pm 31$ & $-642 \pm 50$ \\
\hline Seg 10 & $-637 \pm 74$ & $-605 \pm 21$ & $-563 \pm 29$ & $-589 \pm 58$ \\
\hline \multicolumn{5}{|c|}{$\mathrm{HU}-\mathrm{T}_{60}$} \\
\hline Seg 1 & $-707 \pm 58$ & $-625 \pm 66^{\circ}$ & $-360 \pm 91$ & $-354 \pm 86$ \\
\hline Seg 2 & $-715 \pm 62$ & $-637 \pm 53$ & $-307 \pm 78$ & $-287 \pm 92$ \\
\hline Seg 3 & $-699 \pm 64$ & $-611 \pm 62^{\circ}$ & $-257 \pm 75$ & $-233 \pm 100$ \\
\hline Seg 4 & $-656 \pm 55$ & $-585 \pm 54$ & $-216 \pm 67$ & $-196 \pm 97$ \\
\hline Seg 5 & $-673 \pm 61$ & $-589 \pm 55^{\circ}$ & $-177 \pm 65$ & $-162 \pm 98$ \\
\hline Seg 6 & $-676 \pm 49$ & $-599 \pm 51^{\circ}$ & $-122 \pm 49$ & $-115 \pm 100$ \\
\hline Seg 7 & $-686 \pm 48$ & $-609 \pm 55$ & $-88 \pm 47$ & $-83 \pm 90$ \\
\hline Seg 8 & $-678 \pm 51$ & $-606 \pm 48$ & $-78 \pm 40$ & $-54 \pm 67$ \\
\hline Seg 9 & $-669 \pm 45$ & $-600 \pm 46^{\circ}$ & $-81 \pm 41$ & $-32 \pm 42^{\wedge}$ \\
\hline Seg 10 & $-629 \pm 39$ & $-565 \pm 46^{\circ}$ & $-95 \pm 53$ & $-25 \pm 26^{\wedge}$ \\
\hline \multicolumn{5}{|c|}{$\mathrm{HU}-\mathrm{T}_{120}$} \\
\hline Seg 1 & $-714 \pm 60$ & $-650 \pm 60$ & $-408 \pm 97$ & $-386 \pm 66$ \\
\hline Seg 2 & $-717 \pm 62$ & $-661 \pm 54$ & $-357 \pm 90$ & $-309 \pm 71$ \\
\hline Seg 3 & $-700 \pm 64$ & $-638 \pm 62$ & $-312 \pm 91$ & $-254 \pm 76$ \\
\hline Seg 4 & $-658 \pm 66$ & $-606 \pm 51$ & $-274 \pm 99$ & $-215 \pm 81$ \\
\hline Seg 5 & $-673 \pm 61$ & $-615 \pm 60$ & $-238 \pm 115$ & $-178 \pm 82$ \\
\hline Seg 6 & $-672 \pm 55$ & $-622 \pm 61$ & $-191 \pm 124$ & $-124 \pm 71$ \\
\hline $\operatorname{Seg} 7$ & $-686 \pm 48$ & $-631 \pm 69$ & $-161 \pm 129$ & $-91 \pm 49$ \\
\hline Seg 8 & $-683 \pm 48$ & $-624 \pm 66$ & $-149 \pm 130$ & $-55 \pm 30$ \\
\hline Seg 9 & $-675 \pm 42$ & $-615 \pm 63$ & $-153 \pm 126$ & $-30 \pm 18$ \\
\hline Seg 10 & $-637 \pm 47$ & $-581 \pm 64$ & $-166 \pm 124$ & $-19 \pm 12$ \\
\hline \multicolumn{5}{|c|}{$\mathrm{HU}-\mathrm{T}_{240}$} \\
\hline Seg 1 & $-740 \pm 77$ & $-640 \pm 45$ & $-397 \pm 118$ & $-385 \pm 82$ \\
\hline $\operatorname{Seg} 2$ & $-746 \pm 75$ & $-656 \pm 34$ & $-322 \pm 118$ & $-302 \pm 76$ \\
\hline Seg 3 & $-734 \pm 75$ & $-635 \pm 36^{\circ}$ & $-266 \pm 119$ & $-240 \pm 60$ \\
\hline Seg 4 & $-689 \pm 82$ & $-605 \pm 33$ & $-230 \pm 109$ & $-202 \pm 63$ \\
\hline Seg 5 & $-704 \pm 76$ & $-608 \pm 34$ & $-201 \pm 109$ & $-150 \pm 64$ \\
\hline Seg 6 & $-705 \pm 75$ & $-614 \pm 30$ & $-163 \pm 107$ & $-103 \pm 68$ \\
\hline Seg 7 & $-717 \pm 69$ & $-621 \pm 34$ & $-141 \pm 105$ & $-75 \pm 60$ \\
\hline Seg 8 & $-713 \pm 70$ & $-613 \pm 29$ & $-116 \pm 94$ & $-52 \pm 45$ \\
\hline Seg 9 & $-696 \pm 60$ & $-602 \pm 28^{\circ}$ & $-104 \pm 80$ & $-36 \pm 31^{\wedge}$ \\
\hline Seg 10 & $-662 \pm 61$ & $-554 \pm 41^{\circ}$ & $-106 \pm 76$ & $-31 \pm 17^{\wedge}$ \\
\hline
\end{tabular}


Tabelle 11: Gasgehalt ml/g - Segmente 1 - 10

\begin{tabular}{|c|c|c|c|c|}
\hline & Control & $\mathrm{AlCH}$ & ARDS & $\mathrm{ARDS}+\mathrm{AICH}$ \\
\hline \multicolumn{5}{|c|}{ Gasgehalt $\mathrm{ml} / \mathrm{g}-$} \\
\hline Seg 1 & $2,46 \pm 0,8$ & $2,09 \pm 0,31$ & $1,85 \pm 0,4$ & $2,22 \pm 0,68$ \\
\hline Seg 2 & $\mid 2,58 \pm 0,82$ & $2,18 \pm 0,23$ & $2,09 \pm 0,39$ & $2,53 \pm 0,75$ \\
\hline Seg 3 & $2,55 \pm 0,79$ & $2,17 \pm 0,21$ & $2,11 \pm 0,34$ & $2,52 \pm 0,71$ \\
\hline Seg 4 & $\mid 2,46 \pm 0,73$ & $2,11 \pm 0,19$ & $2,03 \pm 0,28$ & $2,41 \pm 0,64$ \\
\hline Seg 5 & $2,37 \pm 0,71$ & $2,03 \pm 0,17$ & $1,96 \pm 0,27$ & $2,28 \pm 0,6$ \\
\hline Seg 6 & $\mid 2,37 \pm 0,7$ & $2,02 \pm 0,16$ & $1,93 \pm 0,25$ & $2,24 \pm 0,62$ \\
\hline Seg 7 & $2,36 \pm 0,68$ & $2,02 \pm 0,14$ & $1,87 \pm 0,24$ & $2,19 \pm 0,62$ \\
\hline Seg 8 & | 2,3 $\pm 0,66$ & $1,99 \pm 0,1$ & $1,77 \pm 0,22$ & $2,09 \pm 0,59$ \\
\hline Seg 9 & $2,24 \pm 0,65$ & $1,96 \pm 0,11$ & $1,67 \pm 0,2$ & $1,9 \pm 0,47$ \\
\hline Seg 10 & $\mid 1,93 \pm 0,59$ & $1,67 \pm 0,11$ & $1,25 \pm 0,16$ & $1,45 \pm 0,33$ \\
\hline \multicolumn{5}{|c|}{ Gasgehalt ml/g - } \\
\hline Seg 1 & $\mid 2,44 \pm 0,59$ & $1,82 \pm 0,37^{\circ}$ & $0,79 \pm 0,28$ & $0,66 \pm 0,18$ \\
\hline Seg 2 & $2,66 \pm 0,7$ & $1,9 \pm 0,33$ & $0,72 \pm 0,27$ & $0,54 \pm 0,17$ \\
\hline Seg 3 & $\mid 2,64 \pm 0,68$ & $1,89 \pm 0,34$ & $0,64 \pm 0,25$ & $0,47 \pm 0,17$ \\
\hline Seg 4 & $2,51 \pm 0,59$ & $1,83 \pm 0,32^{\circ}$ & $0,59 \pm 0,25$ & $0,45 \pm 0,17$ \\
\hline Seg 5 & $\mid 2,44 \pm 0,57$ & $1,76 \pm 0,3$ & $0,55 \pm 0,25$ & $0,4 \pm 0,16$ \\
\hline Seg 6 & $2,43 \pm 0,54$ & $1,76 \pm 0,3$ & $0,49 \pm 0,25$ & $0,35 \pm 0,14$ \\
\hline Seg 7 & $\mid 2,38 \pm 0,47$ & $1,77 \pm 0,3^{\circ}$ & $0,48 \pm 0,25$ & $0,32 \pm 0,12$ \\
\hline Seg 8 & $2,29 \pm 0,42$ & $1,74 \pm 0,25^{\circ}$ & $0,46 \pm 0,22$ & $0,31 \pm 0,09$ \\
\hline Seg 9 & $\mid 2,21 \pm 0,4$ & $1,73 \pm 0,22^{\circ}$ & $0,45 \pm 0,21$ & $0,32 \pm 0,11$ \\
\hline Seg 10 & $1,77 \pm 0,29$ & $1,47 \pm 0,2$ & $0,37 \pm 0,14$ & $0,25 \pm 0,05$ \\
\hline \multicolumn{5}{|c|}{ Gasgehalt ml/g - } \\
\hline Seg 1 & $2,52 \pm 0,67$ & $1,98 \pm 0,4$ & $0,99 \pm 0,41$ & $0,73 \pm 0,19$ \\
\hline Seg 2 & $2,69 \pm 0,7$ & $2,09 \pm 0,39$ & $0,89 \pm 0,41$ & $0,59 \pm 0,16$ \\
\hline Seg 3 & $2,67 \pm 0,68$ & $2,06 \pm 0,4$ & $0,8 \pm 0,4$ & $0,51 \pm 0,15$ \\
\hline Seg 4 & $\mid 2,56 \pm 0,64$ & $2 \pm 0,36$ & $0,75 \pm 0,41$ & $0,47 \pm 0,15$ \\
\hline Seg 5 & $2,46 \pm 0,59$ & $1,93 \pm 0,37$ & $0,71 \pm 0,44$ & $0,43 \pm 0,15$ \\
\hline Seg 6 & $\mid 2,45 \pm 0,54$ & $1,94 \pm 0,38$ & $0,64 \pm 0,38$ & $0,37 \pm 0,13$ \\
\hline Seg 7 & $2,4 \pm 0,49$ & $1,93 \pm 0,41$ & $0,63 \pm 0,43$ & $0,33 \pm 0,11$ \\
\hline Seg 8 & $\mid 2,32 \pm 0,45$ & $1,9 \pm 0,4$ & $0,61 \pm 0,39$ & $0,31 \pm 0,1$ \\
\hline Seg 9 & $2,24 \pm 0,46$ & $1,88 \pm 0,37$ & $0,61 \pm 0,34$ & $0,3 \pm 0,07$ \\
\hline Seg 10 & $\mid 1,82 \pm 0,4$ & $1,57 \pm 0,33$ & $0,55 \pm 0,29$ & $0,27 \pm 0,07$ \\
\hline \multicolumn{5}{|c|}{ Gasgehalt ml/g - } \\
\hline Seg 1 & $\mid 3,01 \pm 1$ & $2,02 \pm 0,37$ & $0,96 \pm 0,46$ & $0,79 \pm 0,3$ \\
\hline Seg 2 & $3,22 \pm 1,04$ & $2,15 \pm 0,35$ & $0,85 \pm 0,45$ & $0,61 \pm 0,25$ \\
\hline Seg 3 & $\mid 3,17 \pm 0,98$ & $2,13 \pm 0,36$ & $0,76 \pm 0,43$ & $0,52 \pm 0,21$ \\
\hline Seg 4 & $3,02 \pm 0,91$ & $2,07 \pm 0,37$ & $0,69 \pm 0,4$ & $0,48 \pm 0,19$ \\
\hline Seg 5 & $\mid 2,92 \pm 0,87$ & $1,98 \pm 0,34$ & $0,65 \pm 0,4$ & $0,42 \pm 0,19$ \\
\hline Seg 6 & $2,92 \pm 0,85$ & $1,97 \pm 0,36$ & $0,59 \pm 0,37$ & $0,36 \pm 0,18$ \\
\hline Seg 7 & $\mid 2,87 \pm 0,82$ & $1,94 \pm 0,37$ & $0,57 \pm 0,36$ & $0,33 \pm 0,18$ \\
\hline Seg 8 & $2,78 \pm 0,77$ & $1,87 \pm 0,37$ & $0,53 \pm 0,32$ & $0,33 \pm 0,16$ \\
\hline Seg 9 & $\mid 2,59 \pm 0,68$ & $1,81 \pm 0,39^{\circ}$ & $0,51 \pm 0,29$ & $0,35 \pm 0,16$ \\
\hline Seg 10 & $2,13 \pm 0,56$ & $1,49 \pm 0,36^{\circ}$ & $0,45 \pm 0,25$ & $0,33 \pm 0,13$ \\
\hline
\end{tabular}


Tabelle 12: Normal ventilierte Lungenareale \% - Segmente 1 - 10

\begin{tabular}{|c|c|c|c|c|}
\hline & Control & $\mathrm{AICH}$ & ARDS & $\mathrm{ARDS}+\mathrm{AICH}$ \\
\hline \multicolumn{5}{|c|}{ Normal ventilierte Lungenareale $\%-\mathrm{T}_{0}$} \\
\hline Seg 1 & $84,5 \pm 4,9$ & $82,1 \pm 4,9$ & $82,9 \pm 6,6$ & $83,7 \pm 5$ \\
\hline Seg 2 & $88,3 \pm 4,2$ & $86,3 \pm 3,2$ & $88,5 \pm 6,5$ & $90,3 \pm 4,1$ \\
\hline Seg 3 & $85,7 \pm 4,6$ & $82,8 \pm 3,9$ & $85,7 \pm 5$ & $88 \pm 4,9$ \\
\hline Seg 4 & $79,1 \pm 3,5$ & $77,3 \pm 4,1$ & $79,2 \pm 2,2$ & $82 \pm 4,2$ \\
\hline Seg 5 & $83,3 \pm 4,8$ & $81,4 \pm 2,6$ & $84,8 \pm 2,9$ & $84,7 \pm 3,3$ \\
\hline Seg 6 & $85,1 \pm 3$ & $83,3 \pm 1,9$ & $84,9 \pm 2,9$ & $85,6 \pm 4$ \\
\hline $\operatorname{Seg} 7$ & $86,8 \pm 3,2$ & $85,4 \pm 2,1$ & $85 \pm 3,4$ & $87,4 \pm 4,9$ \\
\hline Seg 8 & $85,1 \pm 4,5$ & $83,7 \pm 2,2$ & $82,2 \pm 3,7$ & $85,1 \pm 5,5$ \\
\hline Seg 9 & $82,8 \pm 5,5$ & $81,5 \pm 2,4$ & $79 \pm 3,4$ & $80,9 \pm 5,4$ \\
\hline Seg 10 & $74,5 \pm 8,2$ & $73,1 \pm 3$ & $65 \pm 3,3$ & $69,4 \pm 9,6$ \\
\hline \multicolumn{5}{|c|}{ Normal ventilierte Lungenareale $\%-\mathrm{T}_{60}$} \\
\hline Seg 1 & $85,4 \pm 3,1$ & $77,5 \pm 8,9$ & $25,4 \pm 15,5$ & $25,9 \pm 13,5$ \\
\hline Seg 2 & $89,4 \pm 3,3$ & $82,7 \pm 5,9$ & $16,6 \pm 11,1$ & $14,4 \pm 10,3$ \\
\hline Seg 3 & $86,9 \pm 3,8$ & $78,3 \pm 7,1$ & $11,8 \pm 7,8$ & $9,1 \pm 8,2$ \\
\hline Seg 4 & $78,9 \pm 2,1$ & $73,4 \pm 6,2$ & $9,5 \pm 6,4$ & $7,6 \pm 6,7$ \\
\hline Seg 5 & $83,9 \pm 3,3$ & $76,3 \pm 6,2$ & $7,6 \pm 4,9$ & $5,3 \pm 4,7$ \\
\hline Seg 6 & $85,6 \pm 1,9$ & $78,7 \pm 5,8$ & $5,1 \pm 3,6$ & $3,1 \pm 3,1$ \\
\hline Seg 7 & $86,9 \pm 2,2$ & $80,1 \pm 6,4$ & $4,4 \pm 3,7$ & $2,5 \pm 2,3$ \\
\hline Seg 8 & $85,3 \pm 3,5$ & $79 \pm 5,8$ & $4,7 \pm 3,2$ & $2,3 \pm 1,7$ \\
\hline Seg 9 & $82,4 \pm 2,5$ & $77 \pm 6,8$ & $4,8 \pm 2,7$ & $2,1 \pm 1,4$ \\
\hline Seg 10 & $71,5 \pm 2,5$ & $67,6 \pm 7$ & $4,2 \pm 2,5$ & $1,7 \pm 0,6$ \\
\hline \multicolumn{5}{|c|}{ Normal ventilierte Lungenareale $\%-\mathrm{T}_{120}$} \\
\hline Seg 1 & $85,8 \pm 2,9$ & $81,1 \pm 7$ & $33,8 \pm 19,9$ & $29,8 \pm 12,5$ \\
\hline Seg 2 & $89,5 \pm 2,4$ & $85 \pm 5,1$ & $24,5 \pm 17,5$ & $15,9 \pm 9,2$ \\
\hline Seg 3 & $86,6 \pm 2,9$ & $81,2 \pm 6^{\circ}$ & $19,8 \pm 15,4$ & $9,9 \pm 5,7$ \\
\hline Seg 4 & $78,8 \pm 3,3$ & $75,3 \pm 4,7^{\circ}$ & $17,1 \pm 14,6$ & $7,7 \pm 4,8$ \\
\hline Seg 5 & $83,7 \pm 2,4$ & $79,1 \pm 5,9^{\circ}$ & $14,1 \pm 13,3$ & $5,6 \pm 3,4$ \\
\hline Seg 6 & $84,8 \pm 2,6$ & $80,9 \pm 6,6^{\circ}$ & $10,9 \pm 11,6$ & $3,3 \pm 2,5$ \\
\hline $\operatorname{Seg} 7$ & $86,7 \pm 1,5$ & $81,8 \pm 8,3^{\circ}$ & $8,6 \pm 9,8$ & $2,4 \pm 1,8$ \\
\hline Seg 8 & $85,7 \pm 2$ & $79,8 \pm 9,2^{\circ}$ & $8,2 \pm 8,3$ & $1,9 \pm 1,1$ \\
\hline Seg 9 & $82,8 \pm 2,1$ & $77,7 \pm 10,1$ & $8,5 \pm 7,6$ & $2 \pm 1$ \\
\hline Seg 10 & $72,8 \pm 3,8$ & $69,1 \pm 10,2$ & $8,4 \pm 7,5$ & $2 \pm 1,1$ \\
\hline \multicolumn{5}{|c|}{ Normal ventilierte Lungenareale $\%-\mathrm{T}_{240}$} \\
\hline Seg 1 & $85,7 \pm 2,9$ & $82,4 \pm 6,1$ & $34,1 \pm 23,1$ & $31,6 \pm 15,2$ \\
\hline Seg 2 & $89,4 \pm 2$ & $86,2 \pm 4,3$ & $22,8 \pm 17,3$ & $17,6 \pm 11,1$ \\
\hline Seg 3 & $87,5 \pm 1,7$ & $82,7 \pm 4$ & $16,4 \pm 13,7$ & $10,8 \pm 7,7$ \\
\hline Seg 4 & $79,4 \pm 2,6$ & $76,9 \pm 3,9$ & $13,6 \pm 11,7$ & $8,6 \pm 6,1$ \\
\hline Seg 5 & $84 \pm 2,3$ & $80,3 \pm 4,4$ & $10,9 \pm 9,9$ & $6 \pm 4,6$ \\
\hline Seg 6 & $85,4 \pm 2,1$ & $81,7 \pm 4,5$ & $8,3 \pm 8,2$ & $3,9 \pm 4,2$ \\
\hline Seg 7 & $87,3 \pm 2$ & $82,7 \pm 5,9$ & $7,2 \pm 7,1$ & $2,9 \pm 3,2$ \\
\hline Seg 8 & $86,8 \pm 2,9$ & $80,3 \pm 6,7$ & $6,3 \pm 5,8$ & $2,4 \pm 2,1$ \\
\hline Seg 9 & $84,1 \pm 3,7$ & $77,4 \pm 7,6$ & $6 \pm 4,9$ & $2,4 \pm 1,7$ \\
\hline Seg 10 & $76,3 \pm 5,9$ & $66 \pm 10,8$ & $5,1 \pm 4$ & $2,3 \pm 1,1$ \\
\hline
\end{tabular}


Tabelle 13: Schlecht ventilierte Lungenareale \% - Segmente 1 - 10

\begin{tabular}{|c|c|c|c|c|}
\hline & Control & $\mathrm{AlCH}$ & ARDS & $\mathrm{ARDS}+\mathrm{AICH}$ \\
\hline \multicolumn{5}{|c|}{ Schlecht ventilierte Lungenareale $\%-\mathrm{T}_{0}$} \\
\hline Seg 1 & $11 \pm 7,5$ & $13,9 \pm 5,2$ & $15,7 \pm 6,6$ & $14,2 \pm 6,4$ \\
\hline Seg 2 & $8,4 \pm 5,7$ & $10,6 \pm 2,9$ & $10,6 \pm 6,2$ & $8,4 \pm 4,7$ \\
\hline Seg 3 & $8,3 \pm 4,7$ & $10,2 \pm 2,1$ & $10,5 \pm 3,9$ & $8,6 \pm 4,2$ \\
\hline $\operatorname{Seg} 4$ & $9,8 \pm 4,8$ & $11,2 \pm 2,1$ & $11,9 \pm 2,2$ & $10,4 \pm 3,9$ \\
\hline Seg 5 & $9,6 \pm 5$ & $11,3 \pm 2,1$ & $11,4 \pm 2,5$ & $10,3 \pm 3,6$ \\
\hline Seg 6 & $9,2 \pm 4,6$ & $11,1 \pm 1,9$ & $11,8 \pm 3,2$ & $10,6 \pm 4,6$ \\
\hline Seg 7 & $9,9 \pm 4,8$ & $11,3 \pm 1,4$ & $14,3 \pm 3,5$ & $11,6 \pm 5,2$ \\
\hline Seg 8 & $11,9 \pm 6$ & $13,3 \pm 1$ & $17,3 \pm 3,7$ & $14,2 \pm 5,6$ \\
\hline Seg 9 & $14,1 \pm 7,1$ & $15,1 \pm 1,6$ & $20,4 \pm 3,3$ & $18,4 \pm 5,6$ \\
\hline Seg 10 & $21,1 \pm 10,3$ & $22,9 \pm 2,4$ & $34 \pm 3,6$ & $29,7 \pm 10$ \\
\hline \multicolumn{5}{|c|}{ Schlecht ventilierte Lungenareale $\%-\mathrm{T}_{60}$} \\
\hline Seg 1 & $10 \pm 4,4$ & $19,2 \pm 9,1^{\circ}$ & $63,2 \pm 7,7$ & $61,1 \pm 8,5$ \\
\hline Seg 2 & $7,4 \pm 4,4$ & $14,1 \pm 5,2^{\circ}$ & $68,2 \pm 8,2$ & $66,2 \pm 8,9$ \\
\hline Seg 3 & $7,5 \pm 3,7$ & $13,5 \pm 4,6^{\circ}$ & $64,3 \pm 9,6$ & $61,7 \pm 11$ \\
\hline Seg 4 & $9,1 \pm 3,4$ & $14,9 \pm 4,6^{\circ}$ & $54,3 \pm 13,3$ & $49,9 \pm 12,7$ \\
\hline Seg 5 & $8,9 \pm 3,4$ & $15,8 \pm 5,2^{\circ}$ & $46,6 \pm 15,5$ & $45 \pm 17,6$ \\
\hline Seg 6 & $8,2 \pm 3,2$ & $15,9 \pm 5,2^{\circ}$ & $34,1 \pm 12,4$ & $36 \pm 24,2$ \\
\hline Seg 7 & $9,8 \pm 3,5$ & $16,4 \pm 5,3^{\circ}$ & $23,7 \pm 9,7$ & $27,5 \pm 24,8$ \\
\hline Seg 8 & $12,1 \pm 4,5$ & $17,7 \pm 4,7^{\circ}$ & $20 \pm 8$ & $19,3 \pm 17,2$ \\
\hline Seg 9 & $14,6 \pm 3,7$ & $19,5 \pm 5,8^{\circ}$ & $21,6 \pm 8,5$ & $13 \pm 9,2$ \\
\hline Seg 10 & $24,3 \pm 4,3$ & $28,5 \pm 5,7$ & $29 \pm 14,5$ & $11,3 \pm 6^{\wedge}$ \\
\hline \multicolumn{5}{|c|}{ Schlecht ventilierte Lungenareale $\%-\mathrm{T}_{120}$} \\
\hline Seg 1 & $9 \pm 4$ & $15,6 \pm 7,4$ & $57,9 \pm 15,4$ & $61 \pm 11$ \\
\hline Seg 2 & $7 \pm 3,8$ & $12 \pm 4,9$ & $64,2 \pm 13,6$ & $69 \pm 10,5$ \\
\hline Seg 3 & $7,3 \pm 3,4$ & $12 \pm 4,7$ & $61,9 \pm 10,2$ & $65,4 \pm 13,3$ \\
\hline Seg 4 & $8,9 \pm 3,6$ & $13,4 \pm 4,7$ & $53,6 \pm 10$ & $53,8 \pm 14,7$ \\
\hline Seg 5 & $9 \pm 3,6$ & $13,8 \pm 5,2$ & $49,4 \pm 13,6$ & $48,2 \pm 19,1$ \\
\hline Seg 6 & $8,6 \pm 3,5$ & $13,8 \pm 5,9$ & $43,3 \pm 18,7$ & $38,7 \pm 21,4$ \\
\hline $\operatorname{Seg} 7$ & $9,4 \pm 3,11$ & $15 \pm 7,5$ & $38,5 \pm 20,8$ & $30,1 \pm 15,6$ \\
\hline Seg 8 & $11,1 \pm 3,6$ & $17,3 \pm 8,9$ & $34,5 \pm 23,7$ & $18,7 \pm 8,5$ \\
\hline Seg 9 & $13,8 \pm 3,7$ & $19,2 \pm 9,6$ & $36,2 \pm 24,6$ & $11 \pm 3,9^{\wedge}$ \\
\hline Seg 10 & $22,3 \pm 6,2$ & $27,4 \pm 9,4$ & $41 \pm 25,1$ & $7,4 \pm 2,5^{\wedge}$ \\
\hline \multicolumn{5}{|c|}{ Schlecht ventilierte Lungenareale $\%-\mathrm{T}_{240}$} \\
\hline Seg 1 & $7,2 \pm 4,3$ & $14,4 \pm 6,9$ & $56,2 \pm 17,6$ & $58,3 \pm 13,6$ \\
\hline Seg 2 & $5,5 \pm 3,7$ & $11,1 \pm 4,3^{\circ}$ & $59,8 \pm 9,9$ & $65 \pm 11,6$ \\
\hline Seg 3 & $5,7 \pm 3,1$ & $10,9 \pm 3,7^{\circ}$ & $55,4 \pm 10,2$ & $61,4 \pm 11$ \\
\hline Seg 4 & $7,6 \pm 3,7$ & $12,2 \pm 3,8$ & $47,4 \pm 8,7$ & $48,2 \pm 7,6$ \\
\hline Seg 5 & $7,4 \pm 3,4$ & $12,6 \pm 4^{\circ}$ & $45,4 \pm 12,1$ & $38,5 \pm 8,8$ \\
\hline Seg 6 & $7,1 \pm 3,9$ & $12,8 \pm 4,3^{\circ}$ & $39,5 \pm 15,5$ & $29,9 \pm 14,6$ \\
\hline $\operatorname{Seg} 7$ & $7,8 \pm 3,8$ & $14,1 \pm 5,3^{\circ}$ & $35 \pm 18,2$ & $23,2 \pm 13,9$ \\
\hline Seg 8 & $9,2 \pm 4,6$ & $16,7 \pm 6,3^{\circ}$ & $29 \pm 17,3$ & $15,8 \pm 9,9$ \\
\hline Seg 9 & $12,2 \pm 4,7$ & $19,5 \pm 7$ & $26 \pm 14,1$ & $10,6 \pm 5,8$ \\
\hline Seg 10 & $19 \pm 7,7$ & $30,8 \pm 10$ & $28,9 \pm 17,4$ & $9,6 \pm 3^{\wedge}$ \\
\hline
\end{tabular}


Tabelle 14: Nicht ventilierte Lungenareale \% - Segmente 1 - 10

\begin{tabular}{|c|c|c|c|c|}
\hline & Control & $\mathrm{AICH}$ & ARDS & $\mathrm{ARDS}+\mathrm{AICH}$ \\
\hline \multicolumn{5}{|c|}{ Nicht ventilierte Lungenareale $\%-\mathrm{T}_{0}$} \\
\hline Seg 1 & $0,3 \pm 0,4$ & $1,2 \pm 1,1$ & $0,6 \pm 0,7$ & $0,2 \pm 0,1$ \\
\hline $\operatorname{Seg} 2$ & $0,8 \pm 0,7$ & $1,5 \pm 0,9$ & $0,6 \pm 0,5$ & $0,3 \pm 0,2$ \\
\hline Seg 3 & $3,2 \pm 1,8$ & $4,9 \pm 2,1$ & $2,9 \pm 1,1$ & $2,2 \pm 1,3$ \\
\hline Seg 4 & $6,6 \pm 1,2$ & $7,9 \pm 2,3$ & $6,3 \pm 1,2$ & $4,7 \pm 1$ \\
\hline Seg 5 & $4,4 \pm 1,5$ & $5,4 \pm 1,2$ & $3,3 \pm 0,8$ & $3,6 \pm 0,5$ \\
\hline Seg 6 & $3,5 \pm 1$ & $4,1 \pm 0,9$ & $3,1 \pm 0,5$ & $3,2 \pm 0,4$ \\
\hline Seg 7 & $1,3 \pm 0,4$ & $2,2 \pm 1,2$ & $0,5 \pm 0,1$ & $0,5 \pm 0,3$ \\
\hline Seg 8 & $0,7 \pm 0,4$ & $1,8 \pm 1,6$ & $0,3 \pm 0,2$ & $0,2 \pm 0,2$ \\
\hline Seg 9 & $0,5 \pm 0,4$ & $1,8 \pm 1,8$ & $0,4 \pm 0,4$ & $0,2 \pm 0,3$ \\
\hline Seg 10 & $0,6 \pm 0,7$ & $1,8 \pm 2,1$ & $0,5 \pm 0,5$ & $0,2 \pm 0,3$ \\
\hline \multicolumn{5}{|c|}{ Nicht ventilierte Lungenareale $\%-T_{60}$} \\
\hline Seg 1 & $0,1 \pm 0,1$ & $1,4 \pm 1,2^{\circ}$ & $11,3 \pm 10,4$ & $12,9 \pm 9,9$ \\
\hline Seg 2 & $0,6 \pm 0,3$ & $2 \pm 1,4^{\circ}$ & $15,1 \pm 11,1$ & $19,2 \pm 12,6$ \\
\hline Seg 3 & $2,7 \pm 1,5$ & $6,4 \pm 3,1^{\circ}$ & $23,7 \pm 14$ & $28,9 \pm 16,5$ \\
\hline Seg 4 & $7,3 \pm 1,2$ & $8,5 \pm 2,6^{\circ}$ & $35,3 \pm 15,9$ & $41,1 \pm 18,1$ \\
\hline Seg 5 & $4,3 \pm 1,3$ & $6,4 \pm 1,8^{\circ}$ & $45,2 \pm 17,8$ & $48,8 \pm 21,4$ \\
\hline Seg 6 & $3,8 \pm 0,9$ & $4,3 \pm 1,5^{\circ}$ & $60,5 \pm 13,7$ & $60,5 \pm 26,8$ \\
\hline Seg 7 & $1,2 \pm 0,3$ & $2,6 \pm 1,9^{\circ}$ & $71,8 \pm 11,7$ & $69,7 \pm 26,6$ \\
\hline Seg 8 & $0,6 \pm 0,2$ & $2,3 \pm 2,3^{\circ}$ & $75,1 \pm 9,6$ & $78,1 \pm 18,5$ \\
\hline Seg 9 & $0,4 \pm 0,1$ & $2,2 \pm 2,5^{\circ}$ & $73,5 \pm 10$ & $84,6 \pm 10,2$ \\
\hline Seg 10 & $0,2 \pm 0,1$ & $2,3 \pm 2,9$ & $66,7 \pm 15,7$ & $86,9 \pm 6,2^{\wedge}$ \\
\hline \multicolumn{5}{|c|}{ Nicht ventilierte Lungenareale $\%-\mathrm{T}_{120}$} \\
\hline Seg 1 & $0,2 \pm 0,3$ & $0,9 \pm 1$ & $7,9 \pm 5,6$ & $9 \pm 7$ \\
\hline Seg 2 & $0,6 \pm 0,4$ & $1,4 \pm 1,1$ & $11 \pm 5,7$ & $14,8 \pm 11,2$ \\
\hline Seg 3 & $2,8 \pm 1,4$ & $4,9 \pm 2,5$ & $18 \pm 8,4$ & $24,4 \pm 16,2$ \\
\hline Seg 4 & $7,2 \pm 1,8$ & $7,8 \pm 2,3$ & $28,1 \pm 12,5$ & $36,8 \pm 18,4$ \\
\hline Seg 5 & $4,1 \pm 0,8$ & $5,4 \pm 1,5$ & $35,4 \pm 19$ & $45,1 \pm 21,6$ \\
\hline Seg 6 & $4 \pm 1,1$ & $3,9 \pm 1,6$ & $45,3 \pm 25$ & $57,6 \pm 22,4$ \\
\hline $\operatorname{Seg} 7$ & $1,5 \pm 0,4$ & $2,1 \pm 1,8$ & $52,6 \pm 27,7$ & $67,2 \pm 16$ \\
\hline Seg 8 & $0,7 \pm 0,3$ & $1,7 \pm 1,9$ & $56,9 \pm 30,3$ & $79 \pm 9$ \\
\hline Seg 9 & $0,5 \pm 0,3$ & $1,7 \pm 2,1$ & $55 \pm 30,9$ & $86,7 \pm 4,7^{\wedge}$ \\
\hline Seg 10 & $0,5 \pm 0,4$ & $1,8 \pm 2,4$ & $50,2 \pm 31,4$ & $90,5 \pm 3,1^{\wedge}$ \\
\hline \multicolumn{5}{|c|}{ Nicht ventilierte Lungenareale $\%-\mathrm{T}_{240}$} \\
\hline Seg 1 & $0,1 \pm 0,1$ & $0,6 \pm 0,4$ & $9,4 \pm 6,8$ & $9,9 \pm 6$ \\
\hline Seg 2 & $0,4 \pm 0,2$ & $1,2 \pm 0,7^{\circ}$ & $17,3 \pm 13,2$ & $17,1 \pm 10,5$ \\
\hline Seg 3 & $2 \pm 1$ & $4,3 \pm 1,5^{\circ}$ & $27,9 \pm 19,2$ & $27,3 \pm 11,2$ \\
\hline Seg 4 & $6,4 \pm 2,1$ & $7,1 \pm 2,2$ & $38 \pm 18,6$ & $41 \pm 10,7$ \\
\hline Seg 5 & $3,8 \pm 0,8$ & $5,3 \pm 1,2^{\circ}$ & $43 \pm 20,8$ & $54,2 \pm 13,6$ \\
\hline Seg 6 & $3,5 \pm 1$ & $4,1 \pm 1,1^{\circ}$ & $51,8 \pm 23$ & $65,7 \pm 18$ \\
\hline Seg 7 & $1,2 \pm 0,6$ & $2,2 \pm 1,2^{\circ}$ & $57,7 \pm 24,6$ & $73,5 \pm 16,4$ \\
\hline Seg 8 & $0,6 \pm 0,3$ & $1,7 \pm 1,4^{\circ}$ & $64,5 \pm 22,8$ & $81,2 \pm 11,9$ \\
\hline Seg 9 & $0,4 \pm 0,3$ & $1,6 \pm 1,6$ & $67,9 \pm 19$ & $86,4 \pm 7,5$ \\
\hline Seg 10 & $0,3 \pm 0,3$ & $1,7 \pm 2$ & $65,9 \pm 20,7$ & $87,9 \pm 3,9^{\wedge}$ \\
\hline
\end{tabular}


Abbildung 39: Delta HU von $T_{240}-T_{0}$-Control vs. AlCH

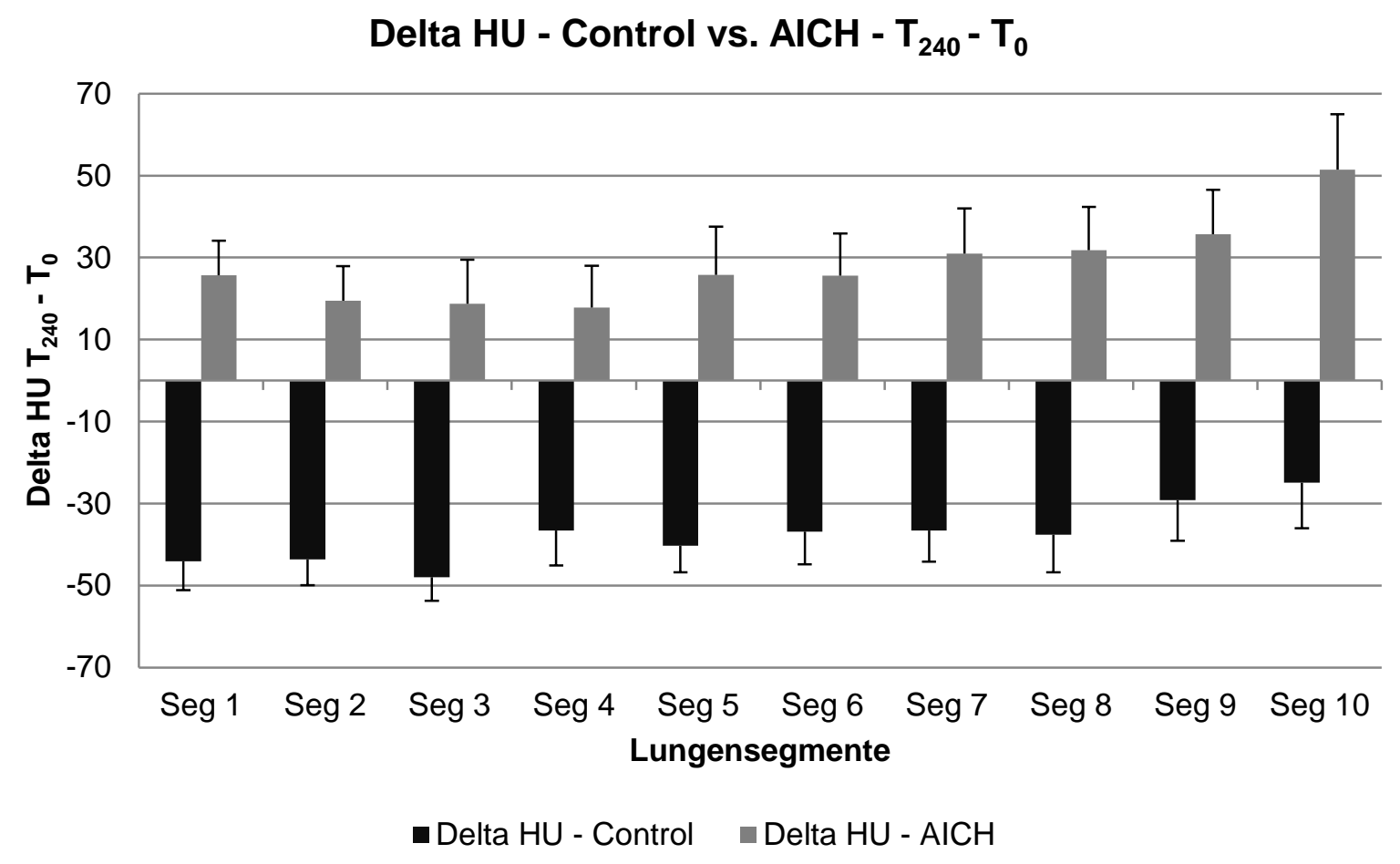

Dargestellt ist die Entwicklung der mittleren Lungenparenchymdichte, bezogen auf den Zeitpunkt $T_{0}$ für die Gruppen Control und AICH. Aufgetragen ist die mittlere Lungenparenchymdichte für jedes der 10 Segmente. Es ist eine Abnahme der Lungenparenchymdichte in der Kontrollgruppe über alle Segmente zu erkennen. In der AlCH-Gruppe kam es in allen Segmenten, wenn auch nicht stets signifikant, zu einer Zunahme der Lungendichte. 
Abbildung 40: Delta HU von $\mathrm{T}_{240}-\mathrm{T}_{0}$ ARDS und ARDS + AICH

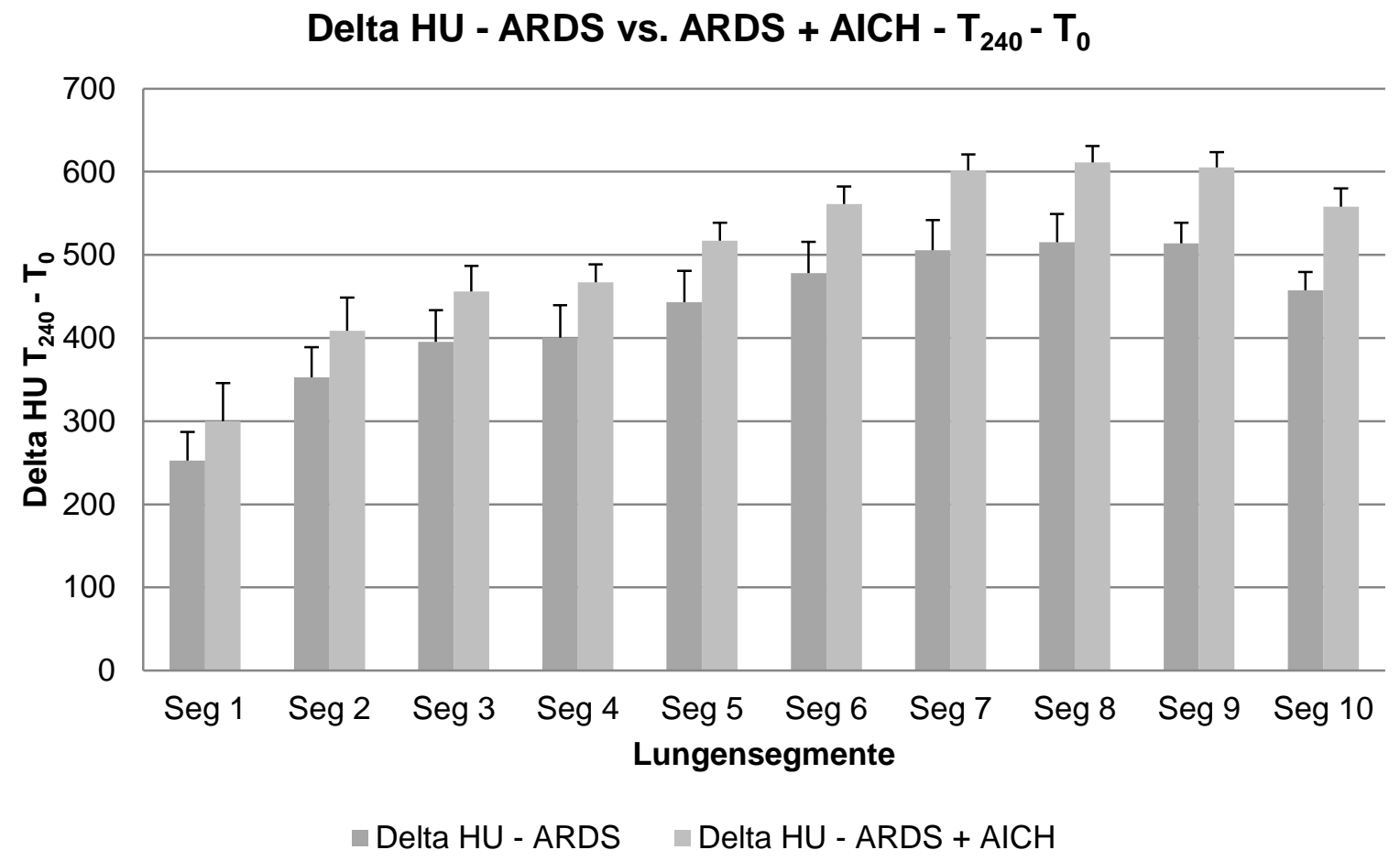

Dargestellt ist die Entwicklung der mittleren Lungenparenchymdichte, bezogen auf den Zeitpunkt $T_{0}$ für die Gruppen ARDS und ARDS + AICH. Aufgetragen ist die mittlere Lungenparenchymdichte für jedes der 10 Segmente. Es ist eine stärkere, wenn auch nicht immer signifikante Zunahme der Lungenparenchymdichte in der $A R D S+A I C H$-Gruppe über alle Segmente zu erkennen. In der ARDS-Gruppe kam es ebenfalls in allen Segmenten zu einer Zunahme der Lungendichte. (Publiziert in Intensive Care Med (2011) 37:1182-1191, Fig. 2) 


\subsection{Extravaskulärer Lungenwasserindex und Hounsfield Units}

Der extravaskuläre Lungenwasserindex (EVLWI) aller Gruppen ist der Tabelle $15 \mathrm{zu}$ entnehmen. Die graphische Darstellung der Ergebnisse als Balkendiagramm mit Standardabweichung ist in Abbildung 41 aufgezeigt. Die Abbildungen 42 - 45 veranschaulichen den Verlauf von Hounsfield Units und extravaskulärem Lungenwasserindex der einzelnen Versuchgruppen anhand eines Liniendiagramms.

Im Vergleich der Gruppen Control und AICH kam es zu einem signifikanten $(p<0,05)$ Anstieg des extravaskulären Lungewasserindex zu den Messpunkten $T_{120}$ und $T_{240}$. Zwischen den Gruppen ARDS und ARDS + AICH gab es signifikante $(p<0,05)$ Unterschiede an den Messpunkten $T_{60}, T_{120}$ und $T_{240}$.

Tabelle 15: Extravaskulärer Lungenwasserindex (EVLWI)

\begin{tabular}{lllll} 
& $\mathbf{T}_{\mathbf{0}}$ & $\mathbf{T}_{\mathbf{6 0}}$ & $\mathbf{T}_{\mathbf{1 2 0}}$ & $\mathbf{T}_{\mathbf{2 4 0}}$ \\
\hline EVLWI (ml/kg) & & & & \\
Control & $8,98 \pm 1,86$ & $8,53 \pm 1,23$ & $8,5 \pm 1,44$ & $8,71 \pm 1,15$ \\
\hline AICH & $8,71 \pm 0,99$ & $9,64 \pm 1,35$ & $9,89 \pm 1,28^{\circ}$ & $10,76 \pm 1,43^{\circ}$ \\
ARDS & $8,14 \pm 1,35$ & $13,43 \pm 2,23^{* \circ}$ & $18,71 \pm 2,14^{* \circ}$ & $18,71 \pm 2,21^{* \circ}$ \\
\hline ARDS + AICH & $7,64 \pm 0,48$ & $18,86 \pm 5,21^{* \circ}$ & $30,57 \pm 10,72^{\star \circ \wedge}$ & $24,29 \pm 7,27^{\star \circ \wedge}$
\end{tabular}

Darstellung als Mittelwerte (EVLWI=Extravaskulärer Lungenwasserindex) mit Standardabweichung von je 7 Tieren pro Gruppe. Control: Kontrollgruppe; AlCH: Acute Intracranial Hypertension; ARDS: Acute Respiratory Distress Syndrome; $\mathrm{AlCH}+$ ARDS: Acute Intracranial Hypertension + Acute Respiratory Distress Syndrome. $p<0,05$ vs. $T_{0} ;{ }^{\circ} p<0,05$ vs. Control: ${ }^{\wedge} p<0,05$ vs. ARDS. 
Abbildung 41: Extravaskulärer Lungenwasserindex (EVLWI) - $T_{0}-T_{240}$

Extravaskulärer Lungenwasserindex (EVLWI)

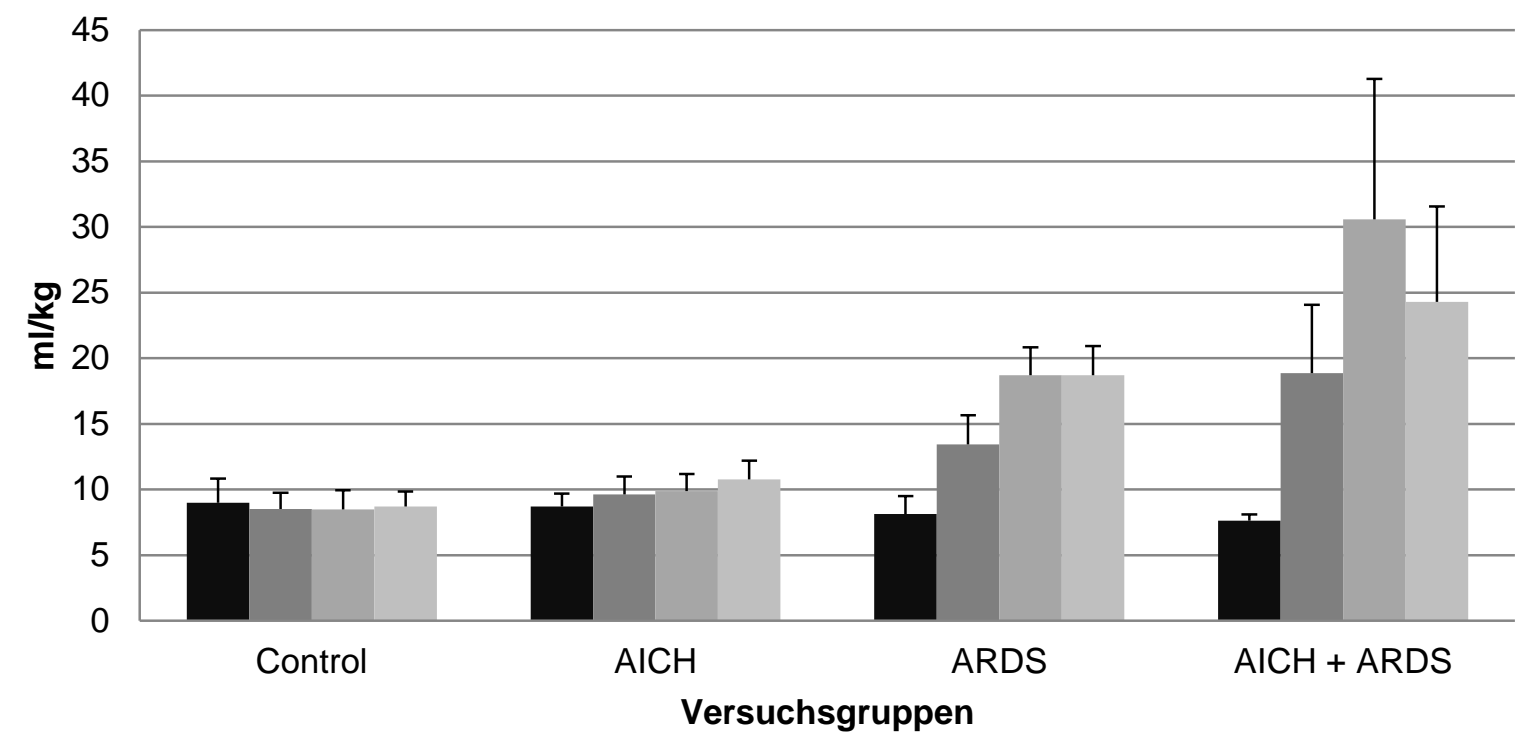

चT0 $\quad$ T60 $\quad$ T120 $\square$ T240

Das Balkendiagramm zeigt den Verlauf des EVLWI für die vier Versuchsgruppen zu allen Messpunkten. Der höchste Anstieg des EVLWI fand sich in der ARDS + AlCHGruppe, gefolgt von der ARDS-Gruppe und dann der AlCH-Gruppe. (Publiziert in Intensive Care Med (2011) 37:1182-1191, Fig. 3) 
Abbildung 42: Entwicklung HU vs. EVLWI - Control - $\mathrm{T}_{0}-\mathrm{T}_{240}$

HU vs. EVLWI - Control

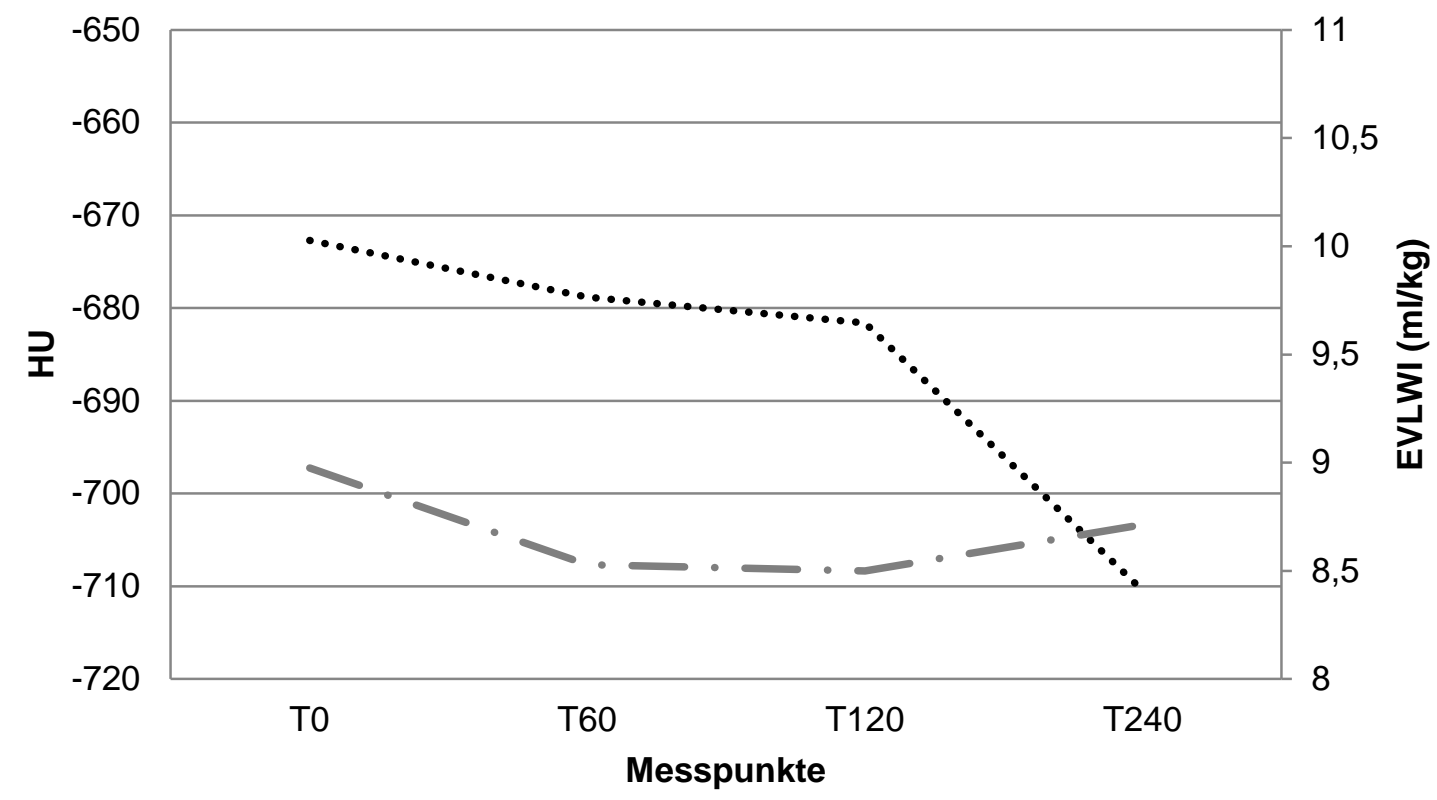

$\mathrm{HU}-\cdot \mathrm{EVLWI}$

Innerhalb der Kontrollgruppe ging die Abnahme des EVLWI auch mit einer Reduktion der Lungenparenchymdichte einher.

Abbildung 43: Entwicklung HU vs. EVLWI - AICH - $T_{0}-T_{240}$

HU vs. EVLWI - AICH Gruppe

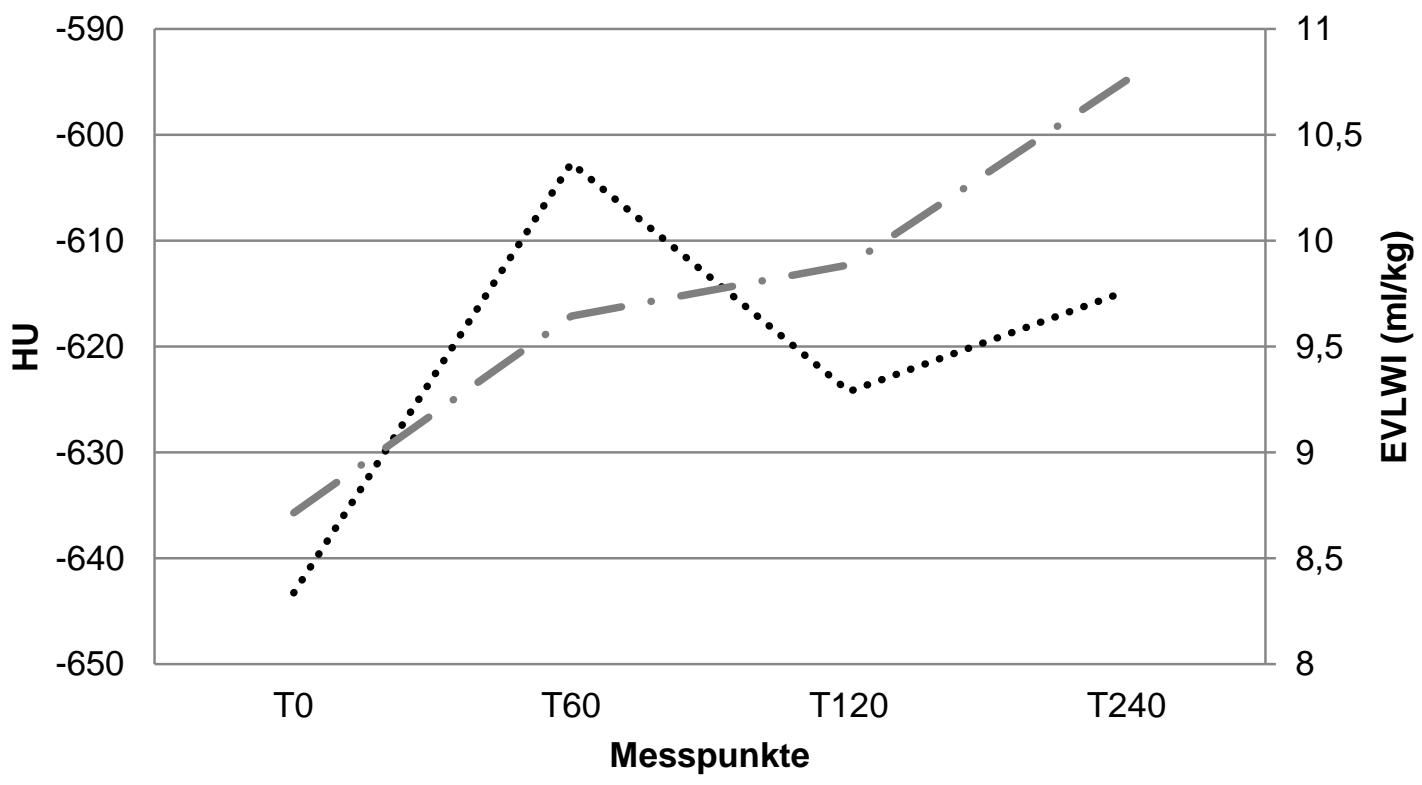

$\mathrm{HU}-\cdot \mathrm{EVLWI}$

Innerhalb der AICH-Gruppe stieg mit dem EVLWI auch die Lungenparenchymdichte an. 
Abbildung 44: Entwicklung HU vs. EVLWI - ARDS - $T_{0}-T_{240}$

HU vs. EVLWI - ARDS Gruppe

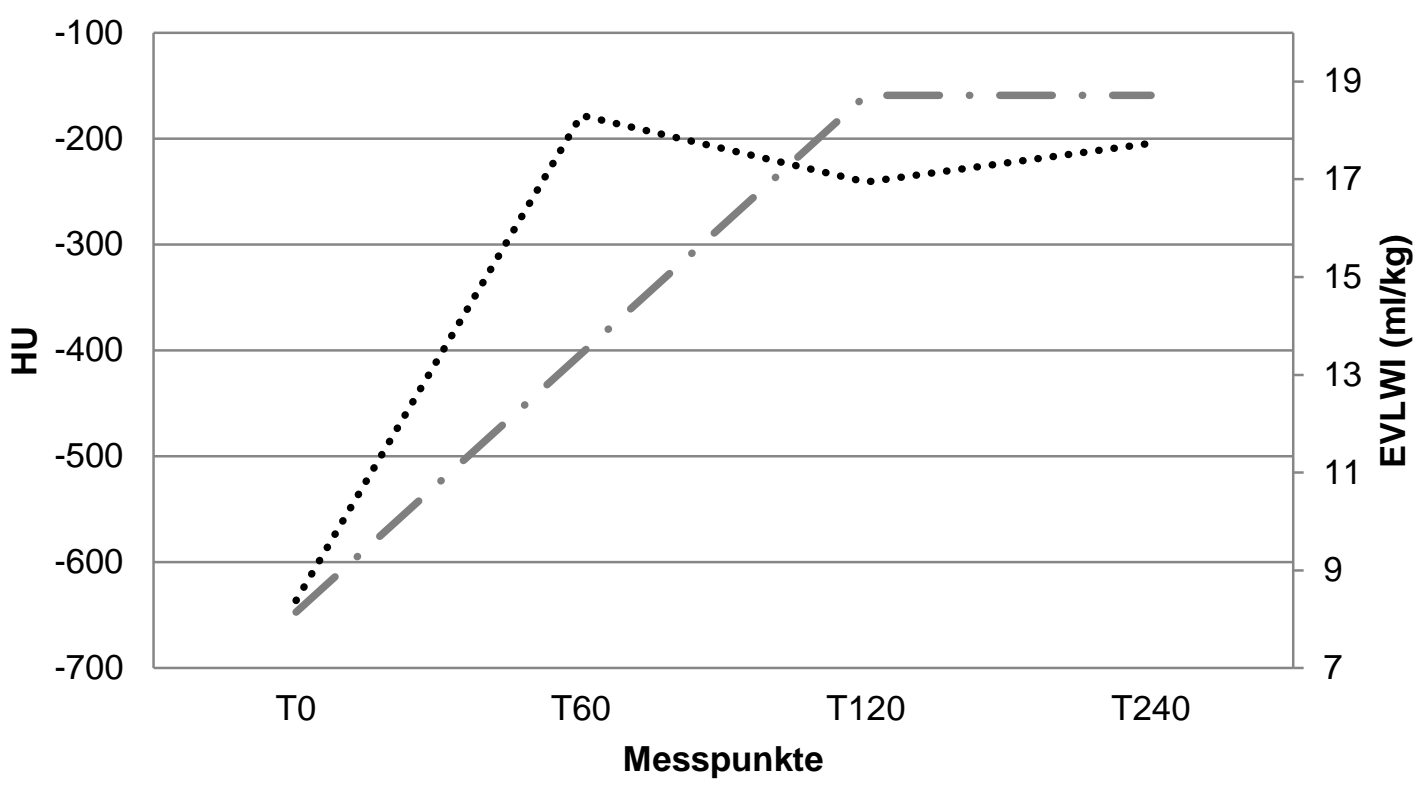

$\mathrm{HU}-\cdot \mathrm{EVLWI}$

Innerhalb der ARDS-Gruppe war bei einem deutlichen Anstieg des EVLWI auch eine deutliche Zunahme der Lungenparenchymdichte zu beobachten.

Abbildung 45: Entwicklung HU vs. EVLWI - ARDS +AICH - $T_{0}-T_{240}$

HU vs. EVLWI - ARDS + AICH Gruppe

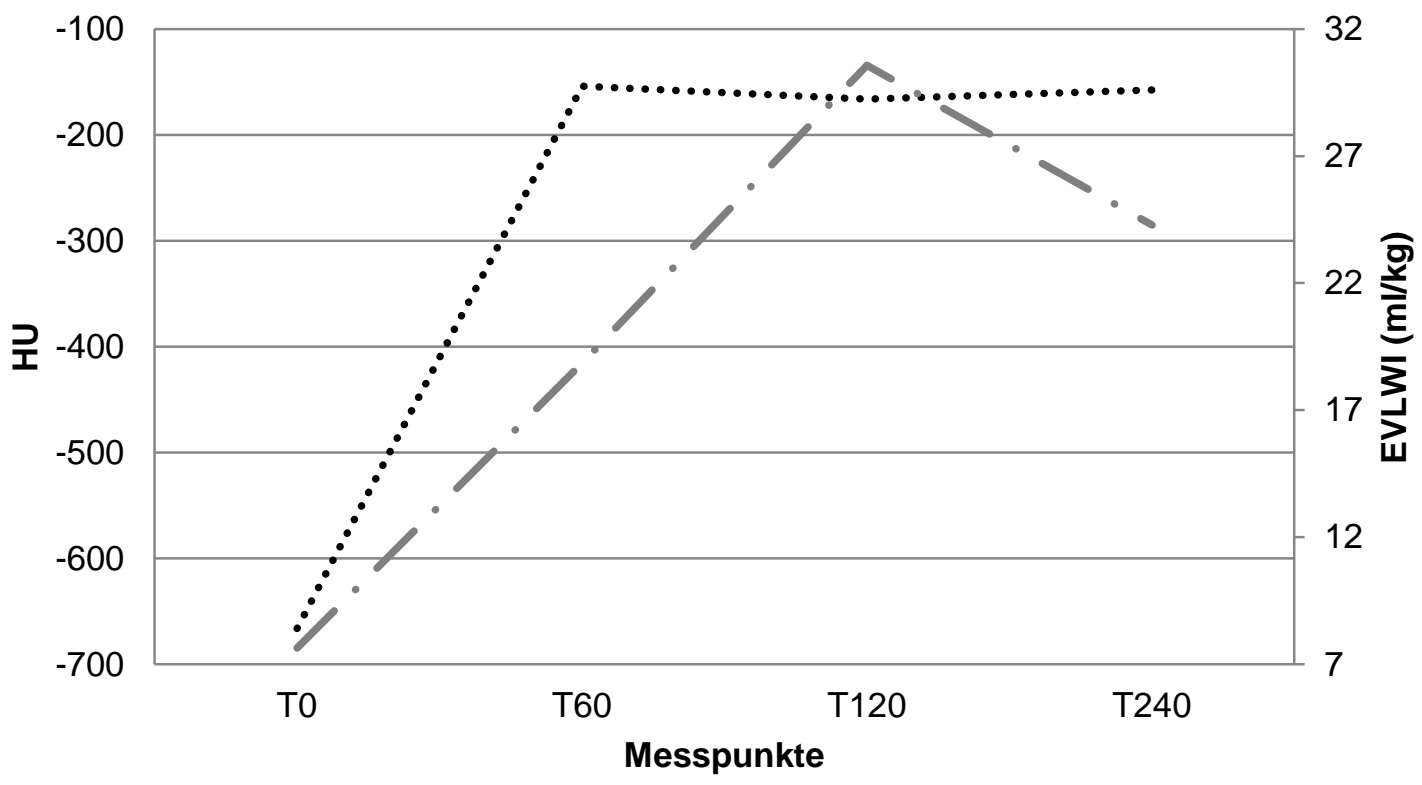

$\mathrm{HU}-\cdot \mathrm{EVLWI}$

Der größte Anstieg von EVLWI fand sich in der Kombinationsgruppe; er ging ebenfalls mit einem deutlichen Anstieg der Lungenparenchymdichte einher. 


\subsection{Histologische Untersuchung der Lunge}

Die Ergebnisse der histologischen Untersuchung der Lunge sind der Tabelle 15 zu entnehmen. Das Ausmaß der Zellschädigung und der Schweregrad des Schadens waren in der ARDS + AICH-Gruppe signifikant höher $(p<0,05)$ als in den anderen drei Gruppen. In den Gruppen ARDS und ARDS + AICH gab es mehr ödematöse Veränderungen als in den Gruppen Control und $\mathrm{AICH}$. Die meisten ödematösen Veränderungen fanden sich in der ARDS + AICH-Gruppe. Zusätzlich waren Schweinelungen, die durch Ölsäure geschädigt worden waren, deutlich häufiger von pulmonaler Embolisation betroffen.

Tabelle 16: Histopathologisches Scoring der Lungenschädigung

\begin{tabular}{|c|c|c|c|c|c|c|c|c|c|c|c|c|c|c|c|c|c|c|c|c|}
\hline \multicolumn{4}{|c|}{ Control } & \multicolumn{6}{|c|}{$\mathrm{AlCH}$} & \multicolumn{4}{|c|}{ ARDS } & \multicolumn{7}{|c|}{ ARDS + AICH } \\
\hline Lungenscore & 0 & 1 & 2 & 3 & 4 & 0 & 1 & 2 & 3 & 4 & 0 & 1 & 2 & 3 & 4 & 0 & 1 & 2 & 3 & 4 \\
\hline $\begin{array}{l}\text { Ausmaß } \\
0-4\end{array}$ & 99 & 5 & 1 & 0 & 0 & 98 & 7 & 0 & 0 & 0 & 0 & 27 & 57 & 20 & $1^{\circ}$ & 0 & 10 & 49 & 36 & $10^{\circ 1}$ \\
\hline $\begin{array}{l}\text { Schweregrad } \\
0-3\end{array}$ & 98 & 7 & 0 & 0 & - & 93 & 12 & 0 & 0 & - & 0 & 18 & 65 & $22^{\circ}$ & - & 0 & 4 & 80 & $21^{\circ 1}$ & - \\
\hline & & & & & & & & & & & & & & & & & & & & \\
\hline $\begin{array}{l}\text { elektasen } \\
0 \text { - } 4\end{array}$ & 47 & 39 & 16 & 3 & 0 & 36 & 30 & 28 & 11 & 0 & 61 & 39 & 5 & 0 & $0^{\circ}$ & 49 & 38 & 13 & 5 & $0^{\wedge}$ \\
\hline $\begin{array}{l}\text { Ödem } \\
0 \text { - } 4\end{array}$ & 103 & 2 & 0 & 0 & 0 & 104 & 1 & 0 & 0 & 0 & 47 & 27 & 17 & 12 & $2^{\circ}$ & 31 & 55 & 16 & 3 & $0^{\circ \wedge}$ \\
\hline $\begin{array}{l}\text { Embolisation } \\
0-3\end{array}$ & 97 & 4 & 4 & 0 & - & 90 & 11 & 3 & 1 & - & 66 & 17 & 19 & $3^{\circ}$ & - & 77 & 14 & 11 & $3^{\circ}$ & - \\
\hline
\end{tabular}

Darstellung als Mittelwerte mit Standardabweichung * Signifikanter Unterschied zum Zeitpunkt $T_{0}(p<0,05)$; ${ }^{\circ}$ Signifikanter Unterschied im Vergleich zur Kontrollgruppe $(p<0,05): \wedge$ Signifikanter Unterschied zur ARDS-Gruppe $(p<0,05)$ (Publiziert in Intensive Care Med (2011) 37:1182-1191, Electronic supplementary material (ESM), Table. 4) 


\section{Diskussion}

\subsection{Einleitung}

In der vorliegenden Arbeit wurden die Wechselwirkungen einer akuten Hirndrucksteigerung ( $\mathrm{AICH}$, Druckmodell, Ballonkatheter) sowie einer akuten Lungenschädigung (Ölsäuremodell) am Tiermodell (Hausschwein) untersucht. Im Vordergrund standen hierbei die computertomographische Dichtemessung des Lungenparenchyms sowie die Veränderungen des extravaskulären Lungenwassers (EVLW), gemessen mit Hilfe der Thermodilutionsmethode (PICCO-Monitoring) über einen Zeitraum von 240 min.

Die Versuchsreihe konnte zeigen, dass eine akute Erhöhung des intrakraniellen Drucks (ICP > $30 \mathrm{mmHg}$ ) in gesunden wie auch in bereits vorgeschädigten Schweinelungen eine Erhöhung des extravaskulären Lungenwassers und eine Zunahme der pulmonalen Dichte, gemessen in Hounsfield-Einheiten, bewirken kann, ohne zwangsläufig den Gasaustausch oder die Hämodynamik klinisch relevant zu verändern. Darüber hinaus zeigen die Untersuchungen, dass eine akute intrakranielle Druckerhöhung bei Schweinen mit bereits bestehendem Lungenschaden zu einer Potenzierung der Lungenschädigung führen kann. Eine retrospektive Studie von Rogers et al. (Rogers et al. 1995) untersuchte den möglichen Einfluss eines Schädel-Hirn-Traumas auf die Lunge. Sie zeigte, dass Lungen von Patienten, die eine Verletzung des zentralen Nervensystems erlitten hatten, postmortal ein höheres Gewicht aufwiesen als Lungen von Patienten mit anderen, nicht neurologischen Verletzungen. Rogers et al. führten dies auf ein pulmonales Ödem, dessen Entwicklung unmittelbar mit dem Trauma einsetzte, zurück. Mascia et al. (Mascia et al. 2008) untersuchten einen ähnlichen Zusammenhang und zeigten mit ihren Ergebnissen, dass Patienten mit neurologischen Diagnosen wie z.B. einem Schädel-Hirn-Trauma oder einem zerebrovaskulären Ereignis trotz eines geringeren Patientenalters und reduzierten Komorbidiäten häufiger ein Acute Respiratory Distress Syndrome (ARDS) entwickelten. Der negative Einfluss einer intrakraniellen Verletzung unterschiedlicher Genese auf die Lunge ist seit längerem bekannt; er verschlechtert das neurologische Outcome der Patienten zusätzlich. Eine Studie an 137 Traumapatienten aus Kalifornien (Holland et al. 2003) beschrieb, dass Patienten, die sowohl eine Verletzung des Gehirns als auch eine Schädigung der Lunge erlitten hatten, nicht nur eine verlängerte Beatmungszeit und ein schlechteres neurologisches Outcome, sondern auch eine um 18\% erhöhte Mortalität aufwiesen. 
Die pathogenetischen Mechanismen, über, die eine isolierte Schädigung des Gehirns zu Veränderungen der Lungendichte und Erhöhung des extravaskulären Lungenwassers führen könnte, werden kontrovers diskutiert. Der genaue Mechanismus ist aufgrund der komplexen pathophysiologischen Zusammenhänge bis heute nicht restlos geklärt. Ein geläufiger Begriff, der versucht, die pathophysiologischen Veränderungen zu beschreiben, ist das neurogene pulmonale Ödem (NPE = Neurogenic Pulmonary Edema) (Simon 1993). Hierbei handelt es sich um ein pulmonales Ödem, welches in direktem zeitlichen Zusammenhang mit einer plötzlichen neurologischen Schädigung steht. Das Vorkommen einer pulmonalen Dysfunktion bzw. eines NPE nach SchädelHirn-Trauma ist aufgrund zahlreicher Untersuchungen mittlerweile anerkannt (Baumann et al. 2007, Fontes et al. 2003, Rogers et al. 1995). Definiert wird es durch eine Erhöhung des extravaskulären Lungenwassers und eine Beeinträchtigung des pulmonalen Gasaustauschs nach einem akuten neurologischen Schaden (Mascia 2009, Touho et al. 1989). Man geht seither davon aus, dass ein neurogenes pulmonales Ödem auf zwei Hauptursachen beruht: zum einem auf einem erhöhten intravaskulären Druck, verursacht durch eine zentrale Sympathikusaktivierung (McClellan et al. 1989, Smith und Matthay 1997, Theodore und Robin 1976), und zum anderen durch eine Permeabilitätserhöhung pulmonaler Kapillaren, bedingt durch eine inflammatorische Reaktion (Hutchinson et al. 2007, McKeating und Andrews 1998, Ott et al. 1994). Welcher der beiden Mechanismen hauptverantwortlich für das pulmonale Ödem ist, konnte bisher nicht eindeutig geklärt werden. Mascia et al. gehen in ihrer Arbeit davon aus, dass sowohl eine massive Sympathikusaktivierung als auch eine inflammatorische Reaktion im Sinne eines „Double Hit“-Modells zur Entstehung eines neurogenen pulmonalen Ödems beitragen (Mascia 2009).

\subsection{Hämodynamik}

\subsubsection{Blutdruck, Herzfrequenz, Schlagvolumen, Herzzeitvolumen}

Ein erklärender pathophysiologischer Mechanismus besteht in einer zentral verursachten Sympathikusaktivierung, die das beobachtete neurogene pulmonale Ödem in unserer Untersuchung ausgelöst haben könnte. Pathophysiologisch kommt es nach Theodore et al. durch eine zentral vermittelte, massive Sympathikusaktivierung zu einer starken, generalisierten, jedoch möglicherweise nur vorübergehenden Vasokonstriktion (Theodore und Robin 1976). Diese Vasokonstriktion bedingt eine Umverteilung von Blutvolumen aus den Widerstandsgefäßen des systemischen Kreislaufs in das Nieder- 
drucksystem der pulmonalen Gefäßstrombahn. Folglich würde es zu einem Anstieg des pulmonalvaskulären Drucks mit konsekutiver Erhöhung des hydrostatischen Drucks im Gefäßlumen sowie des intrapulmonalen Blutvolumens kommen (Theodore und Robin 1976). Die Konsequenz wäre eine Schädigung der pulmonalen Kapillaren und das Übertreten von Blutbestandteilen (Zellen, Protein, Flüssigkeit) in das Interstitium und in die Alveolen. Gestützt wird diese Überlegung von Smith et al., die in einer klinischen Studie an 12 Patienten mit traumatischem Hirnschaden das Verhältnis von Plasmaproteinen im alveolären Ödem zum Blut untersuchten (Smith und Matthay 1997) und so Rückschlüsse auf einen hydrostatischen Ursprung der Proteine ziehen konnte. Betrachtet man nun die Ergebnisse der hämodynamischen Messwerte der Versuchstiere, so fällt auf, dass sich zwar der mittlere pulmonalarterielle Druck in den Gruppen ARDS und ARDS + AICH im Vergleich zur Control erhöhte, nicht jedoch der mittlere arterielle Druck. Ähnlich verhält es sich mit der AICH-Gruppe, in der sogar die mittleren pulmonalarteriellen Drücke im Vergleich zur Kontrollgruppe konstant blieben. Dies legt die Vermutung nahe, dass hinsichtlich des gesteigerten hydrostatischen Drucks im pulmonalen Gefäßbett der Einfluss einer erhöhten Sympathikusaktivität auf die Entstehung des neurogenen pulmonalen Ödems möglicherweise geringer sein könnte als bisher angenommen. Eine Limitierung stellt jedoch die Tatsache dar, dass das in der vorliegenden Untersuchung angewandte Ölsäuremodell per se zu einer Erhöhung des mittleren pulmonalarteriellen Drucks führt. Bei stets gleicher Ölsäureapplikation in den Gruppen ARDS und ARDS + AICH würde man dann jedoch aufgrund der intrakraniellen Druckerhöhung einen noch größeren Anstieg der pulmonalarteriellen Drücke, bedingt durch eine Sympathikusaktivierung, erwarten. Des Weiteren wurden die hämodynamischen Parameter nur zu den einzelnen Messpunkten und nicht unmittelbar nach der akuten ICP-Erhöhung bestimmt, so dass eine nicht detektierte Sympathikuserhöhung denkbar erscheint. Zusätzlich wäre vielleicht eine plasmatische Katecholaminmessung in der Lage gewesen, den Sympathikotonus genauer zu erfassen. Anhalt für eine verstärkte Sympathikusaktivität im untersuchten Zeitraum war die signifikante Zunahme des Herzzeitvolumens in der Gruppe AICH. In den Gruppen ARDS und ARDS + AICH fand sich ein signifikanter Anstieg der Herzfrequenz zu den Messpunkten $T_{60}, T_{120}$ und $T_{240}$. Hierbei ist jedoch zu beachten, dass das Schlagvolumen bei allen Tieren der Gruppen ARDS und ARDS + AICH zu den Zeitpunkten $T_{60}, T_{120}$ und $T_{240}$ im Vergleich zum Messpunkt $T_{0}$ signifikant abnahm und das Herzzeitvolumen der Gruppen ARDS und ARDS + AICH somit annähernd konstant blieb. Eine reine Zunahme der Herzfre- 
quenz bei gleichzeitiger Abnahme des Schlagvolumens scheint als pathophysiologischer Erklärungsversuch für ein neurogenes pulmonales Ödem auf dem Boden einer massiven Sympathikusaktivierung fraglich. Zu einem ähnlichen Ergebnis kamen McClellan et al. in einer Studie an Hunden, in der sie den Einfluss hämodynamischer Veränderungen auf die pulmonale Gefäßpermeabilität hinsichtlich einer Proteinextravasation untersuchten. Es konnte gezeigt werden, dass unabhängig von hämodynamischen Veränderungen ein weiterer Einfluss seitens des Nervensystems auf die pulmonalvaskuläre Permeabilität bestehen muss, der die Parenchymveränderung bewirkt (McClellan et al. 1989). Zu einem anderen Ergebnis kamen Contant et al. in einer klinischen Studie, die sich mit der Therapie eines intrakraniellen Hochdrucks und ARDSEntwicklung beschäftigte. Richtet man die Therapie nach der Aufrechterhaltung eines ausreichenden cerebralen Blutflusses mit hohen systemischen arteriellen Drücken aus, so erhöht sich das Risiko für die Entwicklung eines ARDS (Contant et al. 2001).

\subsubsection{Intrathorakales Blutvolumen}

Der Einfluss eines erhöhten intrathorakalen Blutvolumens auf das neurogene pulmonale Ödem, im Sinne eines Shifts von Volumen aus dem Hochdruck- in das Niederdrucksystem, wie er von Theodore postuliert wird (Theodore und Robin 1976), lässt sich durch unsere Untersuchungen nicht nachvollziehen. In keiner der vier Versuchsgruppen fand sich zu den definierten Messpunkten eine signifikante Veränderung des intrathorakalen Blutvolumens, obwohl pulmonale Veränderungen sowohl in der Computertomographie als auch beim extravaskulären Lungenwasser offensichtlich waren.

\subsection{Gasaustausch}

Der pulmonale Gasaustausch wurde in dieser Arbeit mit Hilfe von arteriellen Blutgasanalysen und pulsoxymetrisch gemessener Sauerstoffsättigung überwacht. Der arterielle Sauerstoffpartialdruck $\left(\mathrm{PaO}_{2}\right)$ wie auch der arterielle Kohlendioxidpartialdruck $\left(\mathrm{PaCO}_{2}\right)$ waren sowohl in der Gruppe Control als auch in der Gruppe AICH über alle Messpunkte konstant. Verglichen mit der Kontrollgruppe kommt es also im untersuchten Zeitraum zu keiner signifikanten Beeinträchtigung des Gasaustauschs in der Gruppe $\mathrm{AICH}$. In den Gruppen ARDS und ARDS + $\mathrm{AICH}$ nahm der $\mathrm{PaO}_{2}$ jeweils nach Induktion der Lungenschädigung ab, so dass zu jedem Zeitpunkt die ARDS-Kriterien $\left(\mathrm{PaO}_{2}<200\right.$ mmHg bei $\mathrm{FiO}_{2} 1,0$ ) in beiden Gruppen erfüllt waren. Der $\mathrm{PaCO}_{2}$ stieg in diesen beiden Gruppen nach Schädigung der Lunge durch Ölsäure signifikant an. Es fanden sich jedoch keinerlei signifikante Unterschiede von $\mathrm{PaO}_{2}$ und $\mathrm{PaCO}_{2}$ im direkten Vergleich der 
ARDS und ARDS + AICH-Gruppe. Arterielle Blutgasanalysen allein sind somit im vorliegenden Modell weder im Rahmen eines isolierten Schädel-Hirn-Traumas noch in Kombinationsschädigungen geeignet, alle Veränderungen der Lungenfunktion in einem Zeitraum von 240 min quantifizierbar zu machen. Sie können lediglich als Hilfsmittel zur Beurteilung z.B. des aktuellen Beatmungsregimes bei bereits bestehendem ARDS herangezogen werden, um eventuell die Therapie anpassen zu können. Es sei ausdrücklich darauf hingewiesen, dass Veränderungen der Blutgase bei einer Verlängerung des Beobachtungszeitraums nicht ausgeschlossen werden können. Andere diagnostische Hilfsmittel sind nötig, die eine frühzeitige Erkennung und zeitgerechte Therapie eines pulmonalen Ödems nach neurologischem Trauma gestatten.

\subsection{CT-Analyse der Lunge}

Der zweite mögliche Mechanismus für ein neurogenes pulmonales Ödem beruht auf der Überlegung, dass das Cerebrum selbst Mediatoren in die Blutbahn ausschüttet, die dort wiederum eine systemische, inflammatorische Reaktion an verschiedenen Strukturen hervorrufen können, insbesondere an der Lunge. Das Cerebrum wurde lange Zeit als „immunologisch privilegiertes" Organ betrachtet, das weder für immunologische Prozesse verantwortlich gemacht werden kann, noch von systemischen Immunreaktionen beeinflusst wird. Lucas et al. konnten jedoch zeigen, dass das Cerebrum zahlreiche inflammatorisch wirksame Mediatoren (Zytokine, Prostaglandine, freie Radikale), die systemisch wirken, bereitstellen kann (Lucas et al. 2006). Dem eher mechanischen Modell der massiven Sympathikusaktivierung wurde nun die Überlegung entgegengestellt, dass es nach einem akuten Hirnschaden, gleich welcher Ätiologie, zu einer intracerebralen Produktion pro-inflammatorischer Zytokine kommt, die in den systemischen Kreislauf gelangen und dort zu einer Entzündungsreaktion führen.

Die anfängliche Antwort der Lunge auf eine intrakranielle Schädigung lässt sich mittels einer konventionellen Röntgenaufnahme des Thorax (Halperin et al. 1985) oder unmittelbarer arterieller Blutgasanalysen nicht nachweisen. Voraussetzung hierfür wäre ein deutlich erkennbares pulmonales Ödem mit Einschränkung der Diffusionskapazität für Sauerstoff und damit verbundener Erhöhung flüssiger Bestandteile im Lungengewebe, welche dann im Thorax-Röntgenbild sichtbar wären. Quantitative Analysen von computertomographischen Bildern der Lunge wurden bereits als frühe Indikatoren sowie möglicherweise prognostisch und therapeutisch relevante Hilfsmittel gehandelt, um diese frühen Veränderungen zu detektieren. So versuchten Gattinoni et al. 2006 mit Hilfe 
quantitativer Computertomographie, eine Vorhersage über potentiell rekrutierbare Lungenareale bei ARDS-Patienten zu treffen (Gattinoni et al. 2006). Sie waren dadurch in der Lage, zwischen Patienten mit verschiedenen Rekrutierungspotentialen in Abhängigkeit des PEEP zu unterscheiden.

\subsubsection{Analyse der Lungendichte}

Im Folgenden sollen nun die Ergebnisse der mittleren Lungendichte, ausgedrückt in Hounsfield-Einheiten, der Gasgehalt und die prozentuale Verteilung von normal, wenig und nicht belüftetem Lungengewebe der verschiedenen Versuchsgruppen für die gesamte Lunge diskutiert werden.

In der Kontrollgruppe nahm die Dichte des Lungengewebes vom Zeitpunkt $T_{0}$ zum Zeitpunkt $T_{240}$ im Mittel um $38 \mathrm{HU}( \pm 23)$ ab. Es kam also zu einer erhöhten Strahlendurchlässigkeit. Diese Abnahme lässt sich am ehesten auf ein Recruitment zurückführen, das in den übrigen Gruppen nicht beobachtet werden konnte. Die Parenchymdichte in der Gruppe AICH nahm über 240 Minuten um $28 \mathrm{HU}( \pm 25)$ zu. Trotz anfänglich $\left(T_{0}\right)$ höherer Dichtewerte in der Gruppe AICH, verglichen mit der Kontrollgruppe, kam es nicht zu einem Recruitment (siehe Abbildung 29), sondern zu einer verminderten Strahlendurchlässigkeit. Ein signifikanter Unterschied $(p<0,05)$ zwischen der Kontrollgruppe und der AlCH-Gruppe konnte zu den Zeitpunkten $T_{0}, T_{60}, T_{120}$ und $T_{240}$ festgestellt werden.

In der Gruppe ARDS nahm die Dichte um $431 \mathrm{HU}( \pm 80)$ und in der Gruppe AICH + ARDS um $509 \mathrm{HU}( \pm 59)$ zu (siehe Abbildung 30). Beide Gruppen erhielten stets eine konstante, gewichtsadaptierte Lungenschädigung durch Ölsäure. Die Hounsfield Units betreffend, konnten zwischen den Gruppen ARDS und AICH + ARDS signifikante $(p<$ $0,05)$ Unterschiede zu den Zeitpunkten $T_{120}$ und $T_{240}$ gemessen werden.

Es kam also sowohl im Vergleich der Gruppen Control und AICH als auch der Gruppen ARDS und ARDS + AICH jeweils bei Tieren mit einer Hirndrucksteigerung zu einer Erhöhung der Lungenparenchymdichte, gemessen in Hounsfield Units.

Diese Dichtezunahmen gingen stets mit einer Abnahme des Gasgehaltes ( $\mathrm{ml}$ Gas/ Gramm Gewebe) einher. Die Abnahme des Gasgehaltes war zwischen den Gruppen Control und $\mathrm{AICH}$ zu den Zeitpunkten $\mathrm{T}_{60}, \mathrm{~T}_{120}$ und $\mathrm{T}_{240}$ signifikant $(\mathrm{p}<0,05)$ und zwischen der ARDS und ARDS + AICH-Gruppe zu allen Messpunkten.

Außerdem konnte eine signifikante Abnahme der normal ventilierten Lungenareale in der Gruppe AICH im Vergleich zur Kontrollgruppe zu den Zeitpunkten $T_{0}, T_{60}, T_{120}$ und $T_{240}$ und eine Zunahme der schlecht und nicht ventilierten Bereiche zu den Zeitpunkten $T_{0}, T_{60}, T_{120}$ und $T_{240}$ festgestellt werden. In der Gruppe ARDS + AICH nahm der Anteil 
normal ventilierter Lungenareale zu den Zeitpunkten $T_{60}$ und $T_{240}$ im Vergleich zur ARDS-Gruppe signifikant $(p<0,05)$ ab und der Anteil nicht ventilierter Bereiche im Vergleich zur ARDS-Gruppe zum Zeitpunkt $\mathrm{T}_{120} \mathrm{zu}$.

Die Versuchstiere zeigten also sowohl im Vergleich der Gruppen Control vs. AICH als auch der Gruppen ARDS vs. ARDS + AICH einen deutlichen Anstieg der Lungendichte, gemessen in Hounsfield Units, und eine Veränderung der alveolären Ventilation hin zu Atelektasenbildung. Die statistisch signifikanten Unterschiede der mittleren Lungendichte zum Zeitpunkt $T_{240}$ Minuten haben initial sicherlich keine klinische Bedeutung. Sie könnten sich jedoch im Verlauf aggravieren, so dass es zu einer relevanten Oxygenierungsstörung kommt, und könnten somit als Anhaltspunkte und zur Verlaufsbeobachtung über einen längeren Zeitraum dienen.

Ähnliche Ergebnisse zeigte die Analyse der regionalen Verteilung von Parenchymdichte, Gasgehalt sowie normal, schlecht und nicht belüfteten Lungenarealen. Die mittlere Lungendichte unterschied sich signifikant $(p<0,05)$ zwischen den Gruppen Control und AICH in den Segmenten 1,3,5,6,9,10 zum Zeitpunkt $T_{60}$ und in den Segmenten 3,9,10 zum Zeitpunkt $\mathrm{T}_{240}$.

Im direkten Vergleich der Gruppen ARDS und AICH + ARDS konnte eine signifikante $(p<0,05)$ Zunahme der mittleren Lungendichte in den Segmenten 9 und $10 \mathrm{zu}$ den Zeitpunkten $T_{60}$ und $T_{120}$ beobachtet werden. Die Abbildungen 39 und 40 zeigen den Unterschied $\left(T_{240}-T_{0}\right)$ der mittleren Lungendichte in Hounsfield Units zwischen der Kontroll- und der AICH-Gruppe sowie zwischen der ARDS und ARDS + AICH-Gruppe von nicht-abhängigen zu abhängigen Lungenarealen.

Betrachtet man die delta $\mathrm{HU}\left(\mathrm{T}_{240}-\mathrm{T}_{0}\right)$, lässt sich feststellen, dass es in allen Segmenten der Kontrollgruppe zu einer Abnahme und in der AICH-Gruppe zu einer Zunahme der Parenchymdichte kam (Abbildung 39).

Die delta $H U\left(T_{240}-T_{0}\right)$ in allen Segmenten der ARDS-Gruppe und sogar noch stärker in der ARDS + AICH-Gruppe nahmen ebenfalls zu. Es handelte sich meist um eine schwerkraftabhängige Veränderung, die im Bereich der abhängigeren Lungenpartien stärker ausgeprägt war. Ähnliche Veränderungen bezüglich der regionalen Verteilung eines ARDS wurden bereits in klinischen Studien nachgewiesen (Pelosi et al. 1994). Die bisherigen Ergebnisse sprechen für die Überlegung, dass neben einer möglichen Sympathikusaktivierung vor allem zentral ausgeschüttete Mediatoren eine Permeabilitätsveränderung des Lungenparenchyms bewirken können. Barklin et al. wiesen erst 
kürzlich nach, dass es bei hirntoten Schweinen zu signifikanten Veränderungen der intrapulmonalen Flüssigkeit und Proteinkonzentrationen kommt (Barklin et al. 2009).

Der Gasgehalt ( $\mathrm{ml}$ Gas/g Gewebe) war im Vergleich signifikant $(p<0,05)$ höher in der Kontroll-Gruppe verglichen mit der AICH-Gruppe in den Segmenten 1,4,7,8,9 zum Zeitpunkt $T_{60}$ und in den Segmenten 9 und 10 zum Zeitpunkt $T_{240}$. Der prozentuale Anteil nicht und schlecht belüfteter Lungenareale zum Zeitpunkt $T_{60}$ war signifikant $(p<0,05)$ höher in den Segmenten 1-9 und zum Zeitpunkt $T_{240}$ in den Segmenten 2,3,5,6,7,8 in der Gruppe AICH verglichen mit der Kontroll-Gruppe. Außerdem fand sich ein signifikanter $(p<0,05)$ Unterschied im prozentualen Anteil normal ventilierter Lungenareale in den Segmenten 3-8 zum Zeitpunkt $T_{120}$. In der Gruppe AICH + ARDS stieg der Anteil nicht und schlecht belüfteter Lungenareale im Segment 10 zum Zeitpunkt $T_{60}$, in den Segmenten 9 und 10 zum Zeitpunkt $T_{120}$ und im Segment 10 zum Zeitpunkt $T_{240}$ im Vergleich zur ARDS-Gruppe signifikant $(p<0,05)$ an. Die Abbildungen 19 und 20 zeigen die prozentuale Veränderung von normal, schlecht und nicht ventilierten Lungenarealen zu den Zeitpunkten $T_{0}-T_{240}$ von nicht-abhängigen zu abhängigen Lungenarealen. In der Gruppe AICH sank der prozentuale Anteil normal ventilierter Lungenareale im Vergleich zur Kontrollgruppe, während der Anteil schlecht ventilierter Areale vorzugsweise in den abhängigen Arealen anstieg. Der Anteil nicht belüfteten Gewebes stieg vor allem in der AICH + ARDS-Gruppe in den abhängigen Lungenarealen verglichen mit der ARDSGruppe.

Akuter Hirndruck allein kann offensichtlich die Menge an schlecht belüfteten Lungenarealen in vormals gesunden Schweinelungen erhöhen; darüber hinaus führt akuter Hirndruck in bereits vorgeschädigten Lungen (ARDS induziert durch Ölsäure) vornehmlich in den abhängigen Bereichen zu einer weiteren signifikanten Zunahme sowohl schlecht als auch nicht belüfteter Lungenareale.

Diese Ergebnisse lassen darauf schließen, dass das hier angewandte Hirndruckmodell zwar zu keiner wesentlichen Veränderung der Hämodynamik an den Messpunkten oder zu einer signifikanten Zunahme des intrathorakalen Blutvolumens führt, aber dennoch signifikante Veränderungen der Lungendichte in vormals gesunden wie auch in kranken Lungen auslöst. Trotz eines nicht signifikant veränderten Herzzeitvolumens (mit Ausnahme der AICH-Gruppe), mittleren arteriellen Blutdrucks, mittleren pulmonalarteriellen Blutdrucks und intrathorakalen Blutvolumens der Gruppen Control und $\mathrm{AICH}$ kommt es über einen Zeitraum von 240 min. zu einer signifikante Veränderung der Lungendichte. Dasselbe gilt für den Vergleich der Gruppen ARDS und ARDS + $\mathrm{AICH}$, wobei es bei 
den Tieren mit Kombinationsschädigung zu einem signifikanten Abfall des Schlagvolumens kam. Avlonitis et al. veröffentlichten eine Arbeit zum Thema Hirntod und potentielle Lungenspender, in der ein Zusammenhang zwischen zerebraler Schädigung und Versagen von transplantierten Lungen besteht (Avlonitis et al. 2003). Sie führten dies auf eine bereits im Spender stattfindende Schädigung des Lungenparenchyms zurück. Die Ergebnisse dieser Arbeit stützen die These, dass es durch eine zerebrale Schädigung, bedingt durch eine ICP-Erhöhung, zu einer Alteration von Lungengewebe kommt. López et al. untersuchten ebenfalls an einem Hirndruckmodell den möglichen Einfluss einer intrakraniellen Drucksteigerung auf die Lunge. Sie kamen zu dem Ergebnis, dass eine Steigerung des Hirndrucks die Lunge anfälliger für ödematöse Veränderungen macht, und unterstreichen somit unsere Ergebnisse (Lopez-Aguilar et al. 2005).

\subsection{Extravaskuläres Lungenwasser}

Zusätzlich zu den Veränderungen der pulmonalen Dichtemessung und des Gasgehaltes kam es durch die intrakranielle Druckerhöhung zu einer Veränderung des extravaskulären Lungenwassers im untersuchten Zeitraum. In der AICH-Gruppe hat sich während des Versuchsablaufes das extravaskuläre Lungenwasser im Gegensatz zur Kontroll-Gruppe signifikant erhöht $(p<0,05)$. Erwartungsgemäß kam es auch in den Gruppen ARDS und ARDS + AICH zu einem signifikanten EVLWI-Anstieg; die größte Veränderung trat jedoch bei den Kombinationstieren zum Zeitpunkt $T_{120}(p<0,05)$ auf. Der Anstieg des extravaskulären Lungenwassers ging stets mit einer Zunahme der Lungendichte in der Computertomographie einher, was die Validität der Daten unterstreicht. Das extravaskuläre Lungenwasser sowie eine quantitative CT-Analyse des Lungengewebes werden als mögliche frühe Marker und prognostisch verwertbare Untersuchungsmethoden bei der Erkennung und Behandlung des ARDS (Gattinoni et al. 2006, Sakka et al. 2002) angesehen. Baumann et al. haben in einer Arbeit über das neurogene pulmonale Ödem hervorgehoben, dass das Krankheitsbild zurzeit unterdiagnostiziert scheint, und weisen daraufhin, dass sich die Mortalität bei Patienten mit Schädel-HirnTrauma und sekundärer Entwicklung eines neurogenen pulmonalen Ödems erhöht. Eine frühzeitige Erkennung des Krankheitsbildes und die schnelle Einleitung einer Therapie sei jedoch erfolgversprechend (Baumann et al. 2007). Die frühzeitige Erkennung eines neurogenen pulmonalen Ödems ist also entscheidend. Sakka et al. untersuchten retrospektiv 373 Patienten bezüglich des gemessenen extravaskulären Lungenwassers und des daraus resultierenden medizinischen Outcomes. Sie stellten fest, dass die Be- 
stimmung des extravaskulären Lungenwassers besser als z.B. Gasaustauschparameter oder radiologische Untersuchungen mit der pulmonalen Funktion und dem Überleben korreliert (Sakka et al. 2002). Zu ähnlichen Ergebnissen kamen Davey-Quinn et al. Zwar hatten überlebende Patienten in dieser klinischen Studie anfänglich höhere Werte für extravaskuläres Lungenwasser, zeigten jedoch gleichzeitig eine vermehrte Abnahme des EVLWI unter der ARDS-Therapie (Davey-Quinn et al. 1999). Auch unsere Ergebnisse sprechen für ein schnelles, signifikantes Ansprechen des extravaskulären Lungenwassers. So fanden sich zwischen den Gruppen Control und $\mathrm{AICH}$ bereits nach 120 Minuten signifikante Unterschiede. Bei den Gruppen ARDS und ARDS + AICH war sogar nach 60 Minuten schon ein signifikanter Unterschied messbar. Abbildung 41 veranschaulicht noch einmal den deutlichen Anstieg. Das extravaskuläre Lungenwasser vermag also im vorliegenden Modell frühzeitig pulmonale Veränderungen nach intrakranieller Druckerhöhung aufzuzeigen. Diese Ergebnisse konnten durch die CT-Diagnostik zusätzlich untermauert werden (siehe Abbildungen 42 und 43).

\subsection{Histologische Untersuchung der Lunge}

Das Ausmaß der Zellschädigung sowie deren Schweregrad war in der ARDS + AICHGruppe am größten. Es kam zu vermehrter Ödembildung in den Gruppen ARDS und ARDS + AICH, verglichen mit der Kontroll- und AICH-Gruppe. Die größten ödematösen Veränderungen waren in der ARDS + AICH-Gruppe zu beobachten. Zusätzlich war das Ölsäuremodell mit einer erhöhten Rate pulmonalarterieller Embolien verbunden. López et al. kamen mit ihrem Versuch zu ähnlichen Ergebnissen. Zwar wurde hier nur eine isolierte intrakranielle Druckerhöhung angewandt, es fanden sich jedoch ebenso ödematöse Veränderung und somit eine erhöhte pulmonalvaskuläre Permeabilität.

\subsection{Eine kritische Betrachtung des Versuchsaufbaus}

Die Veränderung der Lungendichte unterliegt verschiedensten Faktoren wie z.B. Schwankungen des Gas- und Gewebevolumens, des Blutvolumens und des Wassergehaltes im Lungenparenchym. In Zusammenspiel mit dem erhöhten extravaskulären Lungenwasser sowie dem prozentualen Abfall der normal und dem Anstieg der wenigund schlecht belüfteten Lungenareale lässt sich die Zunahme der Lungendichte im CT mit einer vermehrten Flüssigkeitszunahme im Lungenparenchym begründen. Die hierbei stärkere Gewichtung der abhängigen Lungenpartien lässt sich möglicherweise durch eine schwerkraftbedingte Umverteilung des Ödems oder den Kollaps zuvor belüfteter, abhängiger Areale erklären, wie man es auch im klinischen Alltag bei ARDS- 
Patienten beobachten kann (Pelosi et al. 1994). Besonders hervorzuheben ist in diesem Zusammenhang, dass bereits ein Untersuchungszeitraum von 240 Minuten ausreichte, um signifikante Veränderungen des extravaskulären Lungenwassers, auch in gesunden Lungen, durch eine Schädigung mittels akuter intrakranieller Drucksteigerung hervorzurufen. Ergebnisse der quantitativen CT-Untersuchungen sowie des extravaskulären Lungenwassers sind offensichtlich frühe Marker einer pulmonalen Veränderung. Beide Verfahren sind jedoch mit Nebenwirkungen bzw. potentiellen Komplikationen behaftet. Das CT stellt eine nicht unerhebliche Belastung durch ionisierende Strahlung dar, und die Bestimmung des EVLWI erfordert die Kanülierung eines arteriellen Gefäßes und das Einbringen eines Katheters. Eine Übersichtsarbeit im New England Journal of Medicine warnt z.B. vor den Konsequenzen heute durchgeführter CT-Untersuchungen und kommt zu dem Ergebnis, dass diese für 1,5 - 2\% aller zukünftigen Krebserkrankungen verantwortlich sein könnten (Brenner und Hall 2007). Die Autoren weisen aber auch darauf hin, dass in vielen Fällen der Nutzen das Risiko überwiege. Es bleibt also die Verantwortung jedes einzelnen Mediziners, diese Risiko-Nutzen-Abwägung für den speziellen Fall immer wieder aufs Neue vorzunehmen. Die arterielle Punktion von Gefäßen birgt ebenfalls Risiken, die jedoch anders als eine sichere Strahlenexposition im CT durch die Erfahrung des Anwenders minimiert werden können. Zu diesen Risiken zählen z.B. Blutungen, Thrombosen, Infektionen oder arteriovenöse Fisteln.

Um die Ergebnisse der Versuchsreihe besser einordnen zu können, muss auf einige Einschränkungen hingewiesen werden. Erstens konnte die mit 240 Minuten begrenzte Versuchszeit möglicherweise nicht alle denkbaren Interaktionen zwischen der Lunge und dem Gehirn aufzeigen. Die technischen Gegebenheiten, besonders die Nutzung des Computertomographen verlangten, die Versuchsdauer auf 240 Minuten zu begrenzen, womit weitere mögliche Auswirkungen zwischen Cerebrum und Lunge außerhalb des Beobachtungszeitraums liegen könnten. Eine weitere Limitierung stellt die Zahl der Versuchstiere dar, die, bedingt durch den Großtierversuch, auf sieben Tiere pro Gruppe begrenzt war. Das gewählte Verfahren zur intrakraniellen Drucksteigerung (AICH) ist sicher nicht für alle Schädigungsmechanismen repräsentativ, so wäre z.B. anstatt der Insufflation eines Ballonkatheters auch eine Schädigung durch intrakranielle Injektion von Schweineblut denkbar gewesen; die Wahrscheinlichkeit, mit diesem Vorgehen einen ausreichenden, vor allem aber identischen Druckanstieg sicherzustellen, erschien indes zu gering. Damit lässt sich aber nicht ausschließen, dass andere AICH-Methoden möglicherweise einen anderen Einfluss auf die beobachtete Schädigung der Lunge ha- 
ben. Darüber hinaus hat die Wahl einer lungenprotektiven Beatmungsstrategie mit niedrigem Tidalvolumen, PEEP und Bauchlagerung (Mascia et al. 2007, Valenza et al. 2005) möglicherweise Interaktionen zwischen der Lunge und dem Gehirn minimiert. 


\section{Zusammenfassung}

Diese Arbeit untersucht die Hypothese eines möglicherweise additiven Effektes bei einer akuten intrakraniellen Drucksteigerung und einem Acute Respiratory Distress Syndrome. Um die Einflüsse und möglichen Interaktionen der beiden Krankheitsbilder erfassen zu können, wurden in einem randomisierten Tierversuch jede Schädigung isoliert und ihre Kombination verglichen. Zur Quantifizierung möglicher Einflüsse auf die Lunge wurden die radiologische Dichtemessung des Lungenparenchyms, die Bestimmung des extravaskulären Lungenwasserindex (EVLWI), ein histologisches ScoringSystem sowie die Aufzeichnung hämodynamischer (Herzfrequenz, Schlagvolumen, Herzzeitvolumen) und Gasaustauschparameter $\left(\mathrm{PaO}_{2}, \mathrm{PaCO}_{2}\right)$ herangezogen.

Durch den Gruppenvergleich lassen sich folgende Schlussfolgerungen ziehen: Zum Zeitpunkt $T_{0}$ konnten keine Unterschiede in den Gruppen bezüglich Herzfrequenz, Schlagvolumen und Herzzeitvolumen festgestellt werden. Bis auf das Herzzeitvolumen zu den Messpunkten $T_{120}$ und $T_{240}$ änderten sich die hämodynamischen Parameter der Gruppen Control und AICH nicht signifikant. Zwar stieg in den Gruppen ARDS und ARDS + AICH die Herzfrequenz deutlich an, durch das gleichzeitig reduzierte Schlagvolumen änderte sich jedoch das Herzzeitvolumen nicht signifikant.

Die Gasaustauschparameter der Gruppen Control und $\mathrm{AICH}$ wiesen unveränderte $\mathrm{PaO}_{2}$ und $\mathrm{PaCO}_{2}$-Werte auf. In den Gruppen ARDS und ARDS + AICH kam es zu einem Anstieg des $\mathrm{PaCO}_{2}$ sowie zu einem Abfall des $\mathrm{PaO}_{2}$, mit der Tendenz eines stärkeren Abfalls in der ARDS + AICH-Gruppe.

Sowohl in gesunden als auch in geschädigten Schweinelungen führte eine zusätzliche Erhöhung des intrakraniellen Drucks zu einem Anstieg der radiologisch bestimmten Lungendichte. Der hieraus errechnete Gasgehalt und der Anteil normal ventilierter Lungenareale wurde durch intrakranielle Druckerhöhung in gesunden und geschädigten Schweinelungen signifikant $(p<0,05)$ reduziert. Auf der anderen Seite kam es durch die Druckerhöhung zu einer signifikanten $(p<0,05)$ Zunahme schlecht bzw. nicht ventilierter Lungenareale. Eine zusätzliche Segmentanalyse der Lungen konnte nachweisen, dass die genannten Veränderungen besonders die abhängigen Lungenareale betrafen.

Der extravaskuläre Lungenwasserindex veränderte sich in der Kontrollgruppe nicht. In allen anderen Gruppen kam es zu einer kontinuierlichen und teils signifikanten $(p<0,05)$ Zunahme des EVLWI.

Die größten histopathologischen Veränderungen im Sinne einer zellulären Schädigung erfolgten in der ARDS + AICH-Gruppe $(p<0,05)$. Tiere mit einer Lungeschädigung zeig- 
ten einen insgesamt größeren Anteil ödematöser Veränderungen und pulmonalvaskulärer Embolisation als Tiere ohne Lungenschädigung.

Zusammenfassend führt auf Grund der vorliegenden Ergebnisse und im gewählten Untersuchungsaufbau eine akute Erhöhung des intrakraniellen Drucks zu einer Schädigung zuvor gesunder Schweinelungen; darüber hinaus aggraviert sie die Schädigung in zuvor bereits pathologisch verändertem Lungengewebe. 


\section{Literaturverzeichnis}

Abel SJ, Finney SJ, Brett SJ, Keogh BF, Morgan CJ, Evans TW (1998): Reduced mortality in association with the acute respiratory distress syndrome (ARDS). Thorax $\underline{53}, 292-294$

Amato MB, Barbas CS, Medeiros DM, Magaldi RB, Schettino GP, Lorenzi-Filho G, Kairalla RA, Deheinzelin D, Munoz C, Oliveira R (1998): Effect of a protective-ventilation strategy on mortality in the acute respiratory distress syndrome. N Engl J Med $\underline{338}, 347-$ 354

Arroliga AC, Ghamra ZW, Perez Trepichio A, Perez Trepichio P, Komara JJ, Smith A, Wiedemann HP (2002): Incidence of ARDS in an adult population of northeast Ohio. Chest $\underline{121}$, 1972-1976

Ashbaugh DG, Bigelow DB, Petty TL, Levine BE (1967): Acute respiratory distress in adults. Lancet 1967, II, 319-323

Atabai K, Matthay MA (2002): The pulmonary physician in critical care. 5: Acute lung injury and the acute respiratory distress syndrome: definitions and epidemiology. Thorax $\underline{57}, 452-458$

Avlonitis VS, Fisher AJ, Kirby JA, Dark JH (2003): Pulmonary transplantation: the role of brain death in donor lung injury. Transplantation $\underline{75}$, 1928-1933

Baker CS, Evans TW, Randle BJ, Haslam PL (1999): Damage to surfactant-specific protein in acute respiratory distress syndrome. Lancet $\underline{353}, 1232-1237$

Barklin A, Theodorsson E, Tyvold SS, Larsson A, Granfeldt A, Sloth E, Tonnesen E (2009): Alteration of neuropeptides in the lung tissue correlates brain death-induced neurogenic edema. J Heart Lung Transplant 28, 725-732

Baudouin SV (2004): Exogenous surfactant replacement in ARDS--one day, someday, or never? N Engl J Med $\underline{351}, 853-855$

Baumann A, Audibert G, McDonnell J, Mertes PM (2007): Neurogenic pulmonary edema. Acta Anaesthesiol Scand $\underline{51}, 447-455$

Becker HF, Jerrentrup A (2001): Beatmungstherapie bei akuter respiratorischer Insuffizienz - gegenwärtiger Stand. Internist (Berl) 느, 342 - 348

Bell RC, Coalson JJ, Smith JD, Johanson WG (1983): Multiple organ system failure and infection in adult respiratory distress syndrome. Ann Intern Med 99, 293-298

Bernard GR, Artigas A, Brigham KL, Carlet J, Falke K, Hudson L, Lamy M, Legall JR, Morris A, Spragg R (1994): The American-European Consensus Conference on ARDS. Definitions, mechanisms, relevant outcomes, and clinical trial coordination. Am J Respir Crit Care Med 149, 818-824 
Biro P, Wiedemann K (1999): Jet ventilation and anaesthesia for diagnostic and therapeutic interventions of the airway. Anaesthesist $\underline{48}, 669-685$

Boussat S, Jacques T, Levy B, Laurent E, Gache A, Capellier G, Neidhardt A (2002): Intravascular volume monitoring and extravascular lung water in septic patients with pulmonary edema. Intensive Care Med 28, 712-718

Bratton SL, Davis RL (1997): Acute lung injury in isolated traumatic brain injury. Neurosurgery $\underline{40}, 707-712$

Brenner DJ, Hall EJ (2007): Computed tomography--an increasing source of radiation exposure. N Engl J Med $\underline{357}$, 2277-2284

Brower RG, Ware LB, Berthiaume Y, Matthay MA (2001): Treatment of ARDS. Chest $\underline{120}, 1347-1367$

Brown AW, Elovic EP, Kothari S, Flanagan SR, Kwasnica C (2008): Congenital and acquired brain injury. 1. Epidemiology, pathophysiology, prognostication, innovative treatments, and prevention. Arch Phys Med Rehabil 89 Suppl 1, 3-8

Brun-Buisson C, Minelli C, Bertolini G, Brazzi L, Pimentel J, Lewandowski K, Bion J, Romand JA, Villar J, Thorsteinsson A (2004): Epidemiology and outcome of acute lung injury in European intensive care units. Results from the ALIVE study. Intensive Care Med $\underline{30}, 51-61$

Carli P, Orliaguet G (2004): Severe traumatic brain injury in children. Lancet $\underline{363}$, 584 585

Coetzee A, Rousseau H, Lahner D (1996): Acute pulmonary hypertension and right ventricular failure in adult respiratory distress syndrome. S Afr Med J 86 Suppl 3, 147151

Contant CF, Valadka AB, Gopinath SP, Hannay HJ, Robertson CS (2001): Adult respiratory distress syndrome: a complication of induced hypertension after severe head injury. J Neurosurg 95, 560-568

D'Ambrosio R, Perucca E (2004): Epilepsy after head injury. Curr Opin Neurol 17, 731735

Davey-Quinn A, Gedney JA, Whiteley SM, Bellamy MC (1999): Extravascular lung water and acute respiratory distress syndrome--oxygenation and outcome. Anaesth Intensive Care 27, 357-362

Dreyfuss D, Saumon G (1998): Ventilator-induced lung injury: lessons from experimental studies. Am J Respir Crit Care Med 157, 294-323

Engelmann L (2000): Das Open-lung-Konzept. Internist (Berl) 41, 985-994

Enger EL, O'Toole MF (1991): Noncardiogenic mechanisms of right heart dysfunction. J Cardiovasc Nurs $\underline{6}, 54-69$ 
Foex P (1991): Right ventricular function during ARDS. Acta Anaesthesiol Scand $\underline{95}$ Suppl, $72-79$

Fontes RB, Aguiar PH, Zanetti MV, Andrade F, Mandel M, Teixeira MJ (2003): Acute neurogenic pulmonary edema: case reports and literature review. J Neurosurg Anesthesiol $\underline{15}, 144-150$

Gattinoni L, Presenti A, Torresin A, Baglioni S, Rivolta M, Rossi F, Scarani F, Marcolin R, Cappelletti G (1986 a): Adult respiratory distress syndrome profiles by computed tomography. J Thorac Imaging $\underline{1}$, 25-30

Gattinoni L, Mascheroni D, Torresin A, Marcolin R, Fumagalli R, Vesconi S, Rossi GP, Rossi F, Baglioni S, Bassi F (1986 b): Morphological response to positive end expiratory pressure in acute respiratory failure. Computerized tomography study. Intensive Care Med $12,137-142$

Gattinoni L, Pesenti A, Avalli L, Rossi F, Bombino M (1987): Pressure-volume curve of total respiratory system in acute respiratory failure. Computed tomographic scan study. Am Rev Respir Dis 136, 730-736

Gattinoni L, Bombino M, Pelosi P, Lissoni A, Pesenti A, Fumagalli R, Tagliabue M (1994): Lung structure and function in different stages of severe adult respiratory distress syndrome. JAMA 271, 1772-1779

Gattinoni L, Caironi P, Cressoni M, Chiumello D, Ranieri VM, Quintel M, Russo S, Patroniti N, Cornejo R, Bugedo G (2006): Lung recruitment in patients with the acute respiratory distress syndrome. N Engl J Med $\underline{354}$, 1775-1786

Ghajar J (2000): Traumatic brain injury. Lancet 356, 923-929

Girgis K, Hamed H, Khater Y, Kacmarek RM (2006): A decremental PEEP trial identifies the PEEP level that maintains oxygenation after lung recruitment. Respir Care $\underline{51}$, 11321139

Goss CH, Brower RG, Hudson LD, Rubenfeld GD (2003): Incidence of acute lung injury in the United States. Crit Care Med 31, 1607-1611

Haitsma JJ, Papadakos PJ, Lachmann B (2004): Surfactant therapy for acute lung injury/acute respiratory distress syndrome. Curr Opin Crit Care 10, 18-22

Halperin BD, Feeley TW, Mihm FG, Chiles C, Guthaner DF, Blank NE (1985): Evaluation of the portable chest roentgenogram for quantitating extravascular lung water in critically ill adults. Chest $\underline{88}, 649-652$

Hardman JM, Manoukian A (2002): Pathology of head trauma. Neuroimaging Clin N Am $\underline{12}, 175-187$

Hedlund LW, Effmann EL, Bates WM, Beck JW, Goulding PL, Putman CE (1982): Pulmonary edema: a CT study of regional changes in lung density following oleic acid injury. J Comput Assist Tomogr 6, 939-946 
Hedlund LW, Vock P, Effmann EL, Putman CE (1985): Morphology of oleic acidinduced lung injury. Observations from computed tomography, specimen radiography, and histology. Invest Radiol 20, 2-8

Hickling KG, Walsh J, Henderson S, Jackson R (1994): Low mortality rate in adult respiratory distress syndrome using low-volume, pressure-limited ventilation with permissive hypercapnia: a prospective study. Crit Care Med ⒉, 1568-1578

Holland MC, Mackersie RC, Morabito D, Campbell AR, Kivett VA, Patel R, Erickson VR, Pittet JF (2003): The development of acute lung injury is associated with worse neurologic outcome in patients with severe traumatic brain injury. J Trauma $\underline{55}$, 106-111

Hounsfield GN (1973): Computerized transverse axial scanning (tomography). 1. Description of system. Br J Radiol 46, 1016-1022

Hutchinson PJ, O'Connell MT, Rothwell NJ, Hopkins SJ, Nortje J, Carpenter KL, Timofeev I, Al-Rawi PG, Menon DK, Pickard JD (2007): Inflammation in human brain injury: intracerebral concentrations of IL-1alpha, IL-1beta, and their endogenous inhibitor IL-1 ra. J Neurotrauma 24, 1545-1557

Jardin F, Gurdjian F, Delille F, Margairaz A (1979): Pulmonary hypertension in the adult respiratory distress syndrome (ARDS). Intensive Care Med $\underline{5}, 155-156$

Jardin F, Farcot JC, Boisante L, Curien N, Margairaz A, Bourdarias JP (1981): Influence of positive end-expiratory pressure on left ventricular performance. N Engl J Med $\underline{304}$, 387-392

Jardin F, Fellahi JL, Beauchet A, Vieillard-Baron A, Loubieres Y, Page B (1999): Improved prognosis of acute respiratory distress syndrome 15 years on. Intensive Care Med $\underline{25}, 936-941$

Kahn JM, Caldwell EC, Deem S, Newell DW, Heckbert SR, Rubenfeld GD (2006): Acute lung injury in patients with subarachnoid hemorrhage: incidence, risk factors, and outcome. Crit Care Med 34, 196-202

Kalsotra A, Zhao J, Anakk S, Dash PK, Strobel HW (2007): Brain trauma leads to enhanced lung inflammation and injury: evidence for role of P4504Fs in resolution. J Cereb Blood Flow Metab 27, 963-974

Kopp R, Kuhlen R, Max M (2003): Evidenzbasierte Medizin des akuten Lungenversagens. Anaesthesist $\underline{52}, 195-203$

Kopp R, Henzler D, Dembinski R, Kuhlen R (2004): Extrakorporale Membranoxygenierung beim akuten Lungenversagen. Anaesthesist $\underline{53}, 168-174$

Krishnan JA, Brower RG (2000): High-frequency ventilation for acute lung injury and ARDS. Chest 118, 795-807

Lachmann B (1992): Open up the lung and keep the lung open. Intensive Care Med $\underline{18}$, 319-321 
Lewis JF, Brackenbury A (2003): Role of exogenous surfactant in acute lung injury. Crit Care Med 31 Suppl, 324-328

Linden V, Palmer K, Reinhard J, Westman R, Ehren H, Granholm T, Frenckner B (2000): High survival in adult patients with acute respiratory distress syndrome treated by extracorporeal membrane oxygenation, minimal sedation, and pressure supported ventilation. Intensive Care Med 26, 1630-1637

Lohbrunner H, Deja M, Busch T, Spies CD, Rossaint R, Kaisers U (2004): Stellenwert von inhaliertem Stickstoffmonoxid bei der Behandlung des schweren akuten Lungenversagens. Anaesthesist $\underline{53}$, 771-782; quiz 783-774

Lopez-Aguilar J, Villagra A, Bernabe F, Murias G, Piacentini E, Real J, FernandezSegoviano P, Romero PV, Hotchkiss JR, Blanch L (2005): Massive brain injury enhances lung damage in an isolated lung model of ventilator-induced lung injury. Crit Care Med 33, 1077-1083

Lucas SM, Rothwell NJ, Gibson RM (2006): The role of inflammation in CNS injury and disease. Br J Pharmacol 147 Suppl 1, 232-240

Luecke T, Herrmann P, Quintel M (2000): Hochfrequenzoszillationsventilation (HFOV) bei akuter Lungenschädigung und ARDS. Anaesthesist $\underline{49}, 972-980$

Marini JJ (1996): Evolving concepts in the ventilatory management of acute respiratory distress syndrome. Clin Chest Med 17, 555-575

Mascia L (2009): Acute Lung Injury in Patients with Severe Brain Injury: A Double Hit Model. Neurocrit Care; elektronische Publikation am 24.06.2009

Mascia L, Zavala E, Bosma K, Pasero D, Decaroli D, Andrews P, Isnardi D, Davi A, Arguis MJ, Berardino M (2007): High tidal volume is associated with the development of acute lung injury after severe brain injury: an international observational study. Crit Care Med 35, 1815-1820

Mascia L, Sakr Y, Pasero D, Payen D, Reinhart K, Vincent JL (2008): Extracranial complications in patients with acute brain injury: a post-hoc analysis of the SOAP study. Intensive Care Med 34, 720-727

Maunder RJ, Shuman WP, McHugh JW, Marglin SI, Butler J (1986): Preservation of normal lung regions in the adult respiratory distress syndrome. Analysis by computed tomography. JAMA 255, 2463-2465

McClellan MD, Dauber IM, Weil JV (1989): Elevated intracranial pressure increases pulmonary vascular permeability to protein. J Appl Physiol 67, 1185-1191

McIntyre RW, Laws AK (1969): Positive expiratory pressure plateau: imporived gas exchange during mechanical ventilation. Can J Anaesth 16, 477 - 486

McKeating EG, Andrews PJ (1998): Cytokines and adhesion molecules in acute brain injury. Br J Anaesth $\underline{80}, 77-84$ 
Meade MO, Cook DJ, Guyatt GH, Slutsky AS, Arabi YM, Cooper DJ, Davies AR, Hand LE, Zhou Q, Thabane L (2008): Ventilation strategy using low tidal volumes, recruitment maneuvers, and high positive end-expiratory pressure for acute lung injury and acute respiratory distress syndrome: a randomized controlled trial. JAMA 299, 637-645

Meduri GU, Chinn A (1994): Fibroproliferation in late adult respiratory distress syndrome. Pathophysiology, clinical and laboratory manifestations, and response to corticosteroid rescue treatment. Chest $\underline{105}, 127-129$

Meduri GU, Chinn AJ, Leeper KV, Wunderink RG, Tolley E, Winer-Muram HT, Khare V, Eltorky M (1994): Corticosteroid rescue treatment of progressive fibroproliferation in late ARDS. Patterns of response and predictors of outcome. Chest $\underline{105}, 1516-1527$

Meduri GU, Headley AS, Golden E, Carson SJ, Umberger RA, Kelso T, Tolley EA (1998): Effect of prolonged methylprednisolone therapy in unresolving acute respiratory distress syndrome: a randomized controlled trial. JAMA 280, 159-165

Milberg JA, Davis DR, Steinberg KP, Hudson LD (1995): Improved survival of patients with acute respiratory distress syndrome (ARDS): 1983-1993. JAMA 273, 306-309

Moloney ED, Evans TW (2003): Pathophysiology and pharmacological treatment of pulmonary hypertension in acute respiratory distress syndrome. Eur Respir $\mathrm{J} 21,720$ 727

Mols G, Loop T, Geiger K, Farthmann E, Benzing A (2000): Extracorporeal membrane oxygenation: a ten-year experience. Am J Surg 180, 144-154

Montgomery AB, Stager MA, Carrico CJ, Hudson LD (1985): Causes of mortality in patients with the adult respiratory distress syndrome. Am Rev Respir Dis $\underline{132}, 485-489$

Mull RT (1984): Mass estimates by computed tomography: physical density from CT numbers. AJR Am J Roentgenol 143, 1101-1104

Narayan RK, Michel ME, Ansell B, Baethmann A, Biegon A, Bracken MB, Bullock MR, Choi SC, Clifton GL, Contant CF (2002): Clinical trials in head injury. J Neurotrauma 19, 503-557

Neumann P, Hedenstierna G (2001): Ventilation-perfusion distributions in different porcine lung injury models. Acta Anaesthesiol Scand $\underline{45}, 78-86$

NIH (2008): Traumatic brain injury: Hope through research. National Institute of Neurological Disorders and Stroke; Bethesda, Maryland http://www.ninds.nih.gov/disorders/tbi/detail tbi.htm

Ott L, McClain CJ, Gillespie M, Young B (1994): Cytokines and metabolic dysfunction after severe head injury. J Neurotrauma 11, 447-472

Park E, Bell JD, Baker AJ (2008): Traumatic brain injury: can the consequences be stopped? CMAJ $\underline{178}, 1163-1170$ 
Pelosi P, D'Andrea L, Vitale G, Pesenti A, Gattinoni L (1994): Vertical gradient of regional lung inflation in adult respiratory distress syndrome. Am J Respir Crit Care Med $\underline{149}, 8-13$

Piedalue F, Albert RK (2003): Prone positioning in acute respiratory distress syndrome. Respir Care Clin N Am $\underline{9}$, 495-509

Piek J, Chesnut RM, Marshall LF, van Berkum-Clark M, Klauber MR, Blunt BA, Eisenberg HM, Jane JA, Marmarou A, Foulkes MA (1992): Extracranial complications of severe head injury. J Neurosurg $\underline{77}, 901-907$

Pinsky MR (1990): The effects of mechanical ventilation on the cardiovascular system. Crit Care Clin $\underline{6}, 663-678$

Pulsion: PiCCO plus Zusatz-Monitor für Rechts- und Linksherzvolumina Bedienungsanleitung; hrsg. Pulsion Medical Systems AG, München, 2002, 5-6

Puneet P, Moochhala S, Bhatia M (2005): Chemokines in acute respiratory distress syndrome. Am J Physiol Lung Cell Mol Physiol 288, L3-15

Puybasset L, Cluzel P, Gusman P, Grenier P, Preteux F, Rouby JJ (2000 a): Regional distribution of gas and tissue in acute respiratory distress syndrome. I. Consequences for lung morphology. CT Scan ARDS Study Group. Intensive Care Med 26, 857-869

Puybasset L, Gusman P, Muller JC, Cluzel P, Coriat P, Rouby JJ (2000 b): Regional distribution of gas and tissue in acute respiratory distress syndrome. III. Consequences for the effects of positive end-expiratory pressure. CT Scan ARDS Study Group. Adult Respiratory Distress Syndrome. Intensive Care Med 26, 1215-1227

Rao V, Lyketsos C (2000): Neuropsychiatric sequelae of traumatic brain injury. Psychosomatics 41, 95-103

Rogers FB, Shackford SR, Trevisani GT, Davis JW, Mackersie RC, Hoyt DB (1995): Neurogenic pulmonary edema in fatal and nonfatal head injuries. J Trauma $\underline{39}$, 860-868

Rouby JJ, Puybasset L, Cluzel P, Richecoeur J, Lu Q, Grenier P (2000): Regional distribution of gas and tissue in acute respiratory distress syndrome. II. Physiological correlations and definition of an ARDS Severity Score. CT Scan ARDS Study Group. Intensive Care Med 26, 1046-1056

Rubenfeld GD, Caldwell E, Peabody E, Weaver J, Martin DP, Neff M, Stern EJ, Hudson LD (2005): Incidence and outcomes of acute lung injury. N Engl J Med 353, 1685-1693

Rylander C, Tylen U, Rossi-Norrlund R, Herrmann P, Quintel M, Bake B (2005): Uneven distribution of ventilation in acute respiratory distress syndrome. Crit Care $\underline{9}$, R165-171

Sakka SG, Ruhl CC, Pfeiffer UJ, Beale R, McLuckie A, Reinhart K, Meier-Hellmann A (2000): Assessment of cardiac preload and extravascular lung water by single transpulmonary thermodilution. Intensive Care Med 26, 180-187 
Sakka SG, Klein M, Reinhart K, Meier-Hellmann A (2002): Prognostic value of extravascular lung water in critically ill patients. Chest $\underline{122}, 2080-2086$

Salomone JP, Frame SB: Prehospital Care; in: Trauma; hrsg. v. Moore E, Feliciano D and Mattox K; McGraw-Hill, New-York, 2004, 117-118

Sauaia A, Moore FA, Moore EE, Moser KS, Brennan R, Read RA, Pons PT (1995): Epidemiology of trauma deaths: a reassessment. J Trauma 38, 185-193

Schneider GH, von Helden GH, Franke R, Lanksch WR, Unterberg A (1993): Influence of body position on jugular venous oxygen saturation, intracranial pressure and cerebral perfusion pressure. Acta Neurochir Suppl (Wien) 모, 107-112

Simon RP (1993): Neurogenic pulmonary edema. Neurol Clin 11, 309-323

Smith WS, Matthay MA (1997): Evidence for a hydrostatic mechanism in human neurogenic pulmonary edema. Chest 111, 1326-1333

Spragg RG, Lewis JF, Walmrath HD, Johannigman J, Bellingan G, Laterre PF, Witte MC, Richards GA, Rippin G, Rathgeb F (2004): Effect of recombinant surfactant protein $C$-based surfactant on the acute respiratory distress syndrome. N Engl J Med 351, 884892

Strieter RM, Kunkel SL (1994): Acute lung injury: the role of cytokines in the elicitation of neutrophils. J Investig Med 42, 640-651

The Acute Respiratory Distress Syndrome Network (2000): Ventilation with lower tidal volumes as compared with traditional tidal volumes for acute lung injury and the acute respiratory distress syndrome. N Engl J Med 342, 1301-1308

Theodore J, Robin ED (1976): Speculations on neurogenic pulmonary edema (NPE). Am Rev Respir Dis 113, 405-411

Touho H, Karasawa J, Shishido H, Yamada K, Yamazaki Y (1989): Neurogenic pulmonary edema in the acute stage of hemorrhagic cerebrovascular disease. Neurosurgery $\underline{25}, 762-768$

Urdaneta F, Layon AJ (2003): Respiratory complications in patients with traumatic cervical spine injuries: case report and review of the literature. J Clin Anesth 15, 398405

Valadka A: Injury to the cranium; in: Trauma; hrsg. v. Moore E, Feliciano D and Mattox K; McGraw-Hill, New York, 2004, 385-406

Valenza F, Guglielmi M, Maffioletti M, Tedesco C, Maccagni P, Fossali T, Aletti G, Porro GA, Irace M, Carlesso E (2005): Prone position delays the progression of ventilatorinduced lung injury in rats: does lung strain distribution play a role? Crit Care Med $\underline{33}$, 361-367 
Verweij BH, Muizelaar JP, Vinas FC, Peterson PL, Xiong Y, Lee CP (2000): Impaired cerebral mitochondrial function after traumatic brain injury in humans. J Neurosurg $\underline{93}$, 815-820

Vieillard-Baron A, Schmitt JM, Augarde R, Fellahi JL, Prin S, Page B, Beauchet A, Jardin $F$ (2001): Acute cor pulmonale in acute respiratory distress syndrome submitted to protective ventilation: incidence, clinical implications, and prognosis. Crit Care Med $\underline{29}, 1551-1555$

Vieira SR, Puybasset L, Richecoeur J, Lu Q, Cluzel P, Gusman PB, Coriat P, Rouby JJ (1998): A lung computed tomographic assessment of positive end-expiratory pressureinduced lung overdistension. Am J Respir Crit Care Med 158, 1571-1577

Walmrath D, Olschewski H, Grimminger F, Seeger W (1997): NO und alternative inhalative Therapieansätze bei pulmonaler Hypertonie. Internist (Berl) $\underline{38}$, 453-460

Wartenberg KE, Schmidt JM, Claassen J, Temes RE, Frontera JA, Ostapkovich N, Parra A, Connolly ES, Mayer SA (2006): Impact of medical complications on outcome after subarachnoid hemorrhage. Crit Care Med $\underline{34}$, 617-623; quiz 624

Weber-Carstensen S, Lohbrunner H (1999): Lungenprotektive Strategien zur Therapie des ARDS. Anasthesiol Intensivmed Notfallmed Schmerzther 36, 677-693

Witko-Sarsat V, Rieu P, Descamps-Latscha B, Lesavre P, Halbwachs-Mecarelli L (2000): Neutrophils: molecules, functions and pathophysiological aspects. Lab Invest $\underline{80}, 617-653$

Zink BJ (2001): Traumatic brain injury outcome: concepts for emergency care. Ann Emerg Med 37, 318-332

Zwissler B (2002): Inhalierte Vasodilatatoren. Anaesthesist 51, 603-624

Zygun DA, Kortbeek JB, Fick GH, Laupland KB, Doig CJ (2005): Non-neurologic organ dysfunction in severe traumatic brain injury. Crit Care Med $\underline{33}$, 654-660 


\section{Amendment}

Zur Sicherung guter wissenschaftlicher Praxis weisen der Vefasser dieser Dissertationsschrift, Herr Philip Sauter und der Doktorvater Prof. M. Quintel daraufhin, dass ein bestimmter und durch Hinweise in den Legenden genau bestimmbarer Anteil dieser Arbeit bereits publiziert wurde.

Der Name des Doktoranden (Philip Sauter) wurde allein durch ein Versäumnis des Seniorautors (Prof. M. Quintel) nicht in der Publikation (Intensive Care Med (2011) 37:1182-1191: Acute effects of intracranial hypertension and ARDS on pulmonary and neuronal damage: a randomized experimental study in pigs) aufgeführt.

Die Promotionskomission wurde hiervon in Kenntnis gesetzt. Die Komission hat am 10.11.2011 festgestellt, dass Herr Philip Sauter glaubhaft dokumentieren kann, einen signifikanten Beitrag an der Datenerstellung und -auswertung geleistet zu haben und dass ihm nach anerkannten Grundsätzen der Autorenregelung eine Koautorenschaft auf besagter Publikation zustünde.

Die Arbeit wurde am 10.11.2011 endgültig als Dissertationsleistung angenommen.

Prof. M. Quintel

Philip Sauter 